\title{
Density and excess volume for four systems involving eugenol and furan
}

\author{
Christophe Coquelet*, Eric Auger, Alain Valtz
}

Mines ParisTech PSL University, CTP-Centre of Thermodynamics of Processes, 35 Rue Saint Honoré, 77305 Fontainebleau, France.

\begin{abstract}
Density and speed of sound measurements have been performed, at atmospheric pressure, using an Anton Paar digital vibrating tube densitometer for pure ethanol, 1-Octanol, nhexane, furan and eugenol, from 278.15 to $323.15 \mathrm{~K}$ and for binary mixtures of furan + ethanol, furan + 1-Octanol, eugenol + 1-Octanol and eugenol + n-hexane from 278.15 to $323.15 \mathrm{~K}$. Excess molar volumes were calculated and compared. The Redlich Kister correlation was used to correlate the data. In order to identify the most relevant molecular interaction which contribute to the excess molar volume, the Prigogine Flory Patterson theory was applied to correlate and predict the excess molar volume of the mixtures.
\end{abstract}

Keywords: Excess molar volume, Partial molar volume

*: Corresponding author, Email: Christophe.coquelet@mines-paristech.fr Tel.: + (33) 1646949 62. Fax: + (33) 1646949 68. 


\section{Introduction}

Accurate predictions of the thermodynamic properties of multi-component fluid mixtures are essential for both the optimization of current industrial processes and the design of new ones. Biomass is considered as the new source of raw material to produce new molecules and also energy (biofuels, biogas). In several operation, units like heat exchangers, liquid-liquid extraction and distillation columns require a good estimation of density, speed of sound and so excess volume for the mixtures concerned to design them. Among these systems, the second-generation biofuels or mixture of oxygenated compounds involve several molecules and mixtures whose such properties are unknown. Mixing of oxygenated compounds generally leads to solutions that do not behave ideally. The deviation from ideal behavior can be conveniently expressed by excess properties.

Excess properties are also very useful to understand the mixing state in terms of intermolecular interactions. The information concerning molecular interaction are required to the design of solvents for industrial applications. Solvents are designed to extract molecule of interest and to let the nondesirable molecules in the original media.

In this article, we will focus on two chemicals of interest furan and eugenol which are used as intermediate in chemistry or in medicine. Indeed, they are widely used in the production of the solvent tetrahydrofuran, as additives for detergents and herbicides, in dentistry applications, as antiseptic and anesthetic products in pharmacy and in aquaculture [1],[2]. Ethanol, 1-Octanol and nhexane are the studied solvents. Some volumetric properties of the systems Eugenol+Hexan, Eugenol+1-Octanol, Furan+Ethanol and Furan+1-Octanol are also presented.

First, new experimental measurements of density and excess volume for the binary mixtures Eugenol+n-hexan, Eugenol+1-Octanol, Furan+Ethanol and Furan+1-Octanol are presented. Then, these new experimental excess volumes are correlated by the Redlich-Kister correlation. Finally, the Prigogine-Flory-Patterson (PFP) Theory is applied to identify the most predominant molecular interaction.

\section{Experimental details}

\subsection{Materials purities and suppliers}

Table 1 presents the chemical species, suppliers, purity and refractive index. Compositions are prepared gravimetrically. 
Table 1: Chemical sample

\begin{tabular}{lcccccc}
\hline \multicolumn{1}{c}{ Compound } & $\begin{array}{c}\text { CAS } \\
\text { Number }\end{array}$ & Formula & Supplier & $\begin{array}{c}\text { Purity } \\
(\mathrm{GC})\end{array}$ & $\begin{array}{c}\text { Refractive } \\
\text { index }\end{array}$ & $\begin{array}{c}\text { nD } \\
\text { litt }\end{array}$ \\
& & & & & $(293.15 \mathrm{~K})$ & \\
\hline Furane (stabilized) & $110-00-9$ & $\mathrm{C}_{4} \mathrm{H}_{4} \mathrm{O}$ & SIGMA-ALDRICH & $>99 \%$ & $1.4215_{6}$ & $1.41871^{\mathrm{a}}$ \\
n-Hexane & $110-54-3$ & $\mathrm{C}_{6} \mathrm{H}_{14}$ & SIGMA-ALDRICH & $>99 \%$ & $1.3754_{2}$ & $1.37226^{\mathrm{a}}$ \\
Eugenol & $97-53-0$ & $\mathrm{C}_{10} \mathrm{H}_{12} \mathrm{O}_{2}$ & ACROS & $>99 \%$ & $1.5408_{8}$ & $1.539^{\mathrm{b}}$ \\
1-1-Octanol & $111-87-5$ & $\mathrm{C}_{8} \mathrm{H}_{18} \mathrm{O}$ & SIGMA-ALDRICH & $>99 \%$ & $1.4292_{1}$ & $1.4276^{\mathrm{a}}$ \\
Ethanol & $64-17-5$ & $\mathrm{C}_{2} \mathrm{H}_{6} \mathrm{O}$ & FLUKA & $>99.8 \%$ & $1.3614_{8}$ & $1.35941^{\mathrm{a}}$ \\
\hline
\end{tabular}

*: Apparatus; Anton Paar ABBEMAT 300 acuracy +/- 0.0001, (a): Value from Component Plus (Prosim, France), (b): given by Gladstone [3] at 290.33 K, GC: Gas Chromatograph

\subsection{Experimental method}

Density measurements have been all performed with the DSA5000M Anton Paar digital vibrating tube densimeter. The oscillating period or frequency, measured by the densitometer, is depending on the tube mass and therefore on the fluid density. Eq. 1 is used for relating the period of vibration, $\tau$, to density, $\rho$ :

$\rho=a+b \tau^{2}$

where $a$ and $b$ are constants to be adjusted. For these purposes we have used bi-distilled and degassed water, and dry air, at $293.15 \mathrm{~K}$. Uncertainty on measured density is estimated lower than $10^{-5} \mathrm{~g} . \mathrm{cm}^{-3}$. One platinum resistance thermometer with $0.01 \mathrm{~K}$ accuracy is used for temperature measurements. The sample densities are then measured at thermal equilibrium for various temperatures. This densitometer permits also the measurement of speed of sound with an accuracy of $0.5 \mathrm{~m} \cdot \mathrm{s}^{-1}$.

To prepare the mixtures, an empty $20 \mathrm{~cm}^{3}$ glass bottle is air-tight closed with a septum and then put under vacuum using a vacuum pump where a needle is introduced through the septum. Empty bottle is weighed, and then the less volatile component, freshly degassed, is introduced by means of a syringe. After weighing the bottle loaded with the first component, the more volatile one is added 
similarly and then the bottle is weighed again. All weighing are performed using an analytical balance with $10^{-4} \mathrm{~g}$ accuracy, correspondingly the uncertainty is estimated to be lower than $2 \times 10^{-5}$ for mole fractions. Maximum uncertainty resulting in the calculation of $v^{\mathrm{E}}$ is estimated to be less than $u_{v^{E}}=0.003 \mathrm{~cm}^{3} \cdot \mathrm{mol}^{-1}$ using Eqs. 2-4. Atmospheric pressure is measured by a GE Druck DPI 142 Barometric Indicator with an uncertainty $u_{p}=0.029 \mathrm{kPa}$.

$u_{\rho m}=\sqrt{u_{\rho}^{2}+u_{x}^{2}}$

$u_{x 1}=x_{1} x_{2} u_{m} \sqrt{\frac{1}{\left(m_{1}\right)^{2}}+\frac{1}{\left(m_{2}\right)^{2}}}$

$u_{v^{E}}=\sqrt{\left(\left(\frac{x_{1} M_{1}+x_{2} M_{2}}{\rho_{m}^{2}}\right)^{2}+\left(\frac{x_{1} M_{1}}{\rho_{1}^{2}}\right)^{2}+\left(\frac{x_{2} M_{2}}{\rho_{2}^{2}}\right)^{2}\right) u_{\rho m}^{2}+\left(\frac{M_{1}+M_{2}}{\rho_{m}}-\frac{M_{1}}{\rho_{1}}-\frac{M_{2}}{\rho_{2}}\right)^{2} u_{x}^{2}}$

With $M_{i}$ the molar mass of component $i, m_{i}$ the mass of component $i, \rho_{i}$ the density of component $i$, $\rho_{\mathrm{m}}$ density of the mixture.

\section{Results and Discussions}

The density values of pure chemicals measured using the DSA 5000M Anton Paar densitometer are presented as a function of temperature in Table 2. Comparison with literature was done and presented in Supplementary Information. These data will be also considered to determinate the pure component parameters for the data treatment using PFP model (see section 3.2). 
Table 2: Density of each pure component studied at atmospheric pressure $\left(U_{p}= \pm 0.03 \mathrm{kPa}, U_{T}= \pm 0.01 \mathrm{~K}, U_{\rho}= \pm 10^{-5} \mathrm{~g} . \mathrm{cm}^{-3}(\mathrm{k}=2)\right)$.

\begin{tabular}{|c|c|c|c|c|c|c|c|c|c|}
\hline \multicolumn{2}{|c|}{ Eugenol } & \multicolumn{2}{|c|}{ Furan } & \multicolumn{2}{|c|}{ Ethanol } & \multicolumn{2}{|c|}{ 1-Octanol } & \multicolumn{2}{|c|}{ n-hexane } \\
\hline$T / \mathrm{K}$ & $\rho_{\exp } /$ g.cm $^{-3}$ & $T / \mathrm{K}$ & $\rho_{\text {exp }} /$ g.cm $^{-3}$ & $T / \mathrm{K}$ & $\rho_{\exp } /$ g.cm ${ }^{-3}$ & $T / \mathrm{K}$ & $\rho_{\mathrm{exp}} / \mathbf{g . c m}^{-3}$ & $T / \mathrm{K}$ & $\rho_{\exp } / \mathbf{g . c m}^{-3}$ \\
\hline 273.15 & 1.08383 & 278.16 & 0.95856 & 278.16 & 0.80214 & 278.16 & 0.83536 & 283.15 & 0.66887 \\
\hline 277.16 & 1.08028 & 280.15 & 0.95589 & 280.14 & 0.80046 & 280.15 & 0.83400 & 293.14 & 0.65992 \\
\hline 279.15 & 1.07850 & 281.15 & 0.95455 & 281.14 & 0.79962 & 281.15 & 0.83332 & 298.14 & 0.65538 \\
\hline 285.15 & 1.07320 & 284.15 & 0.95050 & 284.14 & 0.79707 & 284.15 & 0.83126 & 313.15 & 0.64157 \\
\hline 287.15 & 1.07143 & 285.14 & 0.94916 & 285.14 & 0.79622 & 285.15 & 0.83058 & 318.15 & 0.63687 \\
\hline 289.15 & 1.06967 & 286.14 & 0.94780 & 286.14 & 0.79537 & 286.15 & 0.82990 & 323.14 & 0.63212 \\
\hline 291.15 & 1.06791 & 287.14 & 0.94644 & 287.15 & 0.79451 & 287.15 & 0.82921 & & \\
\hline 293.15 & 1.06614 & 288.14 & 0.94508 & 288.15 & 0.79366 & 288.15 & 0.82853 & & \\
\hline 303.16 & 1.05730 & 293.14 & 0.93824 & 293.15 & 0.78939 & 293.15 & 0.82509 & & \\
\hline 305.16 & 1.05553 & 294.15 & 0.93687 & 294.15 & 0.78854 & 294.15 & 0.82440 & & \\
\hline 307.16 & 1.05376 & 295.15 & 0.93549 & 295.15 & 0.78768 & 295.15 & 0.82371 & & \\
\hline 309.16 & 1.05199 & 296.15 & 0.93410 & 296.15 & 0.78682 & 296.15 & 0.82302 & & \\
\hline 311.16 & 1.05022 & 297.15 & 0.93272 & 297.15 & 0.78596 & 297.15 & 0.82233 & & \\
\hline 313.16 & 1.04845 & 298.15 & 0.93133 & 298.15 & 0.78510 & 298.15 & 0.82164 & & \\
\hline 315.16 & 1.04668 & 299.15 & 0.92995 & 299.15 & 0.78424 & 299.15 & 0.82095 & & \\
\hline 317.16 & 1.04492 & 300.15 & 0.92856 & 300.15 & 0.78338 & 300.15 & 0.82025 & & \\
\hline 319.16 & 1.04315 & 301.15 & 0.92716 & 301.15 & 0.78251 & 301.15 & 0.81956 & & \\
\hline 321.16 & 1.04138 & 302.15 & 0.92576 & 302.15 & 0.78165 & 302.15 & 0.81886 & & \\
\hline
\end{tabular}




\begin{tabular}{llllllll}
325.16 & 1.03784 & 304.15 & 0.92296 & 304.15 & 0.77991 & 304.15 & 0.81747 \\
327.16 & 1.03607 & & & & & & \\
329.16 & 1.03430 & & & & & & \\
331.16 & 1.03253 & & & & & & \\
335.15 & 1.02898 & & & & & \\
337.15 & 1.02720 & & & & & \\
339.15 & 1.02542 & & & & & \\
341.15 & 1.02365 & & & & \\
343.15 & 1.02187 & & & \\
\hline
\end{tabular}


These data were used to calculate the excess volume. The excess molar volume $v^{E}$, is calculated using Eq. 5.

$v^{E}=v-x_{1} v_{1}^{*}-x_{2} v_{2}^{*}$

where $x_{1}$ and $x_{2}$ represent mole fractions and $v_{1}^{*}$ and $v_{2}^{*}$ are the molar volumes of components 1 and 2 respectively. $v$ stands for the molar volume of mixture. Using the measured density $\rho$, Eq. 5 can be rewritten as:

$v^{E}=\left[\frac{x_{1} M_{1}+x_{2} M_{2}}{\rho}\right]-\frac{x_{1} M_{1}}{\rho_{1}^{*}}-\frac{x_{2} M_{2}}{\rho_{2}^{*}}$

where $M_{1}$ and $M_{2}$ are the molar masses $\rho_{1}^{*}$ and $\rho_{2}^{*}$ are the densities of components (1) and (2) respectively. $\rho$ stands for the density of the mixture.

\subsection{Redlich Kister data treatment}

Usually Redlich-Kister (RK) correlation [4] is chosen to correlate excess molar volume of binary systems. Eq. 6 presents the correlation.

$v^{E}=x_{1} x_{2} \sum_{i} A_{i}\left(x_{1}-x_{2}\right)^{i}$

The coefficients $\left(A_{i}\right)$ have to be determined. The variance $\sigma$, corresponding to each fit, is calculated using Eq. 7.

$\sigma=\sqrt{\left[\sum \frac{\left(v^{E}-v_{c a l}^{E}\right)^{2}}{N_{\exp }-P}\right]}$

where $P$ is the number of parameters $\left(A_{n}\right)$ and $N_{\text {exp }}$ represents number of experimental data. 
The main difficulty of the RK treatment is the selection of the number of parameter $A_{i}$. In order to do that, it is recommended to investigate $\frac{v^{E}}{x_{1} x_{2}}$ as a function of molar composition $\mathrm{x}_{1}$. This quantity gives us useful information concerning volumetric properties, particularly at low concentration as suggested by Desnoyers and Perron [5]. This term is directly related to the apparent molar volume and so can be assimilated to a thermodynamic property. Eq. 8 shows that $\frac{v^{E}}{x_{1} x_{2}}$ is directly linked to the apparent molar volume.

$$
\frac{v^{E}}{x_{1} x_{2}}=\frac{v_{2, \varphi}-v_{2}^{*}}{x_{1}}=\frac{v_{1, \varphi}-v_{1}^{*}}{x_{2}}
$$

where $v_{i, \varphi}$ is the apparent molar volume. Desnoyers and Perron indicates that the change of the $\frac{v^{E}}{x_{1} x_{2}}$ slope can be attributed to various factor: first one, the size and shape of molecules, the second one the intermolecular interaction energy differences at dilution and the third one the formation of chemical complex containing unlike molecules. Also, at infinite dilution, $\frac{v^{E}}{x_{1} x_{2}}$ decreases like apparent molar volume. There are two contributions for the apparent molar volume of a molecule: the volume of the molecule and the free volume space. By analysis the evolution of $\frac{v^{E}}{x_{1} x_{2}}$ as a function of $\mathrm{x}_{1}$ we have selected the number of RK parameters for all the investigated binary systems.

The partial molar volume $\overline{v_{i}}\left(\mathrm{~cm}^{3} \cdot \mathrm{mol}^{-1}\right)$ of each component $\mathrm{i}$ has been calculated using Eq. 9, with $V$, the volume of the mixture $\left(\mathrm{cm}^{3}\right)$.

$$
\overline{v_{i}}=\left(\frac{\partial V}{\partial n_{i}}\right)_{T, P, n_{j}}
$$

By differentiating Eq. 6 with respect to $n_{i}$ and combining the result to Eq. 9 leads to equations for the partial molar volumes of the different species (Eqs. 10 and 11). 
$\overline{v_{1}}=v^{E}+v_{1}^{*}-x_{2}\left(\frac{\partial v^{E}}{\partial x_{2}}\right)_{T, P}$

$$
\overline{v_{2}}=v^{E}+v_{2}^{*}-x_{1}\left(\frac{\partial v^{E}}{\partial x_{1}}\right)_{T, P}
$$

Using Redlich Kister equation, we can obtain the expression of partial molar volumes (Eqs. 12 and 13) with respect to $x_{i}$.

$$
\begin{aligned}
& \overline{v_{1}}=v_{1}^{*}+x_{2}^{2} \sum A_{n}\left(1-2 x_{2}\right)^{n}+2 x_{2}^{2}\left(1-x_{2}\right) \sum n A_{n}\left(1-2 x_{2}\right)^{n-1} \\
& \overline{v_{2}}=v_{2}^{*}+\left(1-x_{2}\right)^{2} \sum A_{n}\left(1-2 x_{2}\right)^{n}-2 x_{2}\left(1-x_{2}\right)^{2} \sum n A_{n}\left(1-2 x_{2}\right)^{n-1}
\end{aligned}
$$

\section{a. Furan ethanol binary system}

Table 3 presents the values of mixtures densities and excess volume. Table 4 presents our results. Following Desnoyers and Perron's method we have considered 4 Redlich Kister parameters. Figs. 1-2 presents respectively, $\frac{v^{E}}{x_{1} x_{2}}$ as a function of $\mathrm{x}_{1}$ and $\mathrm{v}^{\mathrm{E}}$ as a function of $\mathrm{x}_{1}$, at 2 different temperatures. As we can see, excess molar volume is negative but becomes positive close to pure ethanol. The analysis of apparent molar volume does not reveal any original behavior. But we can see that for furan at infinite dilution, apparent molar volume decreases certainly due to the fact there are more $\mathrm{H}$ bonds between ethanol molecules who surround the furan molecule. Excess molar volume is positive when we approach pure furan and when we increase the temperature. There is less $\mathrm{H}$-bond between the two chemicals and so as there is an importance difference between the two dipole moments (1.69D for ethanol and 0.66D for furan [6]), there is less cross interaction and so an increasing of excess volume. 
Table 3: Densities $(\rho)$ and excess molar volumes $\left(v^{E}\right)$ for furan $(1)+$ ethanol $(2)$ binary system as a function of furan mole fraction at atmospheric pressure $\left(U_{p}= \pm 0.03\right.$ $\left.\mathrm{kPa}, \mathrm{U}_{\mathrm{x} 1}= \pm 2 \times 10^{-5}, \mathrm{U}_{\mathrm{T}}= \pm 0.01 \mathrm{~K}, \mathrm{U}_{\mathrm{\rho}}= \pm 10^{-5} \mathrm{~g} \cdot \mathrm{cm}^{-3}, \mathrm{U}_{\mathrm{VE}}= \pm 0.003 \mathrm{~cm}^{3} \cdot \mathrm{mol}^{-1}(\mathrm{k}=2)\right)$.

\begin{tabular}{|c|c|c|c|c|c|c|c|c|c|c|c|c|}
\hline$x_{1}$ & $d / \mathrm{g} . \mathrm{cm}^{-3}$ & $\begin{array}{c}V^{E} \\
/ \mathrm{cm}^{3} \cdot \mathrm{mol}^{-1}\end{array}$ & $d / \mathrm{g} . \mathrm{cm}^{-3}$ & $\begin{array}{c}V^{E} \\
/ \mathrm{cm}^{3} \cdot \mathrm{mol}^{-1}\end{array}$ & $d / \mathrm{g} . \mathrm{cm}^{-3}$ & $\begin{array}{c}V^{E} \\
/ \mathrm{cm}^{3} \cdot \mathrm{mol}^{-1}\end{array}$ & $d / \mathrm{g} . \mathrm{cm}^{-3}$ & $\begin{array}{c}V^{E} \\
/ \mathrm{cm}^{3} \cdot \mathrm{mol}^{-}\end{array}$ & $d / \mathrm{g} . \mathrm{cm}^{-1}$ & $\begin{array}{c}V^{E} \\
/ \mathrm{cm}^{3} \cdot \mathrm{mol}^{-}\end{array}$ & $\begin{array}{c}d \\
\text { /g.cm }{ }^{-3}\end{array}$ & $\begin{array}{c}V^{E} \\
/ \mathrm{cm}^{3} \cdot \mathrm{mol}\end{array}$ \\
\hline & \multicolumn{2}{|c|}{$\mathrm{T}=278.15 \mathrm{~K}$} & \multicolumn{2}{|c|}{$\mathrm{T}=283.15 \mathrm{~K}$} & \multicolumn{2}{|c|}{$\mathrm{T}=288.15 \mathrm{~K}$} & \multicolumn{2}{|c|}{$\mathrm{T}=293.15 \mathrm{~K}$} & \multicolumn{2}{|c|}{$\mathrm{T}=298.15 \mathrm{~K}$} & \multicolumn{2}{|c|}{$\mathrm{T}=303.15 \mathrm{~K}$} \\
\hline 0.0248 & 0.8075 & -0.045 & 0.80322 & -0.044 & 0.79889 & -0.044 & 0.79454 & -0.044 & 0.79018 & -0.045 & 0.7858 & -0.044 \\
\hline 0.0501 & 0.8128 & -0.075 & 0.80839 & -0.074 & 0.80398 & -0.074 & 0.79956 & -0.074 & 0.79507 & -0.071 & 0.7906 & -0.069 \\
\hline 0.0748 & 0.8177 & -0.095 & 0.81321 & -0.092 & 0.80871 & -0.091 & 0.80420 & -0.090 & 0.79967 & -0.089 & 0.7951 & -0.085 \\
\hline 0.1005 & 0.8227 & -0.113 & 0.81817 & -0.110 & 0.81359 & -0.108 & 0.80900 & -0.107 & 0.80437 & -0.104 & 0.7997 & -0.099 \\
\hline 0.1505 & 0.8323 & -0.149 & 0.82763 & -0.145 & 0.82289 & -0.141 & 0.81812 & -0.137 & 0.81333 & -0.134 & 0.8085 & -0.130 \\
\hline 0.2027 & 0.8419 & -0.169 & 0.83705 & -0.164 & 0.83215 & -0.159 & 0.82723 & -0.155 & 0.82227 & -0.150 & 0.8172 & -0.140 \\
\hline 0.2549 & 0.8512 & -0.186 & 0.84622 & -0.180 & 0.84116 & -0.174 & 0.83607 & -0.168 & 0.83093 & -0.160 & 0.8257 & -0.151 \\
\hline 0.2999 & 0.8590 & -0.194 & 0.85386 & -0.187 & 0.84867 & -0.180 & 0.84343 & -0.172 & 0.83820 & -0.167 & 0.8328 & -0.154 \\
\hline 0.3526 & 0.8678 & -0.194 & 0.86251 & -0.187 & 0.85716 & -0.179 & 0.85178 & -0.170 & 0.84634 & -0.160 & 0.8408 & -0.149 \\
\hline 0.3999 & 0.8754 & -0.185 & 0.86996 & -0.177 & 0.86449 & -0.169 & 0.85897 & -0.159 & 0.85339 & -0.148 & 0.8478 & -0.135 \\
\hline 0.4500 & 0.8833 & -0.177 & 0.8777 & -0.168 & 0.87209 & -0.158 & 0.86643 & -0.148 & 0.86079 & -0.142 & 0.8549 & -0.123 \\
\hline 0.4998 & 0.8909 & -0.163 & 0.88518 & -0.155 & 0.87943 & -0.145 & 0.87364 & -0.134 & 0.86780 & -0.123 & 0.8619 & -0.108 \\
\hline 0.4998 & 0.8909 & -0.167 & 0.88523 & -0.158 & 0.87949 & -0.148 & 0.87369 & -0.137 & 0.86785 & -0.126 & 0.8619 & -0.111 \\
\hline 0.5499 & 0.8983 & -0.148 & 0.89251 & -0.139 & 0.88664 & -0.129 & 0.88071 & -0.117 & 0.87474 & -0.106 & 0.8687 & -0.090 \\
\hline 0.6000 & 0.9055 & -0.128 & 0.8996 & -0.118 & 0.89361 & -0.108 & 0.88756 & -0.096 & 0.88145 & -0.084 & 0.8753 & -0.068 \\
\hline 0.6500 & 0.9126 & -0.111 & 0.90659 & -0.102 & 0.90047 & -0.090 & 0.89430 & -0.079 & 0.88807 & -0.067 & 0.8818 & -0.051 \\
\hline 0.7009 & 0.9197 & -0.090 & 0.91349 & -0.080 & 0.90726 & -0.069 & 0.90097 & -0.058 & 0.89462 & -0.046 & 0.8882 & -0.032 \\
\hline 0.7500 & 0.9263 & -0.069 & 0.92003 & -0.060 & 0.91369 & -0.050 & 0.90729 & -0.039 & 0.90082 & -0.028 & 0.8943 & -0.017 \\
\hline 0.8000 & 0.9329 & -0.050 & 0.92657 & -0.042 & 0.92012 & -0.033 & 0.91362 & -0.023 & 0.90705 & -0.013 & 0.9004 & 0.001 \\
\hline 0.8499 & 0.9394 & -0.031 & 0.93296 & -0.024 & 0.92642 & -0.016 & 0.91981 & -0.007 & 0.91314 & 0.002 & 0.9064 & 0.014 \\
\hline 0.9000 & 0.9459 & -0.014 & 0.93929 & -0.008 & 0.93266 & -0.002 & 0.92596 & 0.005 & 0.91919 & 0.013 & 0.9124 & 0.021 \\
\hline 0.9250 & 0.9491 & -0.009 & 0.94245 & -0.004 & 0.93577 & 0.002 & 0.92903 & 0.007 & 0.92221 & 0.014 & 0.9153 & 0.023 \\
\hline 0.9500 & 0.9523 & -0.007 & 0.94562 & -0.003 & 0.93890 & 0.001 & 0.93211 & 0.006 & 0.92527 & 0.010 & 0.9183 & 0.017 \\
\hline 0.9750 & 0.9554 & -0.002 & 0.94874 & -0.001 & 0.94198 & 0.003 & 0.93517 & 0.005 & 0.92830 & 0.006 & 0.9213 & 0.012 \\
\hline
\end{tabular}


Table 4: Redlich-Kister parameters and deviation for furan + ethanol binary system.

\begin{tabular}{|c|c|c|c|c|c|}
\hline \multirow{2}{*}{$\mathbf{T} / \mathbf{K}$} & \multicolumn{4}{|c|}{ Redlich Kister parameters } & \multirow[t]{2}{*}{$\begin{array}{c}\text { Variance } \\
\sigma, \text { equation } 7\end{array}$} \\
\hline & $\mathbf{A}_{\mathbf{0}}$ & $\mathbf{A}_{1}$ & $\mathbf{A}_{2}$ & $\mathbf{A}_{\mathbf{3}}$ & \\
\hline 278.15 & -0.654 & 0.565 & -0.114 & 0.223 & 0.003 \\
\hline 283.15 & -0.617 & 0.578 & -0.111 & 0.236 & 0.003 \\
\hline 288.15 & -0.578 & 0.595 & -0.098 & 0.269 & 0.004 \\
\hline 293.15 & -0.534 & 0.610 & -0.090 & 0.303 & 0.004 \\
\hline 298.15 & -0.492 & 0.637 & -0.070 & 0.312 & 0.004 \\
\hline 303.15 & -0.431 & 0.639 & -0.056 & 0.382 & 0.004 \\
\hline
\end{tabular}

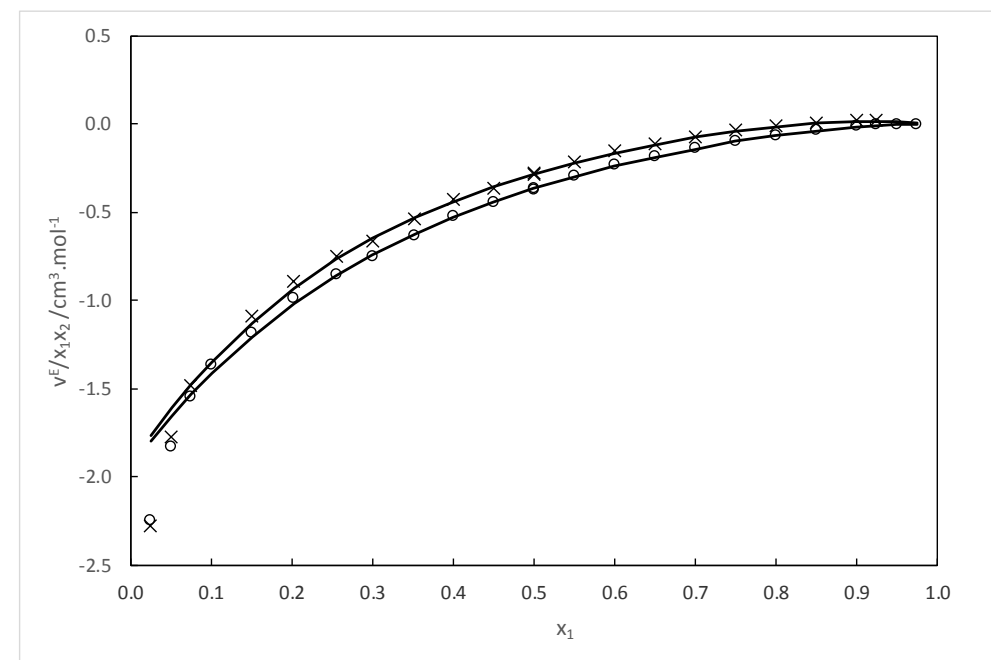

Figure 1: $\frac{v^{E}}{x_{1} x_{2}}$ for furan(1) + ethanol(2) system as a function of furan mole fraction at atmospheric pressure and 2 different temperatures: (0) $283.15 \mathrm{~K},(x) 303.15 \mathrm{~K}$, solid line: Redlich Kister correlation. 


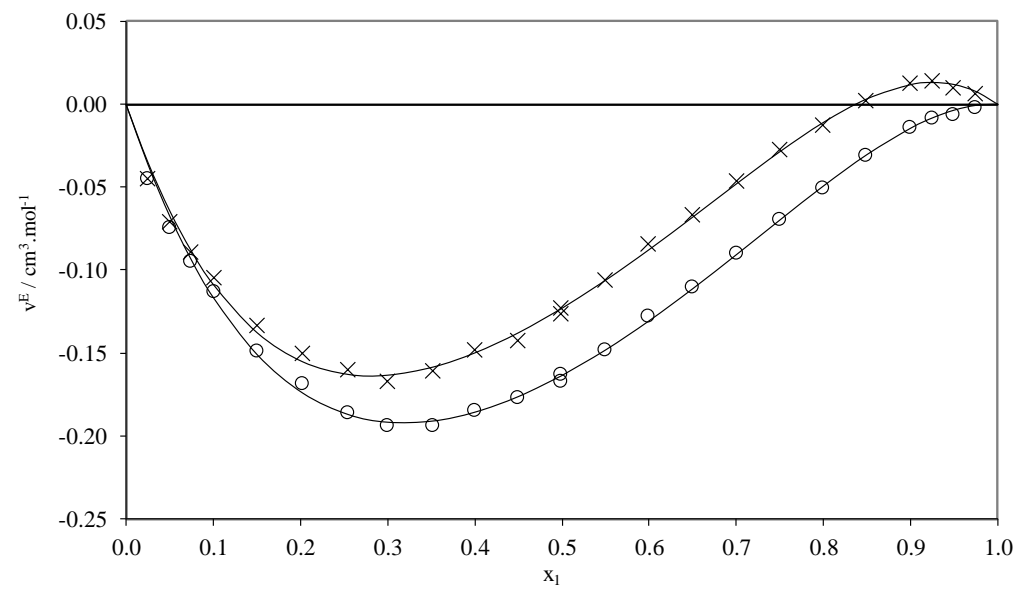

Figure 2: Excess molar volume $\left(v^{E}\right)$ for furan(1) + ethanol(2) binary system as a function of composition at atmospheric pressure and 2 different temperatures: (o) $283.15 \mathrm{~K},(x) 303.15 \mathrm{~K}$, solid line: Redlich Kister correlation.

\section{b. Furan 1-Octanol binary system}

Table 5 presents the values of mixtures densities and excess volume. Table 6 presents our results. Following Desnoyers and Perron's method we have considered 3 RK parameters. Figs 3-4 presents $\frac{v^{E}}{x_{1} x_{2}}$ as a function of $\mathrm{x}_{1}$ and $\mathrm{v}^{\mathrm{E}}$ as a function of $\mathrm{x}_{1}$ at 2 different temperatures. We can observe that there is no specific behavior. We can consider that it is a mixture of two liquids with similar size. As we can see, excess molar volume is positive, it is certainly due the difference of polarity between the two chemicals (furan: $0.66 \mathrm{D}$ and 1-Octanol: $1.66 \mathrm{D}$ [6]). In comparison with ethanol, as associating effect is less important for 1-Octanol, it seems that there is no association between the two molecules. 
Table 5: Densities $(\rho)$ and excess molar volumes $\left(v^{E}\right)$ for furan $(1)+1$-Octanol(2) binary system as a function of furan mole fraction at atmospheric pressure $\left(U_{p}= \pm 0.03\right.$ $\left.\mathrm{kPa}, \mathrm{U}_{\mathrm{x} 1}= \pm 2 \times 10^{-5}, \mathrm{U}_{\mathrm{T}}= \pm 0.01 \mathrm{~K}, \mathrm{U}_{\mathrm{\rho}}= \pm 10^{-5} \mathrm{~g} \cdot \mathrm{cm}^{-3}, \mathrm{U}_{\mathrm{VE}}= \pm 0.003 \mathrm{~cm}^{3} \cdot \mathrm{mol}^{-1}(\mathrm{k}=2)\right)$.

\begin{tabular}{|c|c|c|c|c|c|c|c|c|c|c|c|c|}
\hline$x_{1}$ & $d / \mathrm{g} \cdot \mathrm{cm}^{-3}$ & $\begin{array}{c}V^{E} \\
/ \mathrm{cm}^{3} \cdot \mathrm{mol}^{-1} \\
\end{array}$ & $d /$ g.cm $\mathrm{cm}^{-3}$ & $\begin{array}{c}V^{E} \\
/ \mathrm{cm}^{3} \cdot \mathrm{mol}^{-1} \\
\end{array}$ & $d / \mathrm{g} \cdot \mathrm{cm}^{-3}$ & $\begin{array}{c}V^{E} \\
/ \mathrm{cm}^{3} \cdot \mathrm{mol}^{-1} \\
\end{array}$ & $d / \mathrm{g} \cdot \mathrm{cm}^{-3}$ & $\begin{array}{c}V^{E} \\
/ \mathrm{cm}^{3} \cdot \mathrm{mol}^{-1} \\
\end{array}$ & $d / \mathrm{g} \cdot \mathrm{cm}^{-1}$ & $\begin{array}{c}V^{E} \\
/ \mathrm{cm}^{3} \cdot \mathrm{mol}^{-}\end{array}$ & $\begin{array}{c}d \\
/ \mathrm{g} \cdot \mathrm{cm}^{-3} \\
\end{array}$ & $\begin{array}{c}V^{E} \\
/ \mathrm{cm}^{3} \cdot \mathrm{mol}^{-}\end{array}$ \\
\hline & \multicolumn{2}{|c|}{$\mathrm{T}=278.15 \mathrm{~K}$} & \multicolumn{2}{|c|}{$\mathrm{T}=283.15 \mathrm{~K}$} & \multicolumn{2}{|c|}{$\mathrm{T}=288.15 \mathrm{~K}$} & \multicolumn{2}{|c|}{$\mathrm{T}=293.15 \mathrm{~K}$} & \multicolumn{2}{|c|}{$\mathrm{T}=298.15 \mathrm{~K}$} & \multicolumn{2}{|c|}{$\mathrm{T}=303.15 \mathrm{~K}$} \\
\hline 0.0134 & 0.8361 & 0.0123 & 0.83262 & 0.013 & 0.82918 & 0.013 & 0.82574 & 0.010 & 0.82227 & 0.010 & 0.8188 & 0.010 \\
\hline 0.0256 & 0.8367 & 0.0234 & 0.83325 & 0.023 & 0.82979 & 0.024 & 0.82632 & 0.023 & 0.82284 & 0.022 & 0.8193 & 0.022 \\
\hline 0.0506 & 0.8380 & 0.0449 & 0.83456 & 0.043 & 0.83106 & 0.045 & 0.82755 & 0.045 & 0.82403 & 0.045 & 0.8205 & 0.044 \\
\hline 0.0739 & 0.8393 & 0.0702 & 0.83578 & 0.070 & 0.83225 & 0.071 & 0.82871 & 0.071 & 0.82517 & 0.067 & 0.8216 & 0.070 \\
\hline 0.0995 & 0.8408 & 0.0888 & 0.83721 & 0.089 & 0.83365 & 0.089 & 0.83007 & 0.088 & 0.82647 & 0.089 & 0.8229 & 0.089 \\
\hline 0.1495 & 0.8437 & 0.1310 & 0.8401 & 0.130 & 0.83645 & 0.132 & 0.83279 & 0.132 & 0.82911 & 0.132 & 0.8254 & 0.132 \\
\hline 0.1999 & 0.8469 & 0.1667 & 0.84322 & 0.167 & 0.83948 & 0.170 & 0.83574 & 0.169 & 0.83200 & 0.165 & 0.8282 & 0.170 \\
\hline 0.2512 & 0.8504 & 0.2043 & 0.8466 & 0.205 & 0.84278 & 0.206 & 0.83894 & 0.206 & 0.83506 & 0.209 & 0.8312 & 0.208 \\
\hline 0.3042 & 0.8543 & 0.2351 & 0.85038 & 0.236 & 0.84644 & 0.240 & 0.84250 & 0.239 & 0.83852 & 0.241 & 0.8345 & 0.241 \\
\hline 0.3501 & 0.8578 & 0.2661 & 0.85382 & 0.269 & 0.84980 & 0.271 & 0.84575 & 0.273 & 0.84170 & 0.270 & 0.8376 & 0.273 \\
\hline 0.4043 & 0.8624 & 0.2942 & 0.85824 & 0.298 & 0.85409 & 0.301 & 0.84992 & 0.302 & 0.84573 & 0.302 & 0.8415 & 0.302 \\
\hline 0.4511 & 0.8666 & 0.3143 & 0.86237 & 0.316 & 0.85810 & 0.320 & 0.85382 & 0.320 & 0.84951 & 0.321 & 0.8452 & 0.320 \\
\hline 0.5000 & 0.8714 & 0.3297 & 0.86702 & 0.333 & 0.86263 & 0.336 & 0.85822 & 0.336 & 0.85380 & 0.334 & 0.8493 & 0.337 \\
\hline 0.5529 & 0.8770 & 0.3396 & 0.87251 & 0.342 & 0.86797 & 0.345 & 0.86340 & 0.346 & 0.85881 & 0.345 & 0.8542 & 0.346 \\
\hline 0.6016 & 0.8827 & 0.3437 & 0.87802 & 0.346 & 0.87332 & 0.350 & 0.86860 & 0.350 & 0.86386 & 0.349 & 0.8591 & 0.349 \\
\hline 0.6500 & 0.8888 & 0.3384 & 0.88401 & 0.340 & 0.87915 & 0.343 & 0.87427 & 0.343 & 0.86934 & 0.343 & 0.8644 & 0.342 \\
\hline 0.7000 & 0.8959 & 0.3205 & 0.89086 & 0.323 & 0.88581 & 0.325 & 0.88073 & 0.326 & 0.87564 & 0.322 & 0.8705 & 0.325 \\
\hline 0.7499 & 0.9035 & 0.3004 & 0.89833 & 0.302 & 0.89307 & 0.304 & 0.88778 & 0.305 & 0.88246 & 0.303 & 0.8771 & 0.303 \\
\hline 0.8010 & 0.9123 & 0.2674 & 0.90685 & 0.269 & 0.90135 & 0.272 & 0.89582 & 0.271 & 0.89024 & 0.271 & 0.8846 & 0.271 \\
\hline 0.8500 & 0.9218 & 0.2186 & 0.91605 & 0.220 & 0.91029 & 0.222 & 0.90449 & 0.222 & 0.89867 & 0.220 & 0.8927 & 0.223 \\
\hline 0.9010 & 0.9327 & 0.1604 & 0.92673 & 0.161 & 0.92067 & 0.163 & 0.91456 & 0.164 & 0.90840 & 0.163 & 0.9022 & 0.165 \\
\hline 0.9250 & 0.9385 & 0.1223 & 0.9323 & 0.123 & 0.92608 & 0.125 & 0.91981 & 0.126 & 0.91349 & 0.125 & 0.9071 & 0.127 \\
\hline 0.9500 & 0.9447 & 0.0856 & 0.93839 & 0.086 & 0.93199 & 0.088 & 0.92554 & 0.089 & 0.91903 & 0.089 & 0.9125 & 0.089 \\
\hline 0.9750 & 0.9514 & 0.0423 & 0.94493 & 0.042 & 0.93835 & 0.043 & 0.93171 & 0.044 & 0.92501 & 0.044 & 0.9183 & 0.044 \\
\hline
\end{tabular}


Table 6: Redlich-Kister parameters and deviation for furan + 1-Octanol binary system.

\begin{tabular}{ccccc}
\hline \multirow{2}{*}{$\mathbf{T} / \mathbf{K}$} & \multicolumn{3}{c}{ Redlich Kister parameters } & $\begin{array}{c}\text { Variance } \\
\text { \%, equation }\end{array}$ \\
\cline { 2 - 4 } & $\mathbf{A}_{\mathbf{0}}$ & $\mathbf{A}_{\mathbf{1}}$ & $\mathbf{A}_{\mathbf{2}}$ & $\mathbf{7}$ \\
\hline 278.15 & 1.318 & 0.512 & 0.095 & 0.002 \\
283.15 & 1.329 & 0.517 & 0.080 & 0.002 \\
288.15 & 1.341 & 0.518 & 0.087 & 0.002 \\
293.15 & 1.344 & 0.523 & 0.077 & 0.003 \\
298.15 & 1.341 & 0.515 & 0.072 & 0.002 \\
303.15 & 1.343 & 0.516 & 0.090 & 0.002 \\
\hline
\end{tabular}

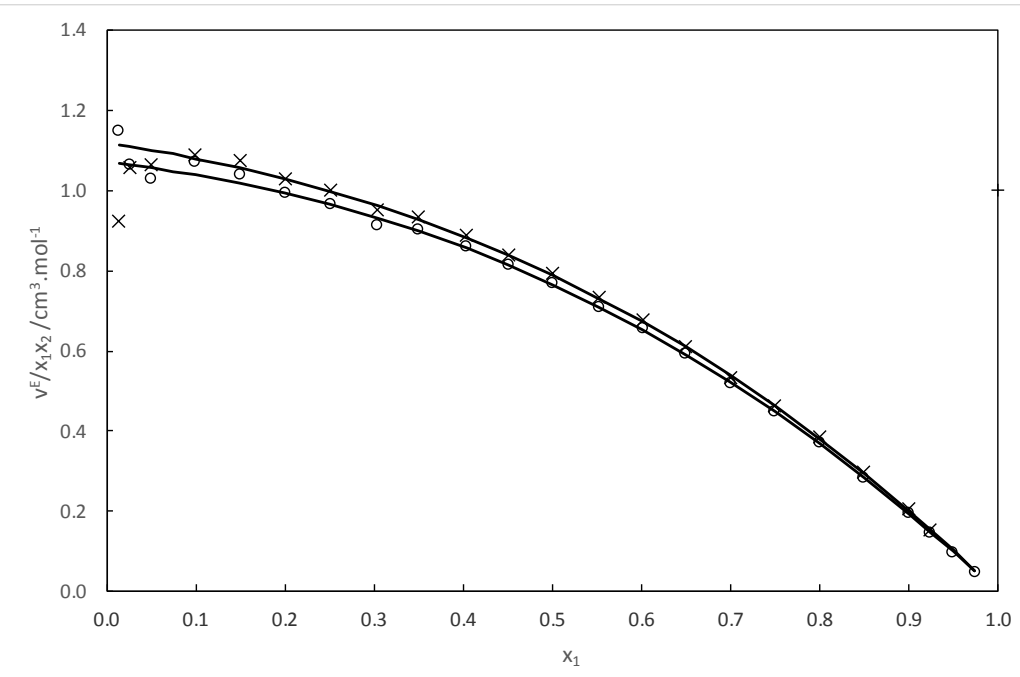

Figure 3: $\frac{v^{E}}{x_{1} x_{2}}$ for furan(1) + 1-Octanol(2) system as a function of furan mole fraction at atmospheric pressure and 2 different temperatures: (o) $283.15 \mathrm{~K},(x) 303.15 \mathrm{~K}$, solid line: RedlichKister correlation. 


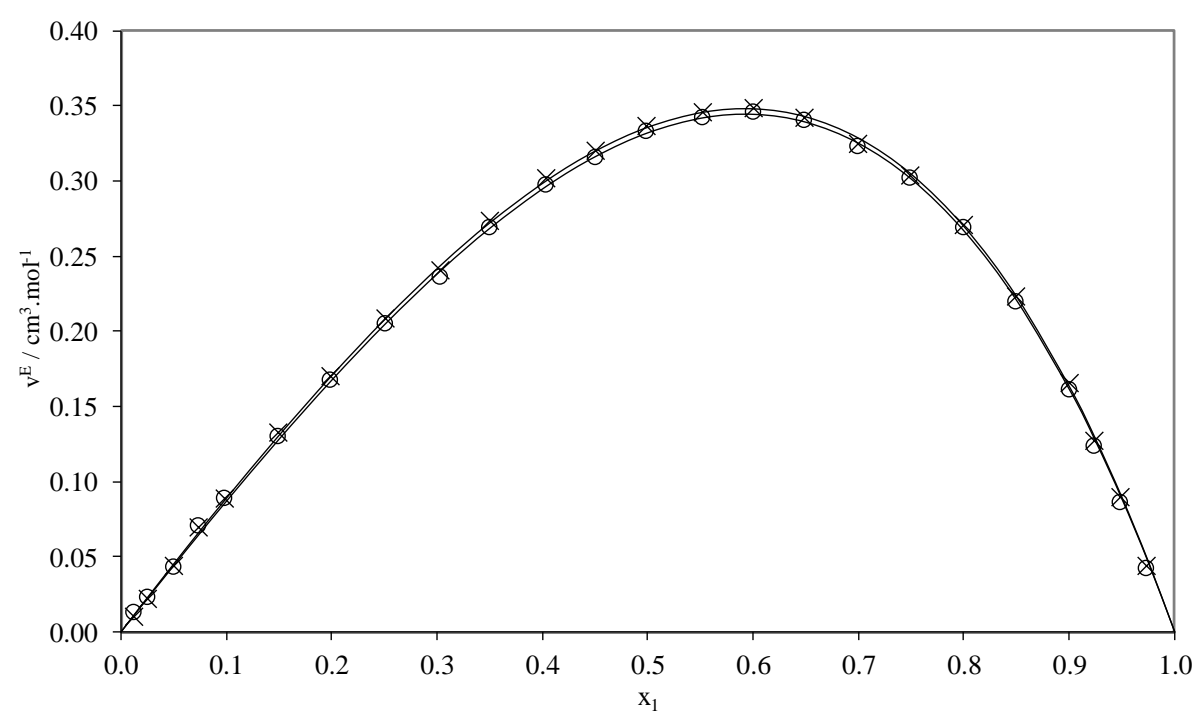

Figure 4: Excess molar volume $\left(v^{E}\right)$ for furan $(1)+1-O c t a n o l(2)$ system as a function of furan mole fraction at atmospheric pressure and 2 different temperatures: $(0) 283.15 \mathrm{~K},(x) 303.15 \mathrm{~K}$, solid line: Redlich Kister correlation.

In order to understand the difference between excess volumes of Furan-Ethanol and Furan - 1Octanol, we draw a parallel with the mixtures Benzene - Alcohols. First, we can consider that the presence of the oxygen atom in the aromatic ring is the major difference between Furan and Benzene. Excess Volume with Benzene is essentially positive and is increasing with the length of the 1-Octanol, probably because of steric considerations. Thus, we can conclude that negative excess volume of Furan-Ethanol is due to H-Bond between Ethanol and the oxygen atom of Furan. Indeed, the alkyl chain of 1-Octanol may hide the potential H-Bond, leading to positive excess volume of Furan-1-Octanol. This assumption is comforted by the increase of excess volume with temperature, traducing the $\mathrm{H}$-bonding breaking when the temperature increases.

\section{c. Eugenol 1-Octanol binary system}

Table 7 presents the values of mixtures densities and excess volume. Table 8 presents our results. Following Desnoyers and Perron's method we have considered 3 RK parameters. Figs 5-6 presents respectively, $\frac{v^{E}}{x_{1} x_{2}}$ as a function of $\mathrm{x}_{1}$ and $\mathrm{v}^{\mathrm{E}}$ as a function of $\mathrm{x}_{1}$, at 2 different temperatures. There is no particular behavior. We can consider that it is a mixture of two liquids with similar size. As we can see, excess molar volume is negative, it is certainly due to dipole-dipole interaction as the dipole moment of the two chemicals are quite similar (eugenol: 1.129 D [7] and 1-Octanol: 1.66 D [6]). 
Table 7: Densities $(\rho)$ and excess molar volumes $\left(v^{E}\right)$ for Eugenol(1) + 1-Octanol(2) binary system as a function of furan mole fraction at atmospheric pressure $\left(U_{\mathrm{p}}= \pm 0.03 \mathrm{kPa}, \mathrm{U}_{\mathrm{x} 1}= \pm 2 \times 10^{-5}, \mathrm{U}_{\mathrm{T}}= \pm 0.01 \mathrm{~K}, \mathrm{U}_{\rho}= \pm 10^{-5} \mathrm{~g} \cdot \mathrm{cm}^{-3}, \mathrm{U}_{\mathrm{VE}}= \pm 0.003 \mathrm{~cm}^{3} \cdot \mathrm{mol}^{-1}(\mathrm{k}=2)\right)$.

\begin{tabular}{|c|c|c|c|c|c|c|c|c|c|c|}
\hline$x_{1}$ & $\rho / \mathrm{g} \cdot \mathrm{cm}^{-3}$ & $\begin{array}{c}v^{E} \\
/ \mathrm{cm}^{3} \cdot \mathrm{mol}^{-1} \\
\end{array}$ & $\rho / \mathrm{g} \cdot \mathrm{cm}^{-3}$ & $\begin{array}{c}v^{E} \\
/ \mathrm{cm}^{3} \cdot \mathrm{mol}^{-1} \\
\end{array}$ & $\rho / \mathrm{g} \cdot \mathrm{cm}^{-3}$ & $\begin{array}{c}v^{E} \\
/ \mathrm{cm}^{3} \cdot \mathrm{mol}^{-1} \\
\end{array}$ & $\rho / \mathrm{g} \cdot \mathrm{cm}^{-3}$ & $\begin{array}{c}v^{E} \\
/ \mathrm{cm}^{3} \cdot \mathrm{mol}^{-1} \\
\end{array}$ & $\rho /$ g.cm ${ }^{-3}$ & $\begin{array}{c}v^{E} \\
/ \mathrm{cm}^{3} \cdot \mathrm{mol}^{-1} \\
\end{array}$ \\
\hline & \multicolumn{2}{|c|}{$\mathrm{T}=283.15 \mathrm{~K}$} & \multicolumn{2}{|c|}{$\mathrm{T}=288.15 \mathrm{~K}$} & \multicolumn{2}{|c|}{$\mathrm{T}=293.15 \mathrm{~K}$} & \multicolumn{2}{|c|}{$\mathrm{T}=298.15 \mathrm{~K}$} & \multicolumn{2}{|c|}{$\mathrm{T}=303.15 \mathrm{~K}$} \\
\hline 0.0500 & 0.84454 & -0.149 & 0.84104 & -0.142 & 0.83754 & -0.137 & 0.83403 & -0.138 & 0.8305 & -0.127 \\
\hline 0.1001 & 0.85699 & -0.242 & 0.85342 & -0.233 & 0.84984 & -0.223 & 0.84623 & -0.215 & 0.8426 & -0.206 \\
\hline 0.1500 & 0.86955 & -0.356 & 0.86591 & -0.344 & 0.86224 & -0.328 & 0.85857 & -0.318 & 0.8549 & -0.304 \\
\hline 0.1502 & 0.86934 & -0.310 & 0.86570 & -0.297 & 0.86205 & -0.285 & 0.85837 & -0.273 & 0.8547 & -0.259 \\
\hline 0.2002 & 0.88188 & -0.406 & 0.87817 & -0.391 & 0.87444 & -0.374 & 0.87069 & -0.359 & 0.8669 & -0.342 \\
\hline 0.2502 & 0.89431 & -0.479 & 0.89053 & -0.461 & 0.88674 & -0.443 & 0.88292 & -0.425 & 0.8791 & -0.404 \\
\hline 0.3004 & 0.90676 & -0.534 & 0.90291 & -0.513 & 0.89905 & -0.492 & 0.89516 & -0.471 & 0.8913 & -0.451 \\
\hline 0.3502 & 0.91897 & -0.562 & 0.91506 & -0.540 & 0.91114 & -0.518 & 0.90719 & -0.495 & 0.9032 & -0.473 \\
\hline 0.4001 & 0.9312 & -0.585 & 0.92723 & -0.563 & 0.92325 & -0.539 & 0.91925 & -0.516 & 0.9152 & -0.493 \\
\hline 0.4003 & 0.93125 & -0.583 & 0.92728 & -0.561 & 0.92329 & -0.536 & 0.91929 & -0.513 & 0.9153 & -0.490 \\
\hline 0.4501 & 0.94351 & -0.610 & 0.93948 & -0.587 & 0.93545 & -0.563 & 0.93139 & -0.539 & 0.9273 & -0.514 \\
\hline 0.5002 & 0.95579 & -0.619 & 0.95171 & -0.597 & 0.94762 & -0.572 & 0.94353 & -0.552 & 0.9394 & -0.525 \\
\hline 0.5502 & 0.96788 & -0.598 & 0.96379 & -0.582 & 0.95966 & -0.559 & 0.95550 & -0.535 & 0.9513 & -0.512 \\
\hline 0.6000 & 0.97992 & -0.571 & 0.97576 & -0.551 & 0.97158 & -0.529 & 0.96738 & -0.506 & 0.9632 & -0.483 \\
\hline 0.6502 & 0.99207 & -0.543 & 0.98786 & -0.524 & 0.98364 & -0.503 & 0.97940 & -0.482 & 0.9752 & -0.461 \\
\hline 0.6992 & 1.0039 & -0.509 & 0.99965 & -0.491 & 0.99539 & -0.472 & 0.99113 & -0.455 & 0.9868 & -0.434 \\
\hline 0.7482 & 1.01573 & -0.470 & 1.01144 & -0.454 & 1.00715 & -0.438 & 1.00284 & -0.420 & 0.9985 & -0.404 \\
\hline 0.7984 & 1.02767 & -0.401 & 1.02335 & -0.388 & 1.01902 & -0.373 & 1.01468 & -0.358 & 1.0103 & -0.344 \\
\hline 0.8457 & 1.03902 & -0.347 & 1.03468 & -0.338 & 1.03032 & -0.325 & 1.02597 & -0.316 & 1.0216 & -0.302 \\
\hline 0.8997 & 1.05185 & -0.265 & 1.04747 & -0.257 & 1.04309 & -0.249 & 1.03870 & -0.241 & 1.0343 & -0.233 \\
\hline 0.9499 & 1.06377 & -0.182 & 1.05938 & -0.180 & 1.05497 & -0.174 & 1.05057 & -0.171 & 1.0462 & -0.167 \\
\hline
\end{tabular}




\begin{tabular}{|c|c|c|c|c|c|c|c|c|}
\hline$x_{1}$ & $\rho / \mathrm{g} \cdot \mathrm{cm}^{-3}$ & $\begin{array}{c}v^{E} \\
/ \mathrm{cm}^{3} \cdot \mathrm{mol}^{-1} \\
\end{array}$ & $\rho / \mathrm{g} \cdot \mathrm{cm}^{-3}$ & $\begin{array}{c}v^{E} \\
/ \mathrm{cm}^{3} \cdot \mathrm{mol}^{-1} \\
\end{array}$ & $\rho /$ g. $\mathrm{cm}^{-3}$ & $\begin{array}{c}v^{E} \\
/ \mathrm{cm}^{3} \cdot \mathrm{mol}^{-1} \\
\end{array}$ & $\rho /$ g.cm ${ }^{-3}$ & $\begin{array}{c}v^{E} \\
/ \mathrm{cm}^{3} \cdot \mathrm{mol}^{-1} \\
\end{array}$ \\
\hline & \multicolumn{2}{|c|}{$\mathrm{T}=308.15 \mathrm{~K}$} & \multicolumn{2}{|c|}{$\mathrm{T}=313.15 \mathrm{~K}$} & \multicolumn{2}{|c|}{$\mathrm{T}=318.15 \mathrm{~K}$} & \multicolumn{2}{|c|}{$\mathrm{T}=323.15 \mathrm{~K}$} \\
\hline 0.0500 & 0.82688 & -0.120 & 0.82329 & -0.114 & 0.81967 & -0.108 & 0.81603 & -0.102 \\
\hline 0.1001 & 0.83897 & -0.195 & 0.83530 & -0.184 & 0.83161 & -0.174 & 0.82789 & -0.161 \\
\hline 0.1500 & 0.85115 & -0.288 & 0.84741 & -0.274 & 0.84365 & -0.259 & 0.83986 & -0.243 \\
\hline 0.1502 & 0.85095 & -0.242 & 0.84721 & -0.227 & 0.84345 & -0.213 & 0.83966 & -0.196 \\
\hline 0.2002 & 0.86314 & -0.324 & 0.85933 & -0.305 & 0.85550 & -0.287 & 0.85165 & -0.268 \\
\hline 0.2502 & 0.87523 & -0.383 & 0.87137 & -0.364 & 0.86747 & -0.342 & 0.86355 & -0.319 \\
\hline 0.3004 & 0.88735 & -0.426 & 0.88342 & -0.404 & 0.87947 & -0.382 & 0.87549 & -0.356 \\
\hline 0.3502 & 0.89927 & -0.449 & 0.89528 & -0.425 & 0.89127 & -0.400 & 0.88724 & -0.374 \\
\hline 0.4001 & 0.91121 & -0.467 & 0.90717 & -0.443 & 0.90311 & -0.417 & 0.89904 & -0.392 \\
\hline 0.4003 & 0.91126 & -0.465 & 0.90722 & -0.441 & 0.90316 & -0.416 & 0.89908 & -0.389 \\
\hline 0.4501 & 0.92325 & -0.490 & 0.91915 & -0.464 & 0.91505 & -0.440 & 0.91092 & -0.412 \\
\hline 0.5002 & 0.93527 & -0.499 & 0.93113 & -0.474 & 0.92698 & -0.450 & 0.92281 & -0.424 \\
\hline 0.5502 & 0.94717 & -0.487 & 0.94298 & -0.462 & 0.93879 & -0.439 & 0.93457 & -0.412 \\
\hline 0.6000 & 0.95896 & -0.459 & 0.95474 & -0.437 & 0.95050 & -0.413 & 0.94625 & -0.388 \\
\hline 0.6502 & 0.97090 & -0.438 & 0.96664 & -0.417 & 0.96237 & -0.395 & 0.95808 & -0.371 \\
\hline 0.6992 & 0.98256 & -0.415 & 0.97826 & -0.395 & 0.97395 & -0.373 & 0.96964 & -0.353 \\
\hline 0.7482 & 0.99421 & -0.385 & 0.98988 & -0.368 & 0.98554 & -0.349 & 0.98120 & -0.330 \\
\hline 0.7984 & 1.00599 & -0.328 & 1.00163 & -0.313 & 0.99727 & -0.297 & 0.99290 & -0.280 \\
\hline 0.8457 & 1.01722 & -0.290 & 1.01284 & -0.278 & 1.00845 & -0.264 & 1.00406 & -0.251 \\
\hline 0.8997 & 1.02991 & -0.224 & 1.02552 & -0.218 & 1.02111 & -0.208 & 1.01670 & -0.198 \\
\hline 0.9499 & 1.04174 & -0.162 & 1.03733 & -0.159 & 1.03291 & -0.154 & 1.02849 & -0.149 \\
\hline
\end{tabular}


Table 8: Redlich-Kister parameters and deviation for eugenol + 1-Octanol binary system.

\begin{tabular}{|c|c|c|c|c|}
\hline \multirow{2}{*}{$\mathbf{T} / \mathbf{K}$} & \multicolumn{3}{|c|}{ Redlich Kister parameters } & \multirow{2}{*}{$\begin{array}{c}\text { Variance } \\
\sigma \text {, equation } \\
7\end{array}$} \\
\hline & $\mathbf{A}_{0}$ & $\mathbf{A}_{1}$ & $\mathbf{A}_{2}$ & \\
\hline 283.15 & -2.408 & 0.034 & -0.479 & 0.017 \\
\hline 288.15 & -2.318 & -0.001 & -0.529 & 0.018 \\
\hline 293.15 & -2.222 & -0.012 & -0.515 & 0.017 \\
\hline 298.15 & -2.127 & -0.019 & -0.539 & 0.018 \\
\hline 303.15 & -2.030 & -0.029 & -0.527 & 0.017 \\
\hline 308.15 & -1.929 & -0.047 & -0.506 & 0.017 \\
\hline 313.15 & -1.830 & -0.065 & -0.503 & 0.018 \\
\hline 318.15 & -1.730 & -0.075 & -0.482 & 0.018 \\
\hline 323.15 & -1.624 & -0.095 & -0.462 & 0.018 \\
\hline
\end{tabular}

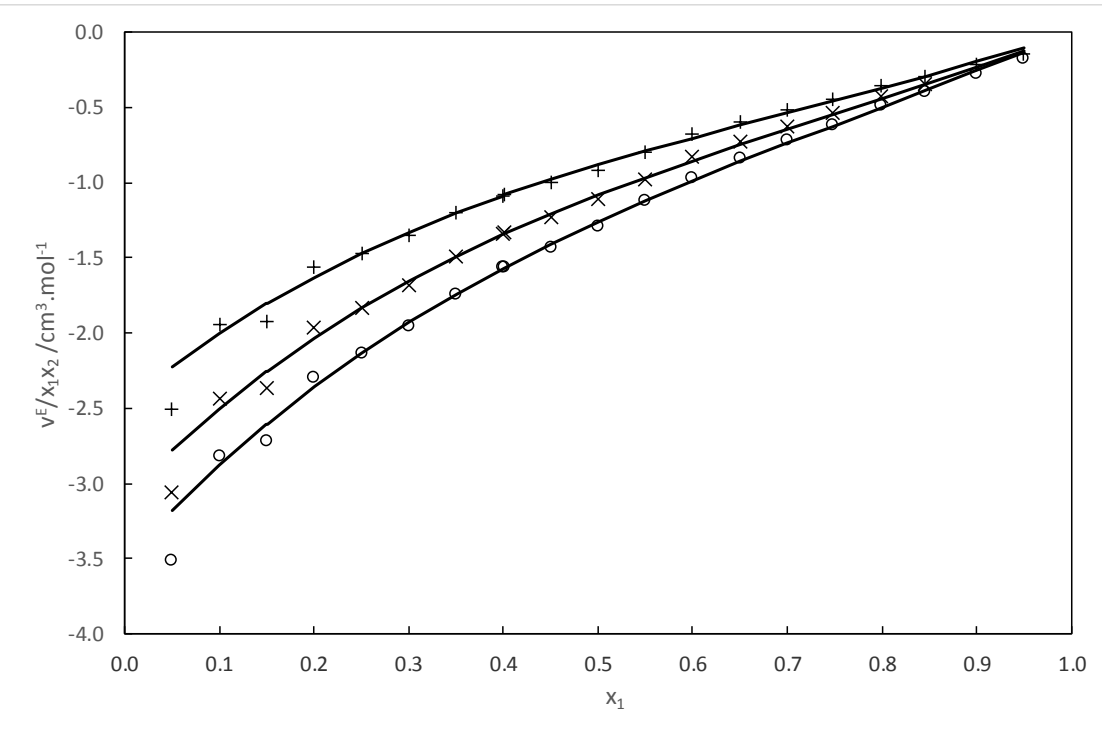

Figure 5: $\frac{v^{E}}{x_{1} x_{2}}$ for eugenol(1) + 1-Octanol(2) system as a function of eugenol mole fraction at atmospheric pressure and 2 different temperatures: (o) $283.15 \mathrm{~K},(x) 303.15 \mathrm{~K},(+) 323.15 \mathrm{~K}$, solid line: Redlich-Kister correlation. 


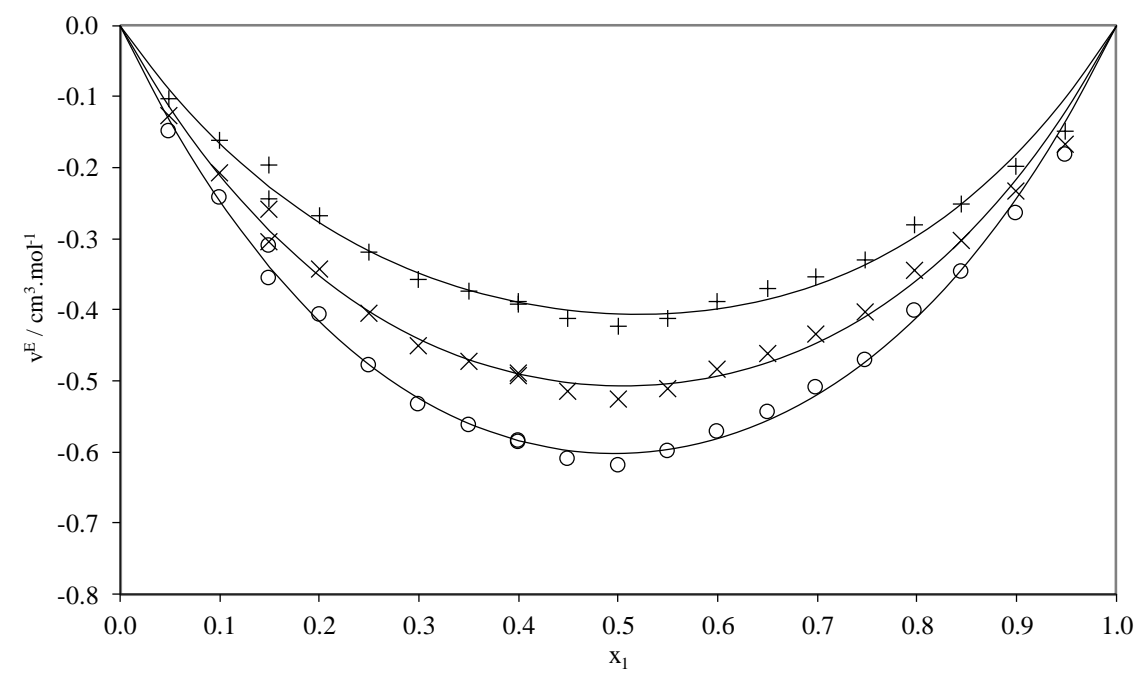

Figure 6: Excess molar volume $\left(v^{E}\right)$ for eugenol(1) + 1-Octanol(2) system as a function of eugenol mole fraction at atmospheric pressure and 3 different temperatures: $(0) 283.15 K,(x) 303.15 K,(+)$ 323.15K, solid line: Redlich-Kister correlation.

\section{d. Eugenol n-hexane binary system}

Table 9 presents the values of mixtures densities and excess volume. Table 10 presents our results. Following Desnoyers and Perron's method we have considered 5 RK parameters. Figs 7-8 presents respectively, $\frac{v^{E}}{x_{1} x_{2}}$ as a function of $x_{1}$ and $v^{E}$ as a function of $x_{1}$, at 2 different temperatures. There is no particular behavior. As we can see, excess molar volume is negative, it is a surprising result as the two dipole moments of the two chemicals are very different (eugenol: 1.129 D and no dipole moment for $n$-hexane [6]). Another type of molecular interaction must be predominant. 
Table 9: Densities $(\rho)$ and excess molar volumes $\left(v^{E}\right)$ for Eugenol(1) $+\mathrm{n}$-hexane (2) binary system as a function of furan mole fraction at atmospheric pressure $\left(U_{\mathrm{p}}= \pm 0.03 \mathrm{kPa}, \mathrm{U}_{\mathrm{x} 1}= \pm 2 \times 10^{-5}, \mathrm{U}_{\mathrm{T}}= \pm 0.01 \mathrm{~K}, \mathrm{U}_{\rho}= \pm 10^{-5} \mathrm{~g} \cdot \mathrm{cm}^{-3}, \mathrm{U}_{\mathrm{VE}}= \pm 0.003 \mathrm{~cm}^{3} \cdot \mathrm{mol}^{-1}(\mathrm{k}=2)\right)$.

\begin{tabular}{|c|c|c|c|c|c|c|c|c|c|c|}
\hline \multirow[t]{2}{*}{$x_{1}$} & $\rho /$ g.cm ${ }^{-3}$ & $\begin{array}{c}v^{E} \\
/ \mathrm{cm}^{3} \cdot \mathrm{mol}^{-1} \\
\end{array}$ & $\rho /$ g.cm ${ }^{-3}$ & $\begin{array}{c}v^{E} \\
/ \mathrm{cm}^{3} \cdot \mathrm{mol}^{-1} \\
\end{array}$ & $\rho / \mathrm{g} \cdot \mathrm{cm}^{-3}$ & $\begin{array}{c}v^{E} \\
/ \mathrm{cm}^{3} \cdot \mathrm{mol}^{-1} \\
\end{array}$ & $\rho /$ g.cm ${ }^{-3}$ & $\begin{array}{c}v^{E} \\
/ \mathrm{cm}^{3} \cdot \mathrm{mol}^{-1} \\
\end{array}$ & $\rho / \mathrm{g} \cdot \mathrm{cm}^{-3}$ & $\begin{array}{c}v^{E} \\
/ \mathrm{cm}^{3} \cdot \mathrm{mol}^{-1} \\
\end{array}$ \\
\hline & \multicolumn{2}{|c|}{$\mathrm{T}=283.15 \mathrm{~K}$} & \multicolumn{2}{|c|}{$\mathrm{T}=288.15 \mathrm{~K}$} & \multicolumn{2}{|c|}{$\mathbf{T}=293.15 \mathrm{~K}$} & \multicolumn{2}{|c|}{$\mathrm{T}=298.15 \mathrm{~K}$} & \multicolumn{2}{|c|}{$T=303.15 \mathrm{~K}$} \\
\hline 0.0500 & 0.69309 & -0.068 & 0.68862 & -0.076 & 0.68413 & -0.089 & 0.67961 & -0.105 & 0.67506 & -0.119 \\
\hline 0.1000 & 0.71694 & -0.147 & 0.71248 & -0.166 & 0.70797 & -0.185 & 0.70343 & -0.207 & 0.69887 & -0.229 \\
\hline 0.1500 & 0.74038 & -0.222 & 0.73590 & -0.245 & 0.73138 & -0.270 & 0.72685 & -0.301 & 0.72229 & -0.331 \\
\hline 0.2000 & 0.76344 & -0.299 & 0.75894 & -0.325 & 0.75444 & -0.360 & 0.74991 & -0.397 & 0.74535 & -0.433 \\
\hline 0.2500 & 0.78602 & -0.362 & 0.78153 & -0.394 & 0.77703 & -0.433 & 0.77250 & -0.474 & 0.76794 & -0.515 \\
\hline 0.3000 & 0.80828 & -0.433 & 0.80379 & -0.469 & 0.79930 & -0.512 & 0.79477 & -0.556 & 0.79022 & -0.601 \\
\hline 0.3500 & 0.82996 & -0.474 & 0.82548 & -0.513 & 0.82098 & -0.557 & 0.81649 & -0.609 & 0.81193 & -0.654 \\
\hline 0.4000 & 0.85138 & -0.523 & 0.84691 & -0.565 & 0.84241 & -0.609 & 0.83790 & -0.659 & 0.83337 & -0.708 \\
\hline 0.4491 & 0.87192 & -0.555 & 0.86745 & -0.597 & 0.86298 & -0.646 & 0.85846 & -0.692 & 0.85394 & -0.743 \\
\hline 0.5000 & 0.89289 & -0.585 & 0.88842 & -0.627 & 0.88394 & -0.672 & 0.87945 & -0.722 & 0.87495 & -0.773 \\
\hline 0.5489 & 0.91254 & -0.594 & 0.90807 & -0.633 & 0.90360 & -0.678 & 0.89910 & -0.723 & 0.89460 & -0.772 \\
\hline 0.6000 & 0.9326 & -0.581 & 0.92814 & -0.619 & 0.92367 & -0.660 & 0.91918 & -0.703 & 0.91469 & -0.750 \\
\hline 0.6473 & 0.95086 & -0.571 & 0.94641 & -0.607 & 0.94195 & -0.646 & 0.93747 & -0.687 & 0.93299 & -0.730 \\
\hline 0.6994 & 0.97047 & -0.533 & 0.96602 & -0.565 & 0.96157 & -0.600 & 0.95709 & -0.635 & 0.95261 & -0.673 \\
\hline 0.7464 & 0.98787 & -0.496 & 0.98343 & -0.525 & 0.97898 & -0.556 & 0.97451 & -0.587 & 0.97004 & -0.620 \\
\hline 0.8000 & 1.00726 & -0.443 & 1.00283 & -0.469 & 0.99839 & -0.494 & 0.99393 & -0.520 & 0.98947 & -0.548 \\
\hline 0.8499 & 1.02501 & -0.389 & 1.02057 & -0.407 & 1.01613 & -0.427 & 1.01169 & -0.449 & 1.00723 & -0.469 \\
\hline 0.8991 & 1.04192 & -0.298 & 1.03750 & -0.313 & 1.03307 & -0.327 & 1.02863 & -0.341 & 1.02419 & -0.357 \\
\hline 0.9498 & 1.05904 & -0.201 & 1.05463 & -0.211 & 1.05021 & -0.218 & 1.04578 & -0.226 & 1.04134 & -0.233 \\
\hline 0.9751 & 1.06733 & -0.134 & 1.06291 & -0.138 & 1.05849 & -0.141 & 1.05407 & -0.146 & 1.04965 & -0.151 \\
\hline
\end{tabular}




\begin{tabular}{|c|c|c|c|c|c|c|c|c|}
\hline$x_{1}$ & $\rho /$ g.cm ${ }^{-3}$ & $\begin{array}{c}v^{E} \\
/ \mathrm{cm}^{3} \cdot \mathrm{mol}^{-1}\end{array}$ & $\rho / \mathrm{g} \cdot \mathrm{cm}^{-3}$ & $\begin{array}{c}v^{E} \\
/ \mathrm{cm}^{3} \cdot \mathrm{mol}^{-1} \\
\end{array}$ & $\rho / \mathrm{g} \cdot \mathrm{cm}^{-3}$ & $\begin{array}{c}v^{E} \\
/ \mathrm{cm}^{3} \cdot \mathrm{mol}^{-1} \\
\end{array}$ & $\rho /$ g.cm ${ }^{-3}$ & $\begin{array}{c}v^{E} \\
/ \mathrm{cm}^{3} \cdot \mathrm{mol}^{-1}\end{array}$ \\
\hline & \multicolumn{2}{|c|}{$\mathrm{T}=308.15 \mathrm{~K}$} & \multicolumn{2}{|c|}{$\mathrm{T}=313.15 \mathrm{~K}$} & \multicolumn{2}{|c|}{$\mathrm{T}=318.15 \mathrm{~K}$} & \multicolumn{2}{|c|}{$\mathrm{T}=323.15 \mathrm{~K}$} \\
\hline 0.0500 & 0.67046 & -0.134 & 0.66583 & -0.149 & 0.66115 & -0.166 & 0.65644 & -0.188 \\
\hline 0.1000 & 0.69428 & -0.255 & 0.68966 & -0.282 & 0.68500 & -0.314 & 0.68029 & -0.346 \\
\hline 0.1500 & 0.71770 & -0.365 & 0.71308 & -0.400 & 0.70843 & -0.441 & 0.70374 & -0.485 \\
\hline 0.2000 & 0.74077 & -0.474 & 0.73616 & -0.517 & 0.73152 & -0.566 & 0.72685 & -0.619 \\
\hline 0.2500 & 0.76337 & -0.562 & 0.75877 & -0.611 & 0.75414 & -0.665 & 0.74949 & -0.725 \\
\hline 0.3000 & 0.78565 & -0.651 & 0.78107 & -0.706 & 0.77646 & -0.766 & 0.77182 & -0.830 \\
\hline 0.3500 & 0.80737 & -0.706 & 0.80280 & -0.764 & 0.79820 & -0.826 & 0.79358 & -0.893 \\
\hline 0.4000 & 0.82883 & -0.764 & 0.82427 & -0.823 & 0.81969 & -0.887 & 0.81508 & -0.955 \\
\hline 0.4491 & 0.84940 & -0.797 & 0.84485 & -0.856 & 0.84029 & -0.921 & 0.83570 & -0.990 \\
\hline 0.5000 & 0.87040 & -0.824 & 0.86586 & -0.881 & 0.86131 & -0.945 & 0.85675 & -1.014 \\
\hline 0.5489 & 0.89009 & -0.825 & 0.88557 & -0.882 & 0.88104 & -0.944 & 0.87649 & -1.010 \\
\hline 0.6000 & 0.91020 & -0.801 & 0.90570 & -0.857 & 0.90118 & -0.914 & 0.89664 & -0.975 \\
\hline 0.6473 & 0.92850 & -0.777 & 0.92401 & -0.828 & 0.91950 & -0.881 & 0.91498 & -0.939 \\
\hline 0.6994 & 0.94813 & -0.715 & 0.94365 & -0.761 & 0.93915 & -0.808 & 0.93465 & -0.860 \\
\hline 0.7464 & 0.96557 & -0.657 & 0.96109 & -0.697 & 0.95661 & -0.739 & 0.95212 & -0.784 \\
\hline 0.8000 & 0.98500 & -0.577 & 0.98054 & -0.611 & 0.97606 & -0.644 & 0.97159 & -0.682 \\
\hline 0.8499 & 1.00278 & -0.493 & 0.99832 & -0.518 & 0.99386 & -0.545 & 0.98939 & -0.573 \\
\hline 0.8991 & 1.01974 & -0.372 & 1.01529 & -0.390 & 1.01084 & -0.408 & 1.00639 & -0.428 \\
\hline 0.9498 & 1.03690 & -0.240 & 1.03247 & -0.251 & 1.02803 & -0.260 & 1.02359 & -0.270 \\
\hline 0.9751 & 1.04522 & -0.155 & 1.04079 & -0.161 & 1.03635 & -0.165 & 1.03192 & -0.170 \\
\hline
\end{tabular}


Table 10: Redlich-Kister parameters and deviation for eugenol $+\mathbf{n}$-hexane binary system.

\begin{tabular}{|c|c|c|c|c|c|c|}
\hline \multirow{2}{*}{$\mathbf{T} / \mathbf{K}$} & \multicolumn{5}{|c|}{ Redlich Kister parameters } & \multirow{2}{*}{$\begin{array}{c}\text { Variance } \\
\sigma, \text { equation } \\
7\end{array}$} \\
\hline & A0 & A1 & A2 & $\mathbf{A 3}$ & A4 & \\
\hline 283.15 & -2.287 & -0.454 & -0.229 & -1.065 & -0.169 & 0.016 \\
\hline 288.15 & -2.452 & -0.429 & -0.234 & -1.085 & -0.178 & 0.016 \\
\hline 293.15 & -2.634 & -0.386 & -0.238 & -1.081 & -0.180 & 0.016 \\
\hline 298.15 & -2.826 & -0.325 & -0.262 & -1.092 & -0.188 & 0.016 \\
\hline 303.15 & -3.024 & -0.298 & -0.264 & -1.046 & -0.200 & 0.017 \\
\hline 308.15 & -3.240 & -0.254 & -0.288 & -1.001 & -0.199 & 0.017 \\
\hline 313.15 & -3.474 & -0.208 & -0.306 & -0.986 & -0.203 & 0.017 \\
\hline 318.15 & -3.727 & -0.146 & -0.326 & -0.942 & -0.210 & 0.018 \\
\hline 323.15 & -3.997 & -0.079 & -0.363 & -0.893 & -0.214 & 0.018 \\
\hline
\end{tabular}

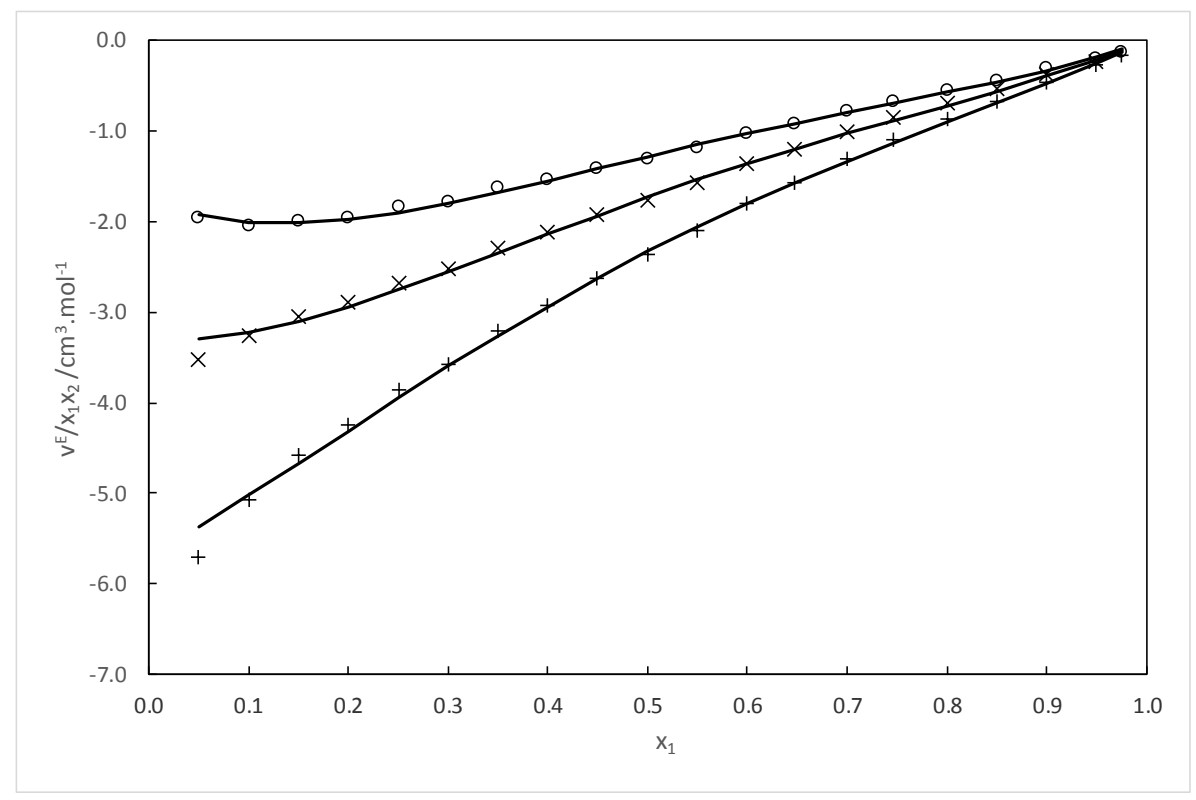

Figure 7: $\frac{v^{E}}{x_{1} x_{2}}$ for eugenol(1) $+\mathbf{n}$-hexane(2) system as a function of eugenol mole fraction at atmospheric pressure and 2 different temperatures: (0) $283.15 \mathrm{~K},(\times) 303.15 \mathrm{~K},(+) 323.15 \mathrm{~K}$, solid line: Redlich-Kister correlation. 


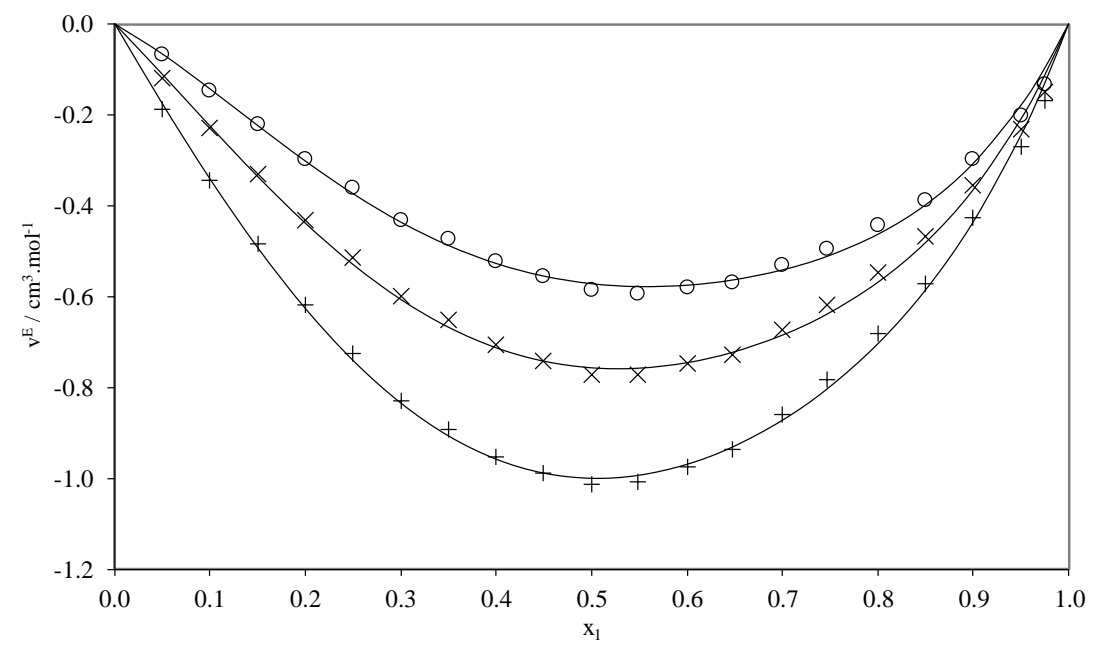

Figure 8: Excess molar volume $\left(v^{E}\right)$ for eugenol(1) $+n$-hexane (2) system as a function of eugenol mole fraction at atmospheric pressure and 3 different temperatures: $(0) 283.15 K,(x) 303.15 K,(+)$ $323.15 \mathrm{~K}$, solid line: Redlich-Kister correlation.

In order to have a better evaluation of the molecular interactions involved in such mixtures, we have decided to use the PFP model.

e. Partial molar volume

Using the parameters fitted for each binary system, we can plot the partial molar of furan or eugenol as a function of the molar composition: Figs. 9-12. We have also plotted on the same figure $v_{1, \varphi}$ the apparent molar volume for the furan and eugenol. Apparent molar volume is an important property as it is linked to the nature of the molecular interaction between the two molecules, herein the solute (furan or eugenol) and the solvent (n-Hexane, Ethanol and n-Octanol). The partial molar volume corresponds to the volume occupied by the solute in the solution and it depends to the nature of the surrounding molecules. Partial molar volume and apparent molar volume are linked by the equation 14.

$v_{i}=v_{\phi, i}+x_{i}\left(\frac{\partial v_{\phi, i}}{\partial n_{i}}\right)_{T P x_{j \neq i}}$ 
At infinite dilution, these two quantities are identical. The deviations seem to be more important for solute composition close to 0 . We can also mention that for the system Eugenol $+\mathrm{n}$-Hexane some important deviations exist in the region rich in n-hexane (Fig. 12). The deviations are more important, particularly at the highest temperature, in comparison with the others binary systems studied. There are two possibilities: it is necessary to increase the number of parameters of the Redlich Kister correlation (it is difficult to do that considering the experimental uncertainty and the fact that the curve $\frac{v^{E}}{x_{1} x_{2}}$ as a function of $\mathrm{x}_{1}$ are well correlated) or it may be the sign that molecular interactions are different for eugenol $+n$-hexane binary system in comparison with the others studied systems. Consequently, another model for the data treatment should be used in order to identify such kind of molecular interaction.

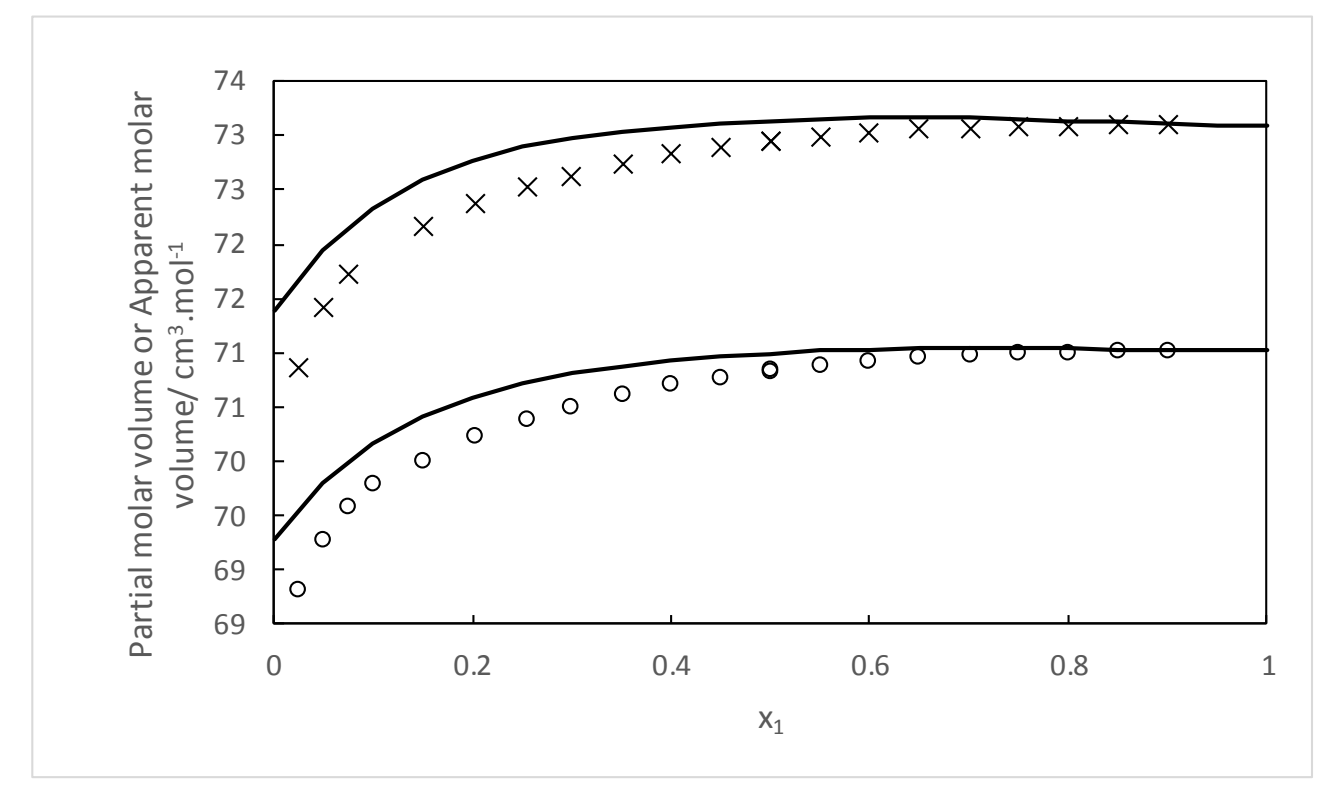

Figure 9: Calculated partial molar volume using Redlich-Kister correlation (solid line) and experimental apparent molar volume (symbols) of furan(1) + ethanol(2) system as a function of furan mole fraction at atmospheric pressure and 2 different temperatures: (0) $283.15 K,(x) 303.15$ K. 


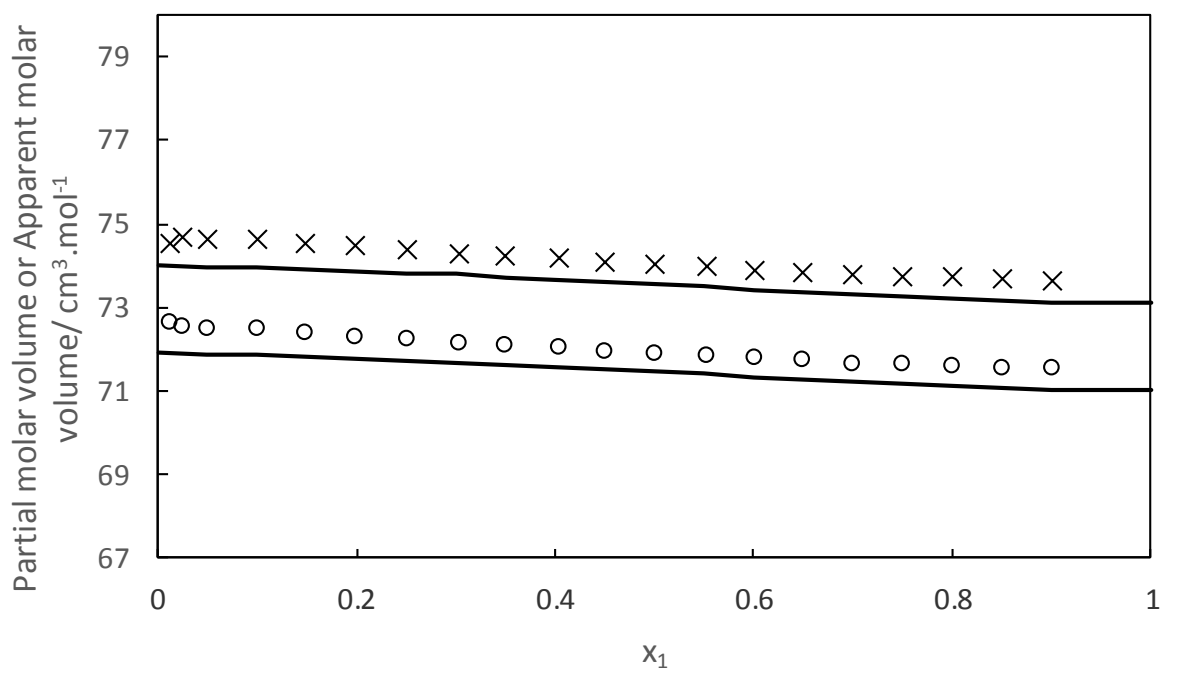

Figure 10: Calculated partial molar volume using Redlich-Kister correlation (solid line) and experimental apparent molar volume (symbols) of furan(1) + 1-Octanol(2) system using RedlichKister correlation as a function of furan mole fraction at atmospheric pressure and 2 different temperatures: (O) $283.15 \mathrm{~K},(x) 303.15 \mathrm{~K}$.

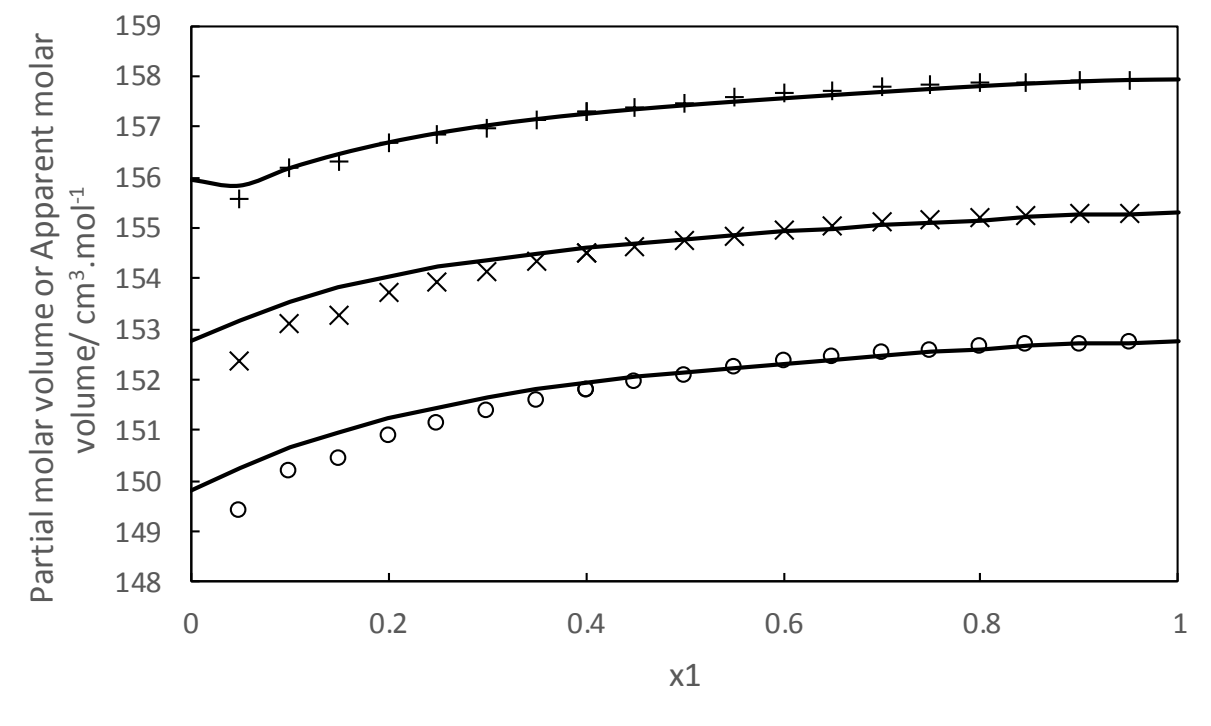

Figure 11: Calculated partial molar volume using Redlich-Kister correlation (solid line) and experimental apparent molar volume (symbols) of eugenol(1) + 1-Octanol(2) system using RedlichKister correlation as a function of eugenol mole fraction at atmospheric pressure and 2 different temperatures: (o) $283.15 \mathrm{~K},(\times) 303.15 \mathrm{~K},(+) 323.15 \mathrm{~K}$. 


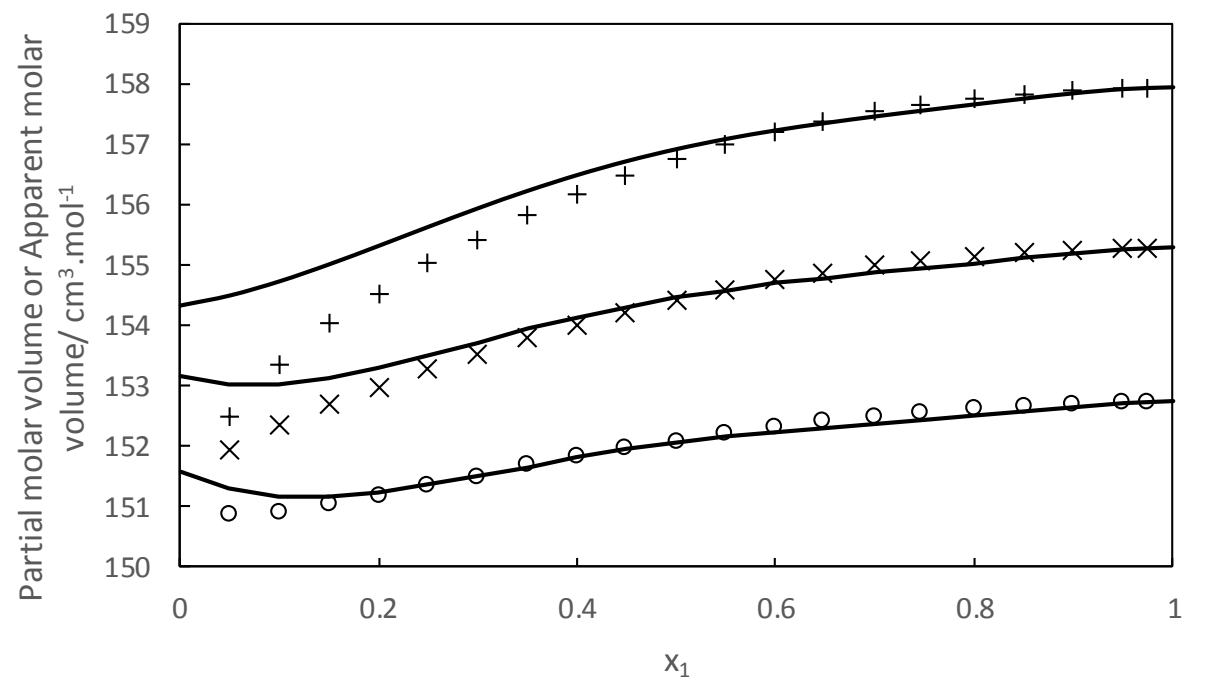

Figure 12: Calculated partial molar volume using Redlich-Kister correlation (solid line) and experimental apparent molar volume (symbols) of eugenol(1) + n-hexane(2) system using RedlichKister correlation as a function of eugenol mole fraction at atmospheric pressure and 2 different temperatures: (o) $283.15 \mathrm{~K},(\times) 303.15 \mathrm{~K},(+) 323.15 \mathrm{~K}$. 


\subsection{Prigogine-Flory-Patterson model}

The Prigogine Flory Patterson model (PFP) [8] was developed to analyze excess thermodynamic properties of polar mixtures (Gepert et al. [9], Galvao and Francesconi [10], Torres et al. [11]). On the same idea, in order to predict excess volumes, several authors [12] use the Flory equation [13] while others [14], [15] use the Prigogine-Flory-Patterson (PFP) theory, which is the Flory equation combined with the Prigogine corresponding states principle.

The equation of state used in PFP model is determined for pure liquid and mixtures. It is written using reduced quantities (Eq. 15).

$$
\frac{\tilde{p} \tilde{v}}{\tilde{T}}=\frac{\tilde{v}^{1 / 3}}{\tilde{v}^{1 / 3}-1}-\frac{1}{\tilde{v} \tilde{T}}
$$

With $\tilde{p}=\frac{p}{p^{*}}, \tilde{v}=\frac{v}{v^{*}}$ and $\tilde{T}=\frac{T}{T^{*}} \cdot T^{*}, p^{*}$ and $v^{*}$ are the characteristics parameters obtained directly from the thermodynamic properties of the pure liquid.

Eq. 16 presents the expression of the excess volume based on PFP theory. The PFP model describes the interaction between the chemical species. The physical interaction is the sum of 3 contributions:

$$
\begin{aligned}
\frac{v^{E}}{x_{1} v_{1}^{*}+x_{2} v_{2}^{*}}=\tilde{T} & \frac{\partial \tilde{v}}{\partial \tilde{T}} \psi_{1} \theta_{2} \frac{\chi_{12}}{P_{1}^{*}} \\
& -\frac{1}{2}\left(\frac{\tilde{v}_{1}-\tilde{v}_{2}}{\frac{\partial \tilde{v}}{\partial \tilde{T}}}\right)^{2} \frac{\partial^{2} \tilde{v}}{\partial \tilde{T}^{2}} \psi_{1} \psi_{2}+\frac{\left(\tilde{v}_{1}-\tilde{v}_{2}\right)\left(P_{1}^{*}-P_{2}^{*}\right)}{P_{2}^{*} \psi_{1}+P_{1}^{*} \psi_{2}}
\end{aligned}
$$

i. Interactional contribution. This contribution is proportional to the Flory parameter $\chi_{12}$. Flory parameter is also called cross parameter. It reflects the energy change during the formation of contacts between the unlike molecules. The $\chi_{12}$ parameter can be calculated using solubility parameter defined by Hildebrand: $\chi_{12}=\frac{v\left(\delta_{1}-\delta_{2}\right)^{2}}{R T}+\beta(\beta$ is an empirical constant equal to 0.34). This parameter can be also adjusted on experimental data. Volume $v$ is the molar volume of the solvent. At the origin, the model 
was developed to study solubility of polymer in solvent. In our application, is it judicious to consider this molar volume independent of the composition?

ii. Curvature contribution or free volume contribution (link to configurational entropy). This contribution is due to the difference in the degree of thermal expansion between the molecules.

iii. P* contribution. This contribution takes into account the difference of internal pressure $\left(\pi=\left(\frac{\partial U}{\partial v}\right)_{T}\right)$ and reduced volume of components.

Here the tilde refers to an average reduced quantity, for example $\widetilde{v}=\psi_{1} \tilde{v}_{1}+\psi_{2} \tilde{v}_{2}$ with $\tilde{v}_{i}=\left(\frac{1+\frac{4}{3} \alpha_{i} T}{1+\alpha_{i} T}\right)^{3}$ and $\alpha_{i}$ is the thermal expansion coefficient. Reduced temperature is obtained through PFP model by $\tilde{T}=\frac{\tilde{v}^{1 / 3}-1}{\tilde{v}^{4 / 3}}$ and $\tilde{V}^{1 / 3}-1=\frac{\alpha T / 3}{1+\alpha T}$.

The characteristic pressure is given by $P_{i}^{*}=\frac{\alpha_{i}}{\kappa_{T i}} T \tilde{v}_{i}^{2}$. Although, several authors consider $\alpha / \mathrm{k}$ as an adjustable parameter, the thermal expansion coefficient is $\alpha=\frac{1}{v}\left(\frac{\partial v}{\partial T}\right)_{P}$ and the isothermal compressibility is $\kappa_{T}=-\frac{1}{v}\left(\frac{\partial v}{\partial P}\right)_{T}$

The contact energy fraction is $\psi_{1}=1-\psi_{2}=\frac{\phi_{1} P_{1}^{*}}{\phi_{1} P_{1}^{*}+\phi_{2} P_{2}^{*}}$, the hardcore volume fraction is $\phi_{1}=1-\phi_{2}=\frac{x_{1} v_{1}^{*}}{x_{1} v_{1}^{*}+x_{2} v_{2}^{*}}$ and the surface fraction is $\theta_{2}=\frac{\phi_{2} s_{2}}{\phi_{1} s_{1}+\phi_{2} s_{2}}$.

$s_{i}$ being the molecular surface/volume ratios and $s_{1} / s_{2}$ can be approximated by $\left(v_{2}^{*} / v_{1}^{*}\right)^{1 / 3}$, the surface fraction is also expressed by: $\theta_{2}=\frac{1}{1+\frac{\phi_{1}}{\phi_{2}}\left(\frac{v_{2}^{*}}{v_{1}^{*}}\right)^{1 / 3}}$.

Consequently, using the PFP equation of state and the different defined variables, the expression of the excess volume can be determined (Eq. 17). 


$$
\begin{gathered}
\frac{v^{E}}{x_{1} v_{1}^{*}+x_{2} v_{2}^{*}}=\frac{\left(\tilde{v}^{1 / 3}-1\right) \tilde{v}^{2 / 3}}{4 / 3 \tilde{v}^{-1 / 3}-1} \psi_{1} \theta_{2} \frac{\chi_{12}}{P_{1}^{*}}-\frac{\left(\tilde{v}_{1}-\tilde{v}_{2}\right)^{2}\left(14 / 9 \tilde{v}^{-1 / 3}-1\right)}{\left(4 / 3 \tilde{v}^{-1 / 3}-1\right) \tilde{v}} \psi_{1} \psi_{2} \\
+\frac{\left(\tilde{v}_{1}-\tilde{v}_{2}\right)\left(P_{1}^{*}-P_{2}^{*}\right)}{P_{2}^{*} \psi_{1}+P_{1}^{*} \psi_{2}} \psi_{1} \psi_{2}
\end{gathered}
$$

Unity of $v^{E}$ is imposed by the choice of unity of $v_{i}^{*}$ and $v_{i}$. Temperature is in Kelvin. The choice of unity of pressure (among other $\mathrm{P}_{\mathrm{i}}^{*}$ ) imposed the unity of $\chi_{12}$.

\section{a. PFP Parametrization}

For a pure component, the isothermal compressibility $\mathrm{K}_{\mathrm{Ti}}$ is related to the isentropic compressibility $\kappa_{\mathrm{S}}$ via the Maxwell's relations (Eq. 18).:

$$
\kappa_{T i}=\kappa_{S}+\alpha^{2} v_{i}^{0} \frac{T}{C_{p}}
$$

where $v_{i}\left[\mathrm{~m}^{3} / \mathrm{mol}\right]$ is the molar volume of the compound $i, \kappa_{S}$ is the isentropic compressibility $\kappa_{S}=-\frac{1}{v}\left(\frac{\partial v}{\partial P}\right)_{S}\left[\mathrm{~Pa}^{-1}\right]$ and is known thanks to the measurements of speed of sound $\mathrm{c}_{\text {liquid }}[\mathrm{m} / \mathrm{s}]$ and density $\rho\left[\mathrm{kg} / \mathrm{m}^{3}\right]$ reached in this work. Indeed, these three properties are linked in the liquid phase via: $c_{\text {liquid }}=\sqrt{\frac{1}{\kappa_{S} \rho}}$. In order to estimate PFP parameters, densities and speed of sound data for furan, ethanol, n-hexane, Eugenol and 1-Octanol were used. Table 11 presents the speed of sound data for the concerned chemicals.

Concerning liquid heat capacity $C_{p}\left[J \cdot \mathrm{mol}^{-1} \cdot \mathrm{K}^{-1}\right]$, we have used the data predicted by REFPROP [16] for ethanol, the data from Guthrie et al. [17] for furan, the data from Karabaev et al. [18] for eugenol, the data from Melnikov et al. [19] for the $n$-hexane and the data from Rubini et al.[20] for the 1Octanol. 
Table 11: Speed of sound data at atmospheric pressure $\left(U_{p}= \pm 0.03 \mathrm{kPa}, U_{T}= \pm 0.01 K, U_{c}= \pm 0.5 \mathrm{~m} . \mathrm{s}^{-1}(\mathrm{k}=2)\right)$.

\begin{tabular}{|c|c|c|c|c|c|c|c|c|c|}
\hline \multicolumn{2}{|c|}{ Eugenol } & \multicolumn{2}{|c|}{ Furan } & \multicolumn{2}{|c|}{ Ethanol } & \multicolumn{2}{|c|}{ 1-Octanol } & \multicolumn{2}{|c|}{ n-hexane } \\
\hline $\mathbf{T} / \mathbf{K}$ & c/ m.s. & $\mathbf{T} / \mathbf{K}$ & $\mathrm{c} / \mathbf{m}^{-\mathbf{s}^{-1}}$ & $\mathbf{T} / \mathbf{K}$ & $\mathrm{c} / \mathrm{m}^{\mathrm{s}} \mathrm{s}^{-1}$ & $\mathbf{T} / \mathrm{K}$ & $\mathrm{c} / \mathrm{m}^{-\mathrm{s}^{-1}}$ & $\mathbf{T} / \mathbf{K}$ & $\mathrm{c} / \mathrm{m}^{-\mathrm{s}^{-1}}$ \\
\hline 273.15 & 1569.7 & 278.16 & 1229.40 & 278.16 & 1212.2 & 278.16 & 1416.4 & 283.15 & 1145.3 \\
\hline 275.15 & 1562.0 & 279.14 & 1224.70 & 279.14 & 1208.8 & 279.15 & 1413.2 & 288.15 & 1123.0 \\
\hline 277.16 & 1554.4 & 280.15 & 1219.80 & 280.14 & 1205.3 & 280.15 & 1409.7 & 293.14 & 1100.4 \\
\hline 279.15 & 1548.1 & 281.15 & 1214.80 & 281.14 & 1201.8 & 281.15 & 1406.2 & 298.14 & 1077.7 \\
\hline 281.15 & 1540.6 & 282.15 & 1209.90 & 282.14 & 1198.3 & 282.15 & 1402.7 & 303.15 & 1055.2 \\
\hline 283.15 & 1533.1 & 283.15 & 1204.90 & 283.14 & 1194.8 & 283.15 & 1399.3 & 308.15 & 1032.7 \\
\hline 285.15 & 1525.7 & 284.15 & 1199.90 & 284.14 & 1191.3 & 284.15 & 1395.8 & 313.15 & 1010.4 \\
\hline 287.15 & 1518.2 & 285.14 & 1195.00 & 285.14 & 1187.8 & 285.15 & 1392.3 & 318.15 & 988.1 \\
\hline 289.15 & 1510.5 & 286.14 & 1190.00 & 286.14 & 1184.3 & 286.15 & 1388.8 & 323.14 & 965.8 \\
\hline 291.15 & 1503.1 & 287.14 & 1185.10 & 287.15 & 1180.8 & 287.15 & 1385.5 & & \\
\hline 293.15 & 1496.1 & 288.14 & 1180.10 & 288.15 & 1177.3 & 288.15 & 1382.2 & & \\
\hline 295.15 & 1488.6 & 289.14 & 1175.20 & 289.15 & 1173.9 & 289.15 & 1379.1 & & \\
\hline 297.15 & 1481.3 & 290.14 & 1170.20 & 290.15 & 1170.4 & 290.15 & 1375.3 & & \\
\hline 299.15 & 1474.0 & 291.14 & 1165.30 & 291.15 & 1166.9 & 291.15 & 1371.7 & & \\
\hline 301.16 & 1466.7 & 292.14 & 1160.40 & 292.15 & 1163.5 & 292.15 & 1368.2 & & \\
\hline 303.16 & 1459.5 & 293.14 & 1155.40 & 293.15 & 1160.1 & 293.15 & 1364.7 & & \\
\hline 305.16 & 1451.6 & 294.15 & 1150.50 & 294.15 & 1156.7 & 294.15 & 1361.2 & & \\
\hline 307.16 & 1444.3 & 295.15 & 1145.60 & 295.15 & 1153.2 & 295.15 & 1357.7 & & \\
\hline 309.16 & 1437.1 & 296.15 & 1140.70 & 296.15 & 1149.8 & 296.15 & 1354.3 & & \\
\hline 311.16 & 1429.8 & 297.15 & 1135.80 & 297.15 & 1146.4 & 297.15 & 1350.8 & & \\
\hline 313.16 & 1422.6 & 298.15 & 1130.90 & 298.15 & 1143.0 & 298.15 & 1347.4 & & \\
\hline 315.16 & 1415.3 & 299.15 & 1126.00 & 299.15 & 1139.6 & 299.15 & 1343.9 & & \\
\hline 317.16 & 1408.1 & 300.15 & 1121.10 & 300.15 & 1136.2 & 300.15 & 1340.5 & & \\
\hline 319.16 & 1400.9 & 301.15 & 1116.20 & 301.15 & 1132.8 & 301.15 & 1337.1 & & \\
\hline 321.16 & 1393.7 & 302.15 & 1111.30 & 302.15 & 1129.4 & 302.15 & 1333.7 & & \\
\hline 323.16 & 1386.6 & 303.15 & 1106.40 & 303.15 & 1126.0 & 303.15 & 1330.3 & & \\
\hline 325.16 & 1379.4 & 304.15 & 1101.50 & 304.15 & 1122.6 & 304.15 & 1326.9 & & \\
\hline 327.16 & 1372.3 & & & & & & & & \\
\hline
\end{tabular}


$329.16 \quad 1365.2$

$331.16 \quad 1358.1$

$335.15 \quad 1344.0$

$337.15 \quad 1336.9$

$339.15 \quad 1329.9$

$341.15 \quad 1322.9$

$343.15 \quad 1315.9$ 
In order to calculate the molar volume, the thermal expansion, the isentropic compressibility and the isothermal compressibility, we have used the previous thermodynamic relations and considered in the concerned range of temperature, at atmospheric pressure that the mathematical relation for the different thermodynamic quantities were expressed by a second order polynomial expression with temperature (Eq. 19).

$$
F(T)=a T^{2}+b T+c
$$

The adjusted parameters $a, b$ and $c$ are presented in tables 12-16 for the different chemicals. Instead of providing the deviation with experimental data, we have indicated the standard deviation of each fitted parameter.

Table 12: Values of the second order polynomial expression (Eq. 13) for 1-Octanol

\begin{tabular}{ccccccc}
\hline Property/F & $\mathbf{a}$ & $\mathbf{u}(\mathbf{a})$ & $\mathbf{b}$ & $\mathbf{u}(\mathbf{b})$ & $\mathbf{c}$ & $\mathbf{u}(\mathbf{c})$ \\
\hline $\mathrm{v} / \mathrm{cm}^{3} \cdot \mathrm{mol}^{-1}$ & $1.70 \mathrm{E}-04$ & $2 \mathrm{E}-06$ & 0.032 & 0.001 & 133.8 & 0.2 \\
$\alpha / \mathrm{K}^{-1}$ & $-2.12 \mathrm{E}-09$ & $1 \mathrm{E}-11$ & $2.695 \mathrm{E}-06$ & $6 \mathrm{E}-09$ & $2.3 \mathrm{E}-04$ & $9 \mathrm{E}-07$ \\
$\mathrm{Cp} / \mathrm{J} \cdot \mathrm{mol}^{-1} \cdot \mathrm{K}^{-1}$ & & & 1.07 & 0.03 & -14.51 & 9 \\
$\chi_{S} / \mathrm{Pa}^{-1}$ & $1.56 \mathrm{E}-14$ & $4 \mathrm{E}-16$ & $-5.3 \mathrm{E}-12$ & $2 \mathrm{E}-13$ & $8.7 \mathrm{E}-10$ & $3 \mathrm{E}-11$ \\
$\chi_{T} / \mathrm{Pa}^{-1}$ & $1.5822 \mathrm{E}-14$ & $1 \mathrm{E}-18$ & $-4.9808 \mathrm{E}-12$ & $7 \mathrm{E}-16$ & $8.594 \mathrm{E}-10$ & $1 \mathrm{E}-13$ \\
\hline
\end{tabular}

Table 13: Values of the PFP parameters for $n$-hexane

\begin{tabular}{ccccccc}
\hline Property/F & $\mathbf{a}$ & $\mathbf{u}(\mathbf{a})$ & $\mathbf{b}$ & $\mathbf{u}(\mathbf{b})$ & $\mathbf{c}$ & $\mathbf{u}(\mathbf{c})$ \\
\hline $\mathrm{v} / \mathrm{cm}^{3} \cdot \mathrm{mol}^{-1}$ & $4.29 \mathrm{E}-04$ & $1 \mathrm{E}-05$ & -0.0730 & $6 \mathrm{E}-03$ & 115.1 & $9 \mathrm{E}-01$ \\
$\alpha / \mathrm{K}^{-1}$ & $-1.090 \mathrm{E}-08$ & $1 \mathrm{E}-10$ & $1.109 \mathrm{E}-05$ & $6 \mathrm{E}-08$ & $-9.48 \mathrm{E}-04$ & $9 \mathrm{E}-06$ \\
$\mathrm{Cp} / \mathrm{J} \cdot \mathrm{mol}^{-1} \cdot \mathrm{K}^{-1}$ & $1.45 \mathrm{E}-03$ & $9 \mathrm{E}-05$ & $-4.93 \mathrm{E}-01$ & $6 \mathrm{E}-02$ & 213.40 & 9 \\
$\chi_{S} / \mathrm{Pa}^{-1}$ & $7.45 \mathrm{E}-14$ & $7 \mathrm{E}-16$ & $-3.15 \mathrm{E}-11$ & $4 \mathrm{E}-13$ & $4.09 \mathrm{E}-09$ & $6 \mathrm{E}-11$ \\
$\chi_{T} / \mathrm{Pa}^{-1}$ & $7.685 \mathrm{E}-14$ & $6 \mathrm{E}-17$ & $-2.929 \mathrm{E}-11$ & $4 \mathrm{E}-14$ & $3.604 \mathrm{E}-09$ & $5 \mathrm{E}-12$ \\
\hline
\end{tabular}

Table 14: Values of the PFP parameters for eugenol

\begin{tabular}{ccccccc}
\hline Property/F & $\mathbf{a}$ & $\mathbf{u}(\mathbf{a})$ & $\mathbf{b}$ & $\mathbf{u}(\mathbf{b})$ & $\mathbf{c}$ & $\mathbf{u}(\mathbf{c})$ \\
\hline $\mathrm{v} / \mathrm{cm}^{3} \cdot \mathrm{mol}^{-1}$ & $1.15 \mathrm{E}-04$ & $5 \mathrm{E}-07$ & 0.0601 & $3 \mathrm{E}-04$ & 126.49 & $5 \mathrm{E}-02$ \\
$\alpha / \mathrm{K}^{-1}$ & $-1.267 \mathrm{E}-09$ & $2 \mathrm{E}-12$ & $1.551 \mathrm{E}-06$ & $1 \mathrm{E}-09$ & $4.830 \mathrm{E}-04$ & $2 \mathrm{E}-07$ \\
$\mathrm{Cp} / \mathrm{J} \cdot \mathrm{mol}^{-1} \cdot \mathrm{K}^{-1}$ & & & $1.42 \mathrm{E}+00$ & $4 \mathrm{E}-02$ & -74.59 & 9 \\
$\chi_{S} / \mathrm{Pa}^{-1}$ & $1 \mathrm{E}-14$ & $3 \mathrm{E}-17$ & $-3.48 \mathrm{E}-12$ & $2 \mathrm{E}-14$ & $5.75 \mathrm{E}-10$ & $3 \mathrm{E}-12$ \\
$\chi_{T} / \mathrm{Pa}^{-1}$ & $1.01237 \mathrm{E}-14$ & $9 \mathrm{E}-19$ & $-3.3423 \mathrm{E}-12$ & $6 \mathrm{E}-16$ & $6.1855 \mathrm{E}-10$ & $9 \mathrm{E}-14$ \\
\hline
\end{tabular}


Table 15: Values of the PFP parameters for Ethanol

\begin{tabular}{ccccccc}
\hline Property/F & $\mathbf{a}$ & $\mathbf{u}(\mathbf{a})$ & $\mathbf{b}$ & $\mathbf{u}(\mathbf{b})$ & $\mathbf{c}$ & $\mathbf{u}(\mathbf{c})$ \\
\hline $\mathrm{v} / \mathrm{cm}^{3} \cdot \mathrm{mol}^{-1}$ & $9.85 \mathrm{E}-05$ & $9 \mathrm{E}-07$ & 0.006 & 0.001 & 48.3 & 0.1 \\
$\alpha / \mathrm{K}^{-1}$ & $-4.22 \mathrm{E}-09$ & $2 \mathrm{E}-11$ & $4.67 \mathrm{E}-06$ & $1 \mathrm{E}-08$ & $7.8 \mathrm{E}-05$ & 8.8 \\
$\mathrm{Cp} / \mathrm{J} \cdot \mathrm{mol}^{-1} \cdot \mathrm{K}^{-1}$ & & & 0.35 & 0.00 & 0.7 \\
$\chi_{S} / \mathrm{Pa}^{-1}$ & $2.70 \mathrm{E}-14$ & $2 \mathrm{E}-16$ & $-9.2 \mathrm{E}-12$ & $1 \mathrm{E}-13$ & $1.32 \mathrm{E}-09$ & $2 \mathrm{E}-11$ \\
$\chi_{T} / \mathrm{Pa}^{-1}$ & $2.7408 \mathrm{E}-14$ & $4 \mathrm{E}-18$ & $-8.473 \mathrm{E}-12$ & $2 \mathrm{E}-15$ & $1.2527 \mathrm{E}-09$ & $4 \mathrm{E}-13$ \\
\hline
\end{tabular}

Table 16: Values of the PFP parameters for Furan

\begin{tabular}{ccccccc}
\hline Property/F & $\mathbf{a}$ & $\mathbf{u}(\mathbf{a})$ & $\mathbf{b}$ & $\mathbf{u}(\mathbf{b})$ & $\mathbf{c}$ & $\mathbf{u}(\mathbf{c})$ \\
\hline $\mathrm{v} / \mathrm{cm}^{3} \cdot \mathrm{mol}^{-1}$ & $2.47 \mathrm{E}-04$ & $1 \mathrm{E}-06$ & -0.0388 & $8 \mathrm{E}-04$ & 62.7 & 0.1 \\
$\alpha / \mathrm{K}^{-1}$ & $-1.185 \mathrm{E}-08$ & $3 \mathrm{E}-11$ & $1.162 \mathrm{E}-05$ & $2 \mathrm{E}-08$ & $-9.24 \mathrm{E}-04$ & $2 \mathrm{E}-06$ \\
$\mathrm{Cp} / \mathrm{J} \cdot \mathrm{mol}^{-1} \cdot \mathrm{K}^{-1}$ & $6.2 \mathrm{E}-04$ & $2 \mathrm{E}-04$ & $-1.59 \mathrm{E}-01$ & 0.1 & 107.03 & 20 \\
$\chi_{S} / \mathrm{Pa}^{-1}$ & $5.20 \mathrm{E}-14$ & $3 \mathrm{E}-16$ & $-2.25 \mathrm{E}-11$ & $1 \mathrm{E}-13$ & $2.92 \mathrm{E}-09$ & $2 \mathrm{E}-11$ \\
$\chi_{T} / \mathrm{Pa}^{-1}$ & $5.473 \mathrm{E}-14$ & $5 \mathrm{E}-17$ & $-2.032 \mathrm{E}-11$ & $3 \mathrm{E}-14$ & $2.452 \mathrm{E}-09$ & $5 \mathrm{E}-12$ \\
\hline
\end{tabular}

\section{b. Results and Discussion}

We have applied the PFP model for the 4 binary systems at 283, 293 and 303K. In order to adjust the $\chi_{12}$ parameter, we have minimized the objective function (Eq. 20) using PFP parameters calculated using Eq. 19 for each pure component property.

$O F=\sum_{i}\left(v_{\mathrm{exp}, i}^{E}-v_{P F P, i}^{E}\right)^{2}$

Tables 17-19 presents the values of the parameters of each pure component at 283, 293 and $298 \mathrm{~K}$. The values of $\chi_{12}$ parameters are presented in Table 20. This parameter is at the origin independent of the composition (Hansen [21]) but it was used to represent the volumetric properties of binary systems involving polymer and solvent. $\chi_{12}$ depends on molar volume of the solvent but our case of study considers the specie as the solvent, particularly if the molar composition is close to 0.5 . So it can be not judicious to consider that $\chi_{12}$ parameter is independent of volume fraction. In 1984, Mulder and Smolders [22] have mentioned that excess functions are strongly concentration dependent. They have indicated that $\chi_{12}$ parameter (a free energy parameter) can be expressed from the Excess Gibbs energy of mixing. They have studied the ethanol water binary system and show the evolution of $\chi_{12}$ as a function of volume fractions. Also, Hansen [21] has shown that the $\chi_{12}$ parameter, originally calculated using Hildebrand solubility parameter, can be calculated by a $\chi_{12}$ parameter which takes into account dispersive, polar and hydrogen bonding interactions. In consequence, we have decided to modify the cross parameter $\chi_{12}$ by using Eq. 21 . Results are presented in Table 20. 
$\chi_{12}=\chi_{12}^{0}\left(a+b\left(\phi_{1}-\varphi_{2}\right)\right)$

The $\chi_{12}^{0}$ parameter is determined by considering no composition dependency. a and $\mathrm{b}$ parameters are adjusted using objective function defined by equation 20 and taking $\chi_{12}^{0}$ as a constant.

Table 17: Physical constants of pure compounds at $283.15 \mathrm{~K}$, used for calculations of Excess molar volume $v^{E}$ with PFP model: $v_{i}^{\circ}$ : molar volume, $\alpha_{i}$ coefficient of thermal expansion $\chi_{T}$ Isothermal compressibility, Reduction parameters of volume $\mathbf{v}_{\mathrm{i}}{ }^{*}$ and Pressure $\mathrm{P}_{\mathrm{i}}{ }^{*}$.

\begin{tabular}{cccccc}
\hline Compound & $\mathbf{v}_{\mathbf{i}}{ }^{\circ} / \mathbf{c m}^{\mathbf{3}} \cdot \mathbf{m o l}^{-1}$ & $\mathbf{1 0}^{\mathbf{3}} \boldsymbol{\alpha}_{\mathbf{i}} / \mathbf{K}^{\mathbf{- 1}}$ & $\mathbf{1 0}^{\mathbf{3}} \chi_{\mathbf{T}} / \mathbf{M P a}^{-1}$ & $\mathbf{v}_{\mathbf{i}}^{*} / \mathbf{c m}^{3} \cdot \mathbf{m o l}^{-1}$ & $\mathbf{P}_{\mathbf{i}}^{*} / \mathbf{M P a}$ \\
\hline Eugenol & 152.728 & 0.818 & 0.484 & 127.280 & 689.143 \\
Furan & 72.535 & 1.460 & 1.196 & 54.876 & 603.583 \\
n-hexane & 128.814 & 1.319 & 1.470 & 99.309 & 427.080 \\
n-1-Octanol & 156.514 & 0.822 & 0.717 & 130.344 & 467.630 \\
Ethanol & 57.727 & 1.064 & 1.050 & 46.191 & 447.981 \\
\hline
\end{tabular}

Table 18: Physical constants of pure compounds at 293.15 K, used for calculations of Excess molar volume $v^{E}$ with PFP model: $v_{i}^{\circ}$ : molar volume, $\alpha_{i}$ coefficient of thermal expansion $\chi_{T}$ Isothermal compressibility, Reduction parameters of volume $v_{i}{ }^{*}$ and Pressure $P_{i}{ }^{*}$.

\begin{tabular}{cccccc}
\hline Compound & $\mathbf{v}_{\mathbf{i}}{ }^{\circ} / \mathbf{~ c m}^{\mathbf{3}} \cdot \mathbf{m o l}^{-1}$ & $\mathbf{1 0}^{\mathbf{3}} \boldsymbol{\alpha}_{\mathbf{i}} / \mathbf{K}^{-\mathbf{1}}$ & $\mathbf{1 0}^{\mathbf{3}} \chi_{\mathrm{T}} / \mathbf{M P a}^{-\mathbf{1}}$ & $\mathbf{v}_{\mathbf{i}}{ }^{*} / \mathbf{c m}^{\mathbf{3}} \cdot \mathbf{m o l}^{\mathbf{1}}$ & $\mathbf{P}_{\mathrm{i}}^{*} / \mathbf{M P a}$ \\
\hline Eugenol & 153.993 & 0.826 & 0.508 & 127.506 & 694.676 \\
Furan & 72.535 & 1.460 & 1.196 & 54.516 & 633.197 \\
n-hexane & 130.555 & 1.367 & 1.619 & 99.348 & 427.224 \\
n-1-Octanol & 157.815 & 0.837 & 0.758 & 130.421 & 473.226 \\
Ethanol & 58.350 & 1.086 & 1.123 & 46.261 & 450.833 \\
\hline
\end{tabular}


Table 19: Physical constants of pure compounds at $298.15 \mathrm{~K}$, used for calculations of Excess molar volume $v^{\mathrm{E}}$ with PFP model: $v_{i}^{\circ}:$ molar volume, $\alpha_{i}$ coefficient of thermal expansion $\chi_{T}$ Isothermal compressibility, Reduction parameters of volume $v_{i}{ }^{*}$ and Pressure $P_{i}^{*}$.

\begin{tabular}{cccccc}
\hline Compound & $\mathbf{v}_{\mathbf{i}}{ }^{\circ} / \mathbf{~ c m}^{\mathbf{3}} \cdot \mathbf{m o l}^{-1}$ & $\mathbf{1 0}^{3} \boldsymbol{\alpha}_{\mathbf{i}} / \mathbf{K}^{-\mathbf{1}}$ & $\mathbf{1 0}^{3} \chi_{\mathbf{T}} / \mathbf{M P a}^{-\mathbf{1}}$ & $\mathbf{v}_{\mathbf{i}}{ }^{*} / \mathbf{c m}^{3} \cdot \mathbf{m o l}^{-\mathbf{1}}$ & $\mathbf{P}_{\mathbf{i}}^{*} / \mathbf{M P a}$ \\
\hline Eugenol & 154.634 & 0.830 & 0.522 & 127.627 & 695.877 \\
Furan & 73.073 & 1.483 & 1.257 & 54.575 & 630.661 \\
n-hexane & 131.458 & 1.390 & 1.700 & 99.408 & 426.011 \\
n-1-Octanol & 158.478 & 0.844 & 0.780 & 130.468 & 475.530 \\
Ethanol & 58.669 & 1.097 & 1.162 & 46.300 & 451.726 \\
\hline
\end{tabular}

Table 20: $\chi_{12}$ parameters used in the PFP theory

\begin{tabular}{ccccccccccc}
\hline \multirow{2}{*}{ System } & \multicolumn{3}{c}{$\mathbf{2 8 3 . 1 5} \mathbf{K}$} & \multicolumn{3}{c}{$\mathbf{2 9 3 . 1 5 ~ K}$} & \multicolumn{3}{c}{$\mathbf{2 9 8 . 1 5 ~ K}$} \\
\cline { 2 - 10 } & $\chi_{12}^{0}$ & $\mathbf{a}$ & $\mathbf{b}$ & $\chi_{12}^{0}$ & $\mathbf{a}$ & $\mathbf{b}$ & $\chi_{12}^{0}$ & $\mathbf{a}$ & $\mathbf{b}$ \\
\hline Furan + Ethanol & -33.97 & 1.0266 & -0.4704 & -30.14 & 1.0282 & -0.5375 & -27.98 & 1.034 & -0.5817 \\
Furan + 1-Octanol & 12.39 & 0.9051 & -0.4545 & 5.68 & 1.0761 & -0.5300 & 2.14 & 1.0761 & -0.5300 \\
Eugenol + 1-Octanol & -38.82 & 1.0161 & 0.1728 & -33.09 & 1.0181 & 0.1936 & -30.38 & 1.0187 & 0.2003 \\
Eugenol + n-hexane & 59.25 & 0.9872 & -0.2000 & 62.36 & 0.9884 & -0.1667 & 63.43 & 0.9892 & -0.1501 \\
\hline
\end{tabular}

Figs. 13-16 show the comparison between the two treatments with or without composition dependency of $\chi_{12}$ parameter at $293.15 \mathrm{~K}$. Figs. S6-S9 (Supplementary information) show the results obtained at 298K. Figs. S10-S13 (Supplementary information) show the results obtained at 283K. As we can see, we have a better correlation of the data when the $\chi_{12}$ parameter is dependent of the composition. We have also evaluated the contribution of the different contribution of PFP model to excess volume at $293.15 \mathrm{~K}$. For the 4 systems, the results are presented in Tables 21- 24. Tables S2-S5 (Supplementary information) show the results obtained at 298K. Tables S5-S9 (Supplementary information) show the results obtained at 283K. For the system furan ethanol, the main contribution to excess volume is attributed to the interactional contribution. It highlights the importance of the $\chi_{12}$ parameter in the data treatment and the dependence to the composition of this parameter. It highlights the effect of polar and $\mathrm{H}$-bonding interactions certainly linked to the composition of the mixture. For furan 1-Octanol, all the different types of interaction contribute at the same level to excess volume. For Eugenol 1-Octanol, the main contribution to excess volume is attributed to the interactional contribution. There is no effect of free volume. For the last binary system, eugenol nhexane, it seems that the main contribution to excess volume are free volume effect and internal 
pressure effect. On Figs. 17-18, we can see the most relevant contribution for the furan + ethanol and eugenol $+n$-hexane binary systems to excess molar volume. Figure 19 shows the evolution of $\chi_{12}$ parameters with temperature. We can observe for each case a linear trend.

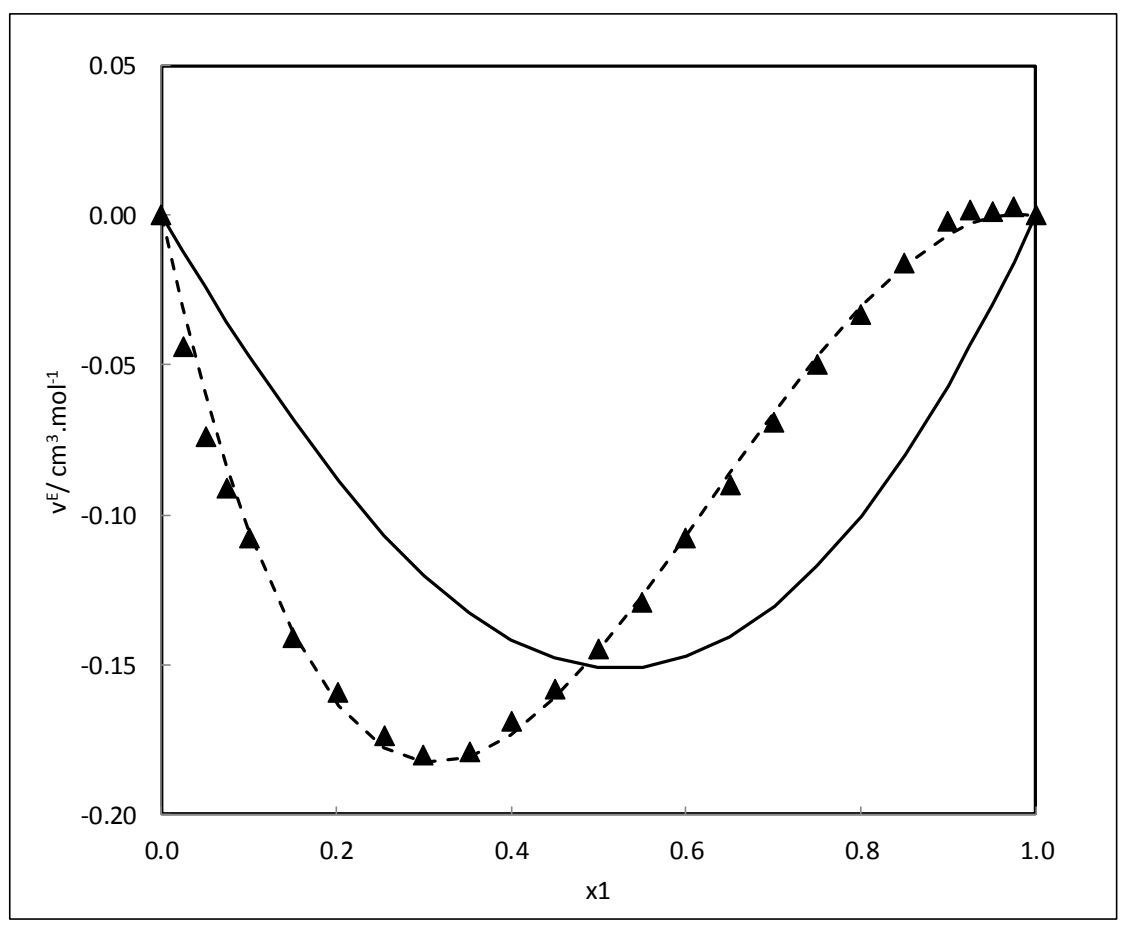

Figure 13 : Excess volumes for the furan(1) + Ethanol(2) binary system at $293.15 \mathrm{~K}$ as a function of molar composition. Symbols: experimental data, solid line: Calculated using PFP model with constant value of $\chi_{12}$, dashed line: Calculated with PFP model with constant $\chi_{12}$ composition dependent.

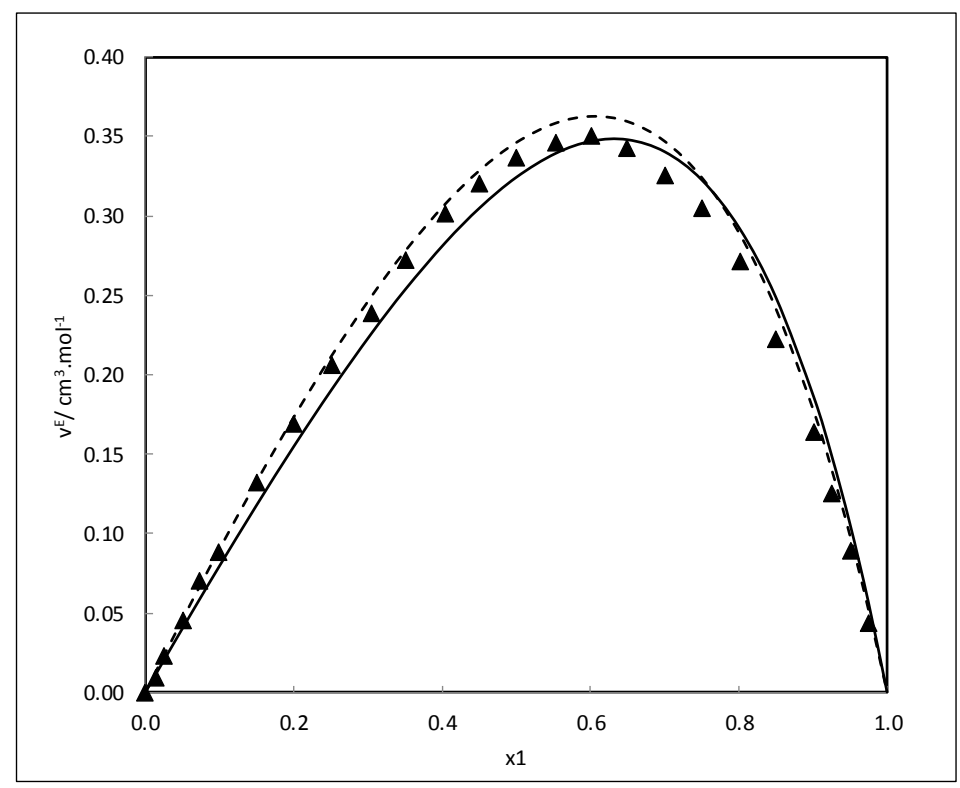

Figure 14 : Excess volumes for the Furan(1) + 1-Octanol(2) binary system at $293.15 \mathrm{~K}$ as a function of molar composition. Symbols: experimental data, solid line: Calculated using PFP model with constant value of $\chi_{12}$, dashed line: Calculated with PFP model with constant $\chi_{12}$ composition dependent. 


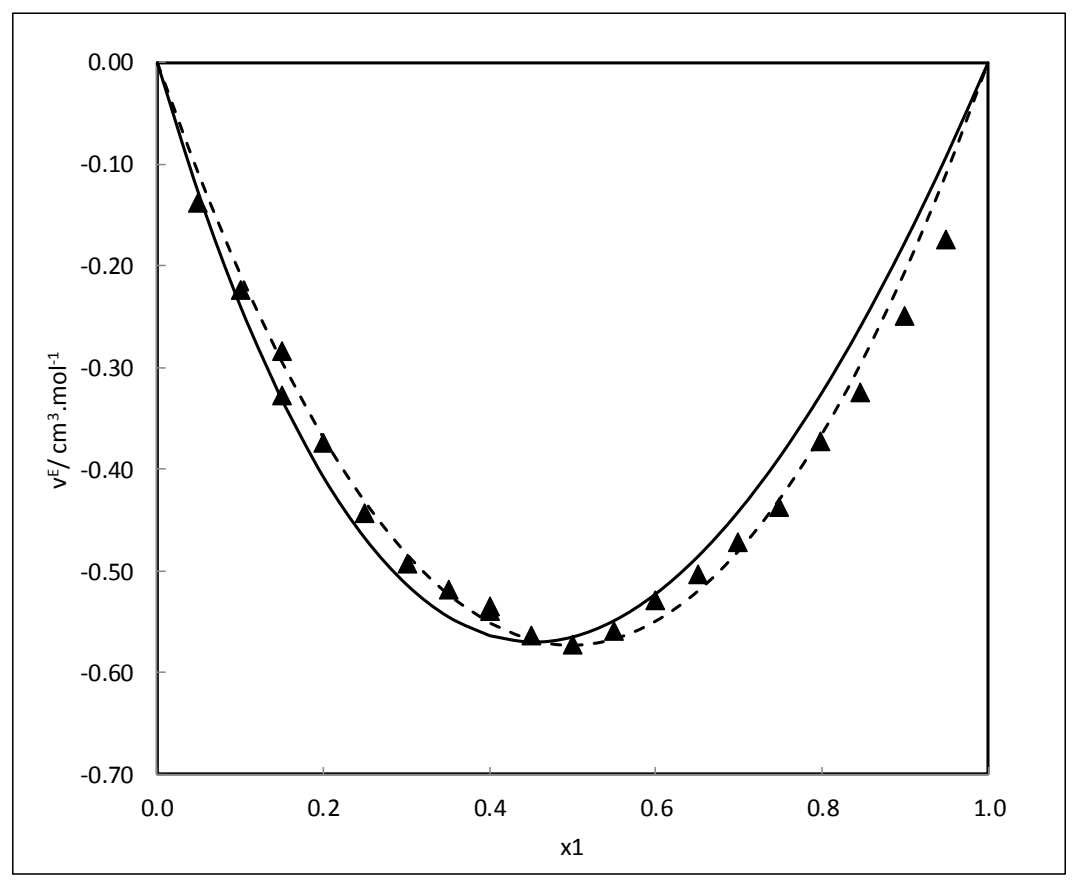

Figure 15 : Excess volumes for the Eugenol(1) + 1-Octanol(2) binary system at $293.15 \mathrm{~K}$ as a function of molar composition. Symbols: experimental data, solid line: Calculated using PFP model with constant value of $\chi_{12}$, dashed line: Calculated with PFP model with constant $\chi_{12}$ composition dependent.

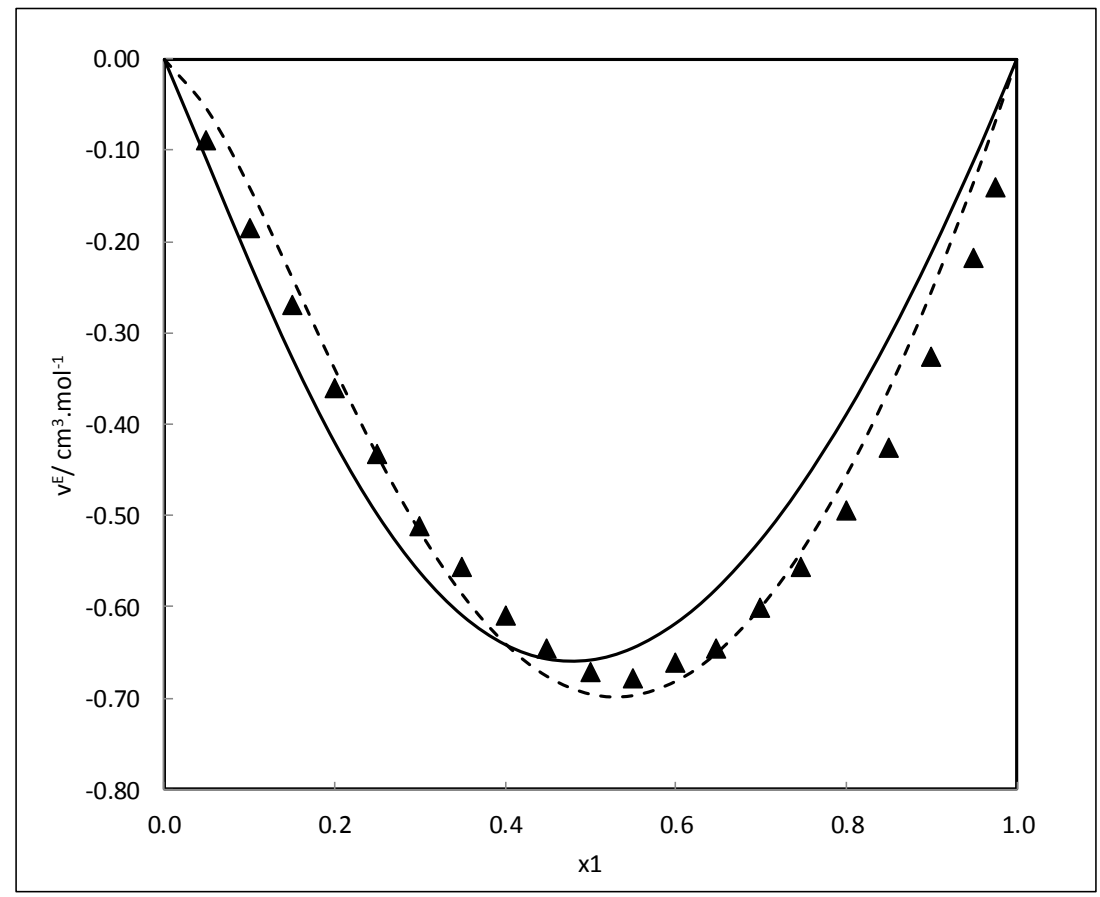

Figure 16 : Excess volumes for the Eugenol(1) $+n$-hexane(2) binary system at $293.15 \mathrm{~K}$ as a function of molar composition. Symbols: experimental data, solid line: Calculated using PFP model with constant value of $\chi_{12}$, dashed line: Calculated with PFP model with constant $\chi_{12}$ composition dependent. 


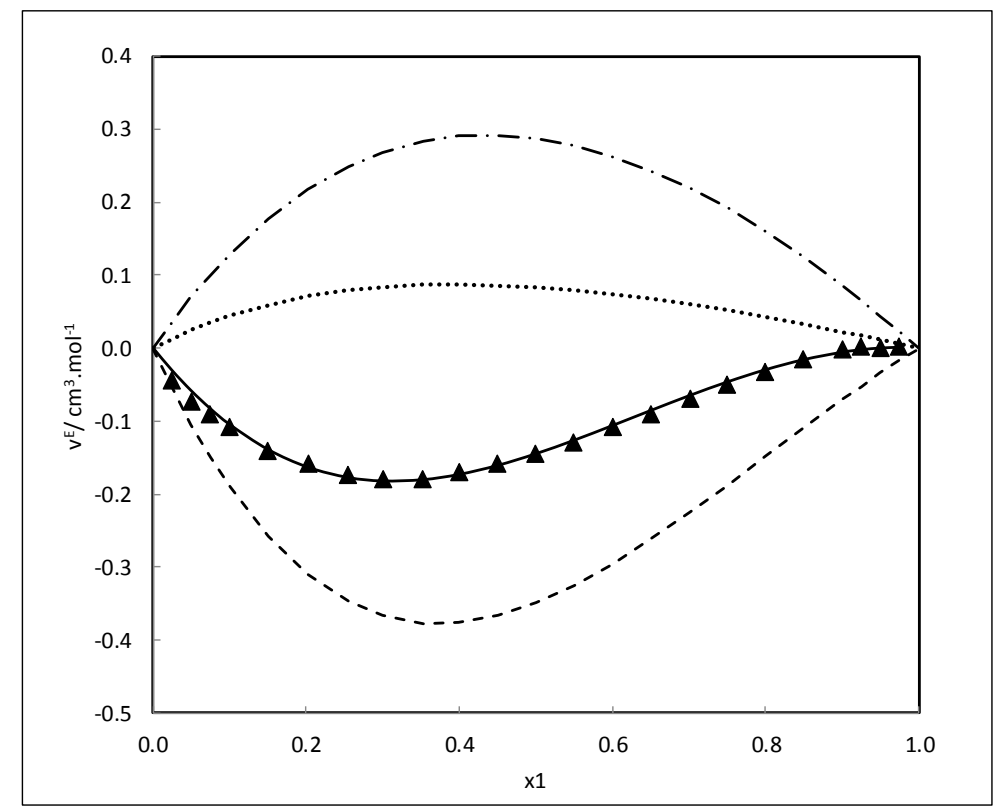

Figure 17: Excess molar volume of Furan (1) + ethanol(2) binary system at $293.15 \mathrm{~K}$ as a function of molar composition. Solid line PFP theory, Dashed line: Interactional contribution, dotted line: free volume contribution, $(-\cdot-)$ : internal pressure contribution.

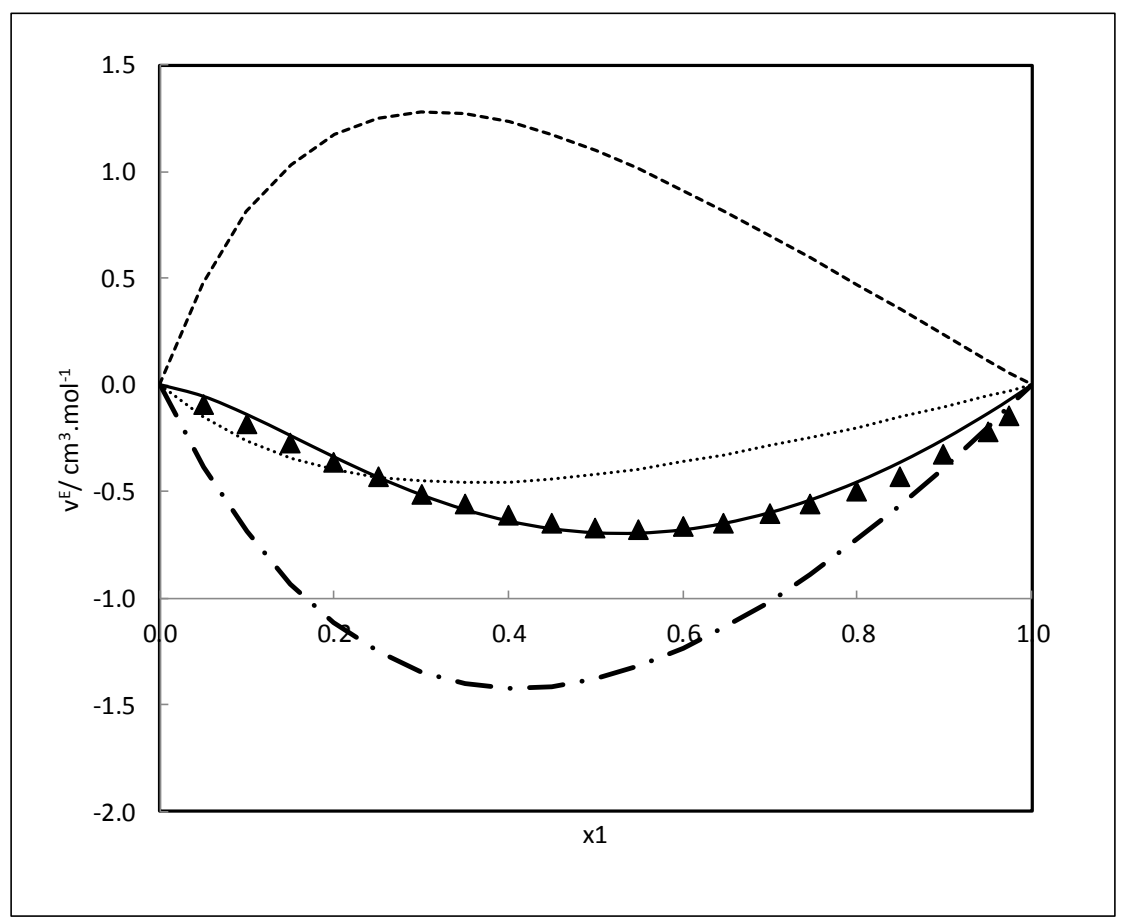

Figure 18: Excess molar volume of eugenol (1) $+\mathrm{n}$-hexane (2) binary system at $293.15 \mathrm{~K}$ as a function of molar composition. Solid line PFP theory, Dashed line: Interactional contribution, dotted line: free volume contribution, (-.-): internal pressure contribution. 
Table 21: Evaluation of the different contribution of the PFP model for the furan(1) + ethanol(2) binary system at $293.15 \mathrm{~K}$ and comparison with experimental data.

\begin{tabular}{|c|c|c|c|c|c|c|c|c|c|c|c|}
\hline \multicolumn{2}{|c|}{ Experimental data } & \multicolumn{5}{|c|}{ Constant value of $\chi_{12}$} & \multicolumn{5}{|c|}{$\chi_{12}$ composition dependent } \\
\hline \multirow{2}{*}{$\mathbf{x}_{1}$} & \multirow{2}{*}{$\mathrm{v}^{\mathrm{E}} / \mathrm{cm}^{3} \cdot \mathrm{mol}^{-1}$} & \multicolumn{3}{|c|}{ Calculated contribution $/ \mathrm{cm}^{3} \cdot \mathrm{mol}^{-1}$} & \multirow{2}{*}{$\begin{array}{c}\mathbf{v}^{\mathrm{E}} / \\
\mathrm{cm}^{3} \cdot \mathrm{mol}^{-1}\end{array}$} & \multirow{2}{*}{$\begin{array}{c}\text { Deviation / } \\
\mathrm{cm}^{3} \cdot \mathrm{mol}^{-1}\end{array}$} & \multicolumn{3}{|c|}{ Calculated contribution $/ \mathrm{cm}^{3} \cdot \mathrm{mol}^{-1}$} & \multirow{2}{*}{$\begin{array}{c}\mathbf{v}^{\mathrm{E}} / \\
\mathrm{cm}^{3} \cdot \mathrm{mol}^{-1} \\
\end{array}$} & \multirow{2}{*}{$\begin{array}{c}\text { Deviation } \\
/ \mathrm{cm}^{3} \cdot \mathrm{mol}^{-1} \\
\end{array}$} \\
\hline & & Interactional & Free volume & P* effect & & & Interactional & Free volume & P* effect & & \\
\hline 0.0248 & -0.044 & -0.035 & -0.013 & 0.036 & -0.012 & -0.032 & -0.054 & -0.013 & 0.036 & -0.031 & -0.013 \\
\hline 0.0501 & -0.074 & -0.070 & -0.025 & 0.070 & -0.024 & -0.050 & -0.105 & -0.025 & 0.070 & -0.059 & -0.015 \\
\hline 0.0748 & -0.091 & -0.101 & -0.035 & 0.101 & -0.035 & -0.056 & -0.149 & -0.035 & 0.101 & -0.083 & -0.008 \\
\hline 0.1005 & -0.108 & -0.132 & -0.044 & 0.129 & -0.047 & -0.061 & -0.191 & -0.044 & 0.129 & -0.105 & -0.003 \\
\hline 0.1505 & -0.141 & -0.187 & -0.059 & 0.178 & -0.068 & -0.073 & -0.258 & -0.059 & 0.178 & -0.139 & -0.002 \\
\hline 0.2027 & -0.159 & -0.236 & -0.071 & 0.218 & -0.089 & -0.070 & -0.311 & -0.071 & 0.218 & -0.164 & 0.005 \\
\hline 0.2549 & -0.174 & -0.276 & -0.079 & 0.248 & -0.107 & -0.067 & -0.347 & -0.079 & 0.248 & -0.178 & 0.004 \\
\hline 0.2999 & -0.180 & -0.304 & -0.084 & 0.268 & -0.120 & -0.060 & -0.366 & -0.084 & 0.268 & -0.182 & 0.002 \\
\hline 0.3526 & -0.179 & -0.329 & -0.087 & 0.283 & -0.133 & -0.046 & -0.377 & -0.087 & 0.283 & -0.181 & 0.002 \\
\hline 0.3999 & -0.169 & -0.344 & -0.087 & 0.290 & -0.142 & -0.027 & -0.376 & -0.087 & 0.290 & -0.174 & 0.005 \\
\hline 0.4500 & -0.158 & -0.353 & -0.086 & 0.292 & -0.148 & -0.010 & -0.367 & -0.086 & 0.292 & -0.161 & 0.003 \\
\hline 0.4998 & -0.145 & -0.355 & -0.084 & 0.287 & -0.151 & 0.006 & -0.349 & -0.084 & 0.287 & -0.145 & 0.000 \\
\hline 0.5499 & -0.129 & -0.349 & -0.079 & 0.278 & -0.151 & 0.022 & -0.325 & -0.079 & 0.278 & -0.127 & -0.002 \\
\hline 0.6500 & -0.090 & -0.317 & -0.067 & 0.243 & -0.141 & 0.051 & -0.262 & -0.067 & 0.243 & -0.086 & -0.004 \\
\hline 0.7009 & -0.069 & -0.290 & -0.060 & 0.219 & -0.130 & 0.061 & -0.225 & -0.060 & 0.219 & -0.066 & -0.003 \\
\hline 0.7500 & -0.050 & -0.258 & -0.052 & 0.192 & -0.117 & 0.067 & -0.187 & -0.052 & 0.192 & -0.047 & -0.003 \\
\hline 0.8000 & -0.033 & -0.218 & -0.042 & 0.160 & -0.100 & 0.067 & -0.148 & -0.042 & 0.160 & -0.030 & -0.003 \\
\hline 0.8499 & -0.016 & -0.173 & -0.033 & 0.125 & -0.080 & 0.064 & -0.109 & -0.033 & 0.125 & -0.017 & 0.001 \\
\hline 0.9000 & -0.002 & -0.121 & -0.022 & 0.086 & -0.057 & 0.055 & -0.071 & -0.022 & 0.086 & -0.006 & 0.004 \\
\hline 0.9250 & 0.002 & -0.093 & -0.017 & 0.066 & -0.044 & 0.046 & -0.052 & -0.017 & 0.066 & -0.003 & 0.005 \\
\hline 0.9500 & 0.001 & -0.063 & -0.011 & 0.045 & -0.030 & 0.031 & -0.034 & -0.011 & 0.045 & -0.001 & 0.002 \\
\hline 0.9750 & 0.003 & -0.032 & -0.006 & 0.023 & -0.015 & 0.018 & -0.017 & -0.006 & 0.023 & 0.000 & 0.003 \\
\hline
\end{tabular}


Table 22: Evaluation of the different contribution of the PFP model for the furan(1) + 1-Octanol(2) binary system at $293.15 \mathrm{~K}$ and comparison with experimental data.

\begin{tabular}{|c|c|c|c|c|c|c|c|c|c|c|c|}
\hline \multicolumn{2}{|c|}{ Experimental data } & \multicolumn{5}{|c|}{ Constant value of $\chi_{12}$} & \multicolumn{5}{|c|}{$\chi_{12}$ composition dependent } \\
\hline \multirow{2}{*}{$\mathbf{x}_{1}$} & \multirow{2}{*}{$\mathrm{v}^{\mathrm{E}} / \mathrm{cm}^{3} \cdot \mathrm{mol}^{-1}$} & \multicolumn{3}{|c|}{ Calculated contribution $/ \mathrm{cm}^{3} \cdot \mathrm{mol}^{-1}$} & \multirow{2}{*}{$\begin{array}{c}\mathbf{v}^{\mathrm{E}} / \\
\mathrm{cm}^{3} \cdot \mathrm{mol}^{-1}\end{array}$} & \multirow{2}{*}{$\begin{array}{c}\text { Deviation } \\
/ \mathrm{cm}^{3} \cdot \mathrm{mol}^{-1}\end{array}$} & \multicolumn{3}{|c|}{ Calculated contribution $/ \mathrm{cm}^{3} \cdot \mathrm{mol}^{-1}$} & \multirow{2}{*}{$\begin{array}{c}\mathbf{v}^{\mathrm{E}} / \\
\mathrm{cm}^{3} \cdot \mathrm{mol}^{-1}\end{array}$} & \multirow{2}{*}{$\begin{array}{c}\text { Deviation } \\
/ \mathrm{cm}^{3} \cdot \mathrm{mol}^{-1} \\
\end{array}$} \\
\hline & & Interactional & Free volume & P* effect & & & Interactional & Free volume & P* effect & & \\
\hline 0.0134 & 0.010 & 0.003 & -0.021 & 0.029 & 0.011 & -0.001 & 0.004 & -0.021 & 0.029 & 0.012 & -0.003 \\
\hline 0.0256 & 0.023 & 0.005 & -0.040 & 0.056 & 0.021 & 0.002 & 0.008 & -0.040 & 0.056 & 0.024 & -0.001 \\
\hline 0.0506 & 0.045 & 0.010 & -0.078 & 0.109 & 0.041 & 0.004 & 0.015 & -0.078 & 0.109 & 0.046 & -0.001 \\
\hline 0.0739 & 0.071 & 0.014 & -0.112 & 0.157 & 0.059 & 0.011 & 0.022 & -0.112 & 0.157 & 0.067 & 0.003 \\
\hline 0.0995 & 0.088 & 0.019 & -0.148 & 0.208 & 0.079 & 0.009 & 0.029 & -0.148 & 0.208 & 0.090 & -0.001 \\
\hline 0.1495 & 0.132 & 0.028 & -0.212 & 0.302 & 0.118 & 0.014 & 0.042 & -0.212 & 0.302 & 0.132 & 0.000 \\
\hline 0.1999 & 0.169 & 0.036 & -0.271 & 0.389 & 0.155 & 0.014 & 0.054 & -0.271 & 0.389 & 0.173 & -0.004 \\
\hline 0.2512 & 0.206 & 0.044 & -0.323 & 0.470 & 0.191 & 0.015 & 0.065 & -0.323 & 0.470 & 0.212 & -0.006 \\
\hline 0.3042 & 0.239 & 0.052 & -0.369 & 0.543 & 0.226 & 0.013 & 0.075 & -0.369 & 0.543 & 0.249 & -0.010 \\
\hline 0.3501 & 0.273 & 0.058 & -0.402 & 0.597 & 0.254 & 0.019 & 0.082 & -0.402 & 0.597 & 0.277 & -0.005 \\
\hline 0.4043 & 0.302 & 0.065 & -0.432 & 0.651 & 0.283 & 0.018 & 0.089 & -0.432 & 0.651 & 0.307 & -0.006 \\
\hline 0.4511 & 0.320 & 0.069 & -0.450 & 0.686 & 0.305 & 0.015 & 0.093 & -0.450 & 0.686 & 0.328 & -0.008 \\
\hline 0.5000 & 0.336 & 0.073 & -0.461 & 0.712 & 0.324 & 0.012 & 0.095 & -0.461 & 0.712 & 0.346 & -0.009 \\
\hline 0.5529 & 0.346 & 0.076 & -0.463 & 0.726 & 0.339 & 0.007 & 0.095 & -0.463 & 0.726 & 0.358 & -0.012 \\
\hline 0.6016 & 0.350 & 0.077 & -0.454 & 0.724 & 0.347 & 0.003 & 0.093 & -0.454 & 0.724 & 0.362 & -0.012 \\
\hline 0.6500 & 0.343 & 0.077 & -0.437 & 0.708 & 0.348 & -0.005 & 0.088 & -0.437 & 0.708 & 0.359 & -0.016 \\
\hline 0.7000 & 0.326 & 0.075 & -0.409 & 0.674 & 0.340 & -0.015 & 0.081 & -0.409 & 0.674 & 0.347 & -0.021 \\
\hline 0.7499 & 0.305 & 0.070 & -0.370 & 0.622 & 0.323 & -0.018 & 0.072 & -0.370 & 0.622 & 0.324 & -0.019 \\
\hline 0.8010 & 0.271 & 0.063 & -0.318 & 0.547 & 0.292 & -0.021 & 0.060 & -0.318 & 0.547 & 0.288 & -0.017 \\
\hline 0.8500 & 0.222 & 0.053 & -0.257 & 0.453 & 0.249 & -0.026 & 0.046 & -0.257 & 0.453 & 0.241 & -0.019 \\
\hline 0.9010 & 0.164 & 0.040 & -0.182 & 0.329 & 0.186 & -0.023 & 0.030 & -0.182 & 0.329 & 0.177 & -0.013 \\
\hline 0.9250 & 0.126 & 0.032 & -0.142 & 0.260 & 0.149 & -0.024 & 0.023 & -0.142 & 0.260 & 0.141 & -0.015 \\
\hline 0.9500 & 0.089 & 0.022 & -0.098 & 0.182 & 0.106 & -0.017 & 0.015 & -0.098 & 0.182 & 0.098 & -0.010 \\
\hline 0.9750 & 0.044 & 0.012 & -0.050 & 0.095 & 0.056 & -0.012 & 0.007 & -0.050 & 0.095 & 0.052 & -0.008 \\
\hline
\end{tabular}


Table 23: Evaluation of the different contribution of the PFP model for the Eugenol(1) + 1-Octanol(2) binary system at $293.15 \mathrm{~K}$ and comparison with experimental data.

\begin{tabular}{|c|c|c|c|c|c|c|c|c|c|c|c|}
\hline \multicolumn{2}{|c|}{ Experimental data } & \multicolumn{5}{|c|}{ Constant value of $\chi_{12}$} & \multicolumn{5}{|c|}{$\chi_{12}$ composition dependent } \\
\hline \multirow{2}{*}{$\mathbf{x}_{1}$} & \multirow{2}{*}{$\mathrm{v}^{\mathrm{E}} / \mathrm{cm}^{3} \cdot \mathrm{mol}^{-1}$} & \multicolumn{3}{|c|}{ Calculated contribution $/ \mathrm{cm}^{3} \cdot \mathrm{mol}^{-1}$} & \multirow{2}{*}{$\begin{array}{c}\mathbf{v}^{\mathrm{E}} / \\
\mathrm{cm}^{3} \cdot \mathrm{mol}^{-1}\end{array}$} & \multirow{2}{*}{$\begin{array}{l}\text { Deviation } \\
/ \mathrm{cm}^{3} \cdot \mathrm{mol}^{-1}\end{array}$} & \multicolumn{3}{|c|}{ Calculated contribution $/ \mathrm{cm}^{3} \cdot \mathrm{mol}^{-1}$} & \multirow{2}{*}{$\begin{array}{c}\mathbf{v}^{\mathrm{E}} / \\
\mathrm{cm}^{3} \cdot \mathrm{mol}^{-1}\end{array}$} & \multirow{2}{*}{$\begin{array}{l}\text { Deviation } \\
/ \mathrm{cm}^{3} \cdot \mathrm{mol}^{-1}\end{array}$} \\
\hline & & Interactional & Free volume & P* effect & & & Interactional & Free volume & P* effect & & \\
\hline 0.0500 & -0.137 & -0.12278 & -0.0001 & -0.006 & -0.129 & -0.008 & -0.104 & -0.0001 & -0.006 & -0.110 & -0.003 \\
\hline 0.1001 & -0.223 & -0.22760 & -0.0001 & -0.012 & -0.240 & 0.016 & -0.196 & -0.0001 & -0.012 & -0.208 & -0.001 \\
\hline 0.1500 & -0.328 & -0.31515 & -0.0002 & -0.017 & -0.332 & 0.004 & -0.278 & -0.0002 & -0.017 & -0.295 & -0.001 \\
\hline 0.1502 & -0.285 & -0.31547 & -0.0002 & -0.017 & -0.332 & 0.048 & -0.278 & -0.0002 & -0.017 & -0.295 & 0.003 \\
\hline 0.2002 & -0.374 & -0.38736 & -0.0002 & -0.020 & -0.408 & 0.034 & -0.349 & -0.0002 & -0.020 & -0.369 & -0.001 \\
\hline 0.2502 & -0.443 & -0.44432 & -0.0002 & -0.023 & -0.468 & 0.025 & -0.409 & -0.0002 & -0.023 & -0.432 & 0.000 \\
\hline 0.3004 & -0.492 & -0.48761 & -0.0002 & -0.026 & -0.514 & 0.021 & -0.458 & -0.0002 & -0.026 & -0.484 & -0.004 \\
\hline 0.3502 & -0.518 & -0.51742 & -0.0003 & -0.027 & -0.545 & 0.027 & -0.496 & -0.0003 & -0.027 & -0.523 & -0.006 \\
\hline 0.4001 & -0.539 & -0.53502 & -0.0003 & -0.028 & -0.564 & 0.025 & -0.523 & -0.0003 & -0.028 & -0.551 & -0.010 \\
\hline 0.4003 & -0.536 & -0.53508 & -0.0003 & -0.028 & -0.564 & 0.028 & -0.523 & -0.0003 & -0.028 & -0.552 & -0.005 \\
\hline 0.4501 & -0.563 & -0.54107 & -0.0003 & -0.029 & -0.570 & 0.007 & -0.539 & -0.0003 & -0.029 & -0.568 & -0.006 \\
\hline 0.5002 & -0.572 & -0.53614 & -0.0003 & -0.028 & -0.565 & -0.007 & -0.545 & -0.0003 & -0.028 & -0.573 & -0.008 \\
\hline 0.5502 & -0.559 & -0.52086 & -0.0002 & -0.028 & -0.549 & -0.010 & -0.539 & -0.0002 & -0.028 & -0.567 & -0.009 \\
\hline 0.6000 & -0.529 & -0.49588 & -0.0002 & -0.026 & -0.522 & -0.006 & -0.523 & -0.0002 & -0.026 & -0.550 & -0.012 \\
\hline 0.6502 & -0.503 & -0.46144 & -0.0002 & -0.024 & -0.486 & -0.017 & -0.496 & -0.0002 & -0.024 & -0.520 & -0.012 \\
\hline 0.6992 & -0.472 & -0.41932 & -0.0002 & -0.022 & -0.442 & -0.030 & -0.458 & -0.0002 & -0.022 & -0.481 & -0.016 \\
\hline 0.7482 & -0.438 & -0.36921 & -0.0002 & -0.020 & -0.389 & -0.049 & -0.411 & -0.0002 & -0.020 & -0.431 & -0.021 \\
\hline 0.7984 & -0.373 & -0.31005 & -0.0001 & -0.017 & -0.327 & -0.046 & -0.351 & -0.0001 & -0.017 & -0.368 & -0.019 \\
\hline 0.8457 & -0.325 & -0.24735 & -0.0001 & -0.013 & -0.261 & -0.064 & -0.285 & -0.0001 & -0.013 & -0.298 & -0.017 \\
\hline 0.8997 & -0.249 & -0.16804 & -0.0001 & -0.009 & -0.177 & -0.072 & -0.197 & -0.0001 & -0.009 & -0.206 & -0.019 \\
\hline 0.9499 & -0.174 & -0.08712 & -0.00004 & -0.005 & -0.092 & -0.082 & -0.104 & -0.00004 & -0.005 & -0.109 & -0.013 \\
\hline
\end{tabular}


Table 24: Evaluation of the different contribution of the PFP model for the Eugenol(1) + $n$-hexane(2) binary system at $293.15 \mathrm{~K}$ and comparison with experimental data.

\begin{tabular}{|c|c|c|c|c|c|c|c|c|c|c|c|}
\hline \multicolumn{2}{|c|}{ Experimental data } & \multicolumn{5}{|c|}{ Constant value of $\chi_{12}$} & \multicolumn{5}{|c|}{$\chi_{12}$ composition dependent } \\
\hline \multirow{2}{*}{$\mathbf{x}_{1}$} & \multirow{2}{*}{$\mathbf{v}^{\mathrm{E}} / \mathrm{cm}^{3} \cdot \mathrm{mol}^{-1}$} & \multicolumn{3}{|c|}{ Calculated contribution $/ \mathrm{cm}^{3} \cdot \mathrm{mol}^{-1}$} & \multirow{2}{*}{$\begin{array}{c}\mathbf{v}^{\mathrm{E}} / \\
\mathrm{cm}^{3} \cdot \mathrm{mol}^{-1} \\
\end{array}$} & \multirow{2}{*}{$\begin{array}{l}\text { Deviation } \\
/ \mathrm{cm}^{3} \cdot \mathrm{mol}^{-1}\end{array}$} & \multicolumn{3}{|c|}{ Calculated contribution $/ \mathrm{cm}^{3} \cdot \mathrm{mol}^{-1}$} & \multirow{2}{*}{$\begin{array}{c}\mathbf{v}^{\mathrm{E}} / \\
\mathrm{cm}^{3} \cdot \mathrm{mol}^{-1}\end{array}$} & \multirow{2}{*}{$\begin{array}{c}\text { Deviation } \\
/ \mathrm{cm}^{3} \cdot \mathrm{mol}^{-1}\end{array}$} \\
\hline & & Interactional & Free volume & P* effect & & & Interactional & Free volume & P* effect & & \\
\hline 0.0500 & -0.089 & 0.421 & -0.150 & -0.383 & -0.111 & 0.022 & 0.478 & -0.150 & -0.383 & -0.055 & -0.035 \\
\hline 0.1000 & -0.185 & 0.727 & -0.261 & -0.689 & -0.223 & 0.038 & 0.810 & -0.261 & -0.689 & -0.140 & -0.045 \\
\hline 0.1500 & -0.270 & 0.945 & -0.341 & -0.931 & -0.328 & 0.057 & 1.033 & -0.341 & -0.931 & -0.239 & -0.031 \\
\hline 0.2000 & -0.360 & 1.093 & -0.397 & -1.116 & -0.421 & 0.061 & 1.174 & -0.397 & -1.116 & -0.340 & -0.020 \\
\hline 0.2500 & -0.433 & 1.185 & -0.433 & -1.252 & -0.499 & 0.066 & 1.251 & -0.433 & -1.252 & -0.434 & 0.001 \\
\hline 0.3000 & -0.512 & 1.235 & -0.453 & -1.345 & -0.563 & 0.051 & 1.280 & -0.453 & -1.345 & -0.517 & 0.005 \\
\hline 0.3500 & -0.557 & 1.248 & -0.459 & -1.399 & -0.610 & 0.053 & 1.272 & -0.459 & -1.399 & -0.587 & 0.030 \\
\hline 0.4000 & -0.609 & 1.233 & -0.455 & -1.420 & -0.642 & 0.032 & 1.235 & -0.455 & -1.420 & -0.640 & 0.031 \\
\hline 0.4491 & -0.646 & 1.196 & -0.442 & -1.412 & -0.658 & 0.012 & 1.178 & -0.442 & -1.412 & -0.676 & 0.030 \\
\hline 0.5000 & -0.672 & 1.138 & -0.421 & -1.375 & -0.659 & -0.013 & 1.101 & -0.421 & -1.375 & -0.696 & 0.024 \\
\hline 0.5489 & -0.678 & 1.066 & -0.395 & -1.317 & -0.646 & -0.032 & 1.015 & -0.395 & -1.317 & -0.697 & 0.020 \\
\hline 0.6000 & -0.660 & 0.977 & -0.362 & -1.234 & -0.618 & -0.042 & 0.915 & -0.362 & -1.234 & -0.681 & 0.021 \\
\hline 0.6473 & -0.646 & 0.885 & -0.328 & -1.138 & -0.581 & -0.065 & 0.815 & -0.328 & -1.138 & -0.651 & 0.005 \\
\hline 0.6994 & -0.600 & 0.774 & -0.287 & -1.014 & -0.527 & -0.073 & 0.701 & -0.287 & -1.014 & -0.601 & 0.000 \\
\hline 0.7464 & -0.556 & 0.666 & -0.247 & -0.887 & -0.468 & -0.087 & 0.594 & -0.247 & -0.887 & -0.541 & -0.015 \\
\hline 0.8000 & -0.494 & 0.536 & -0.199 & -0.727 & -0.390 & -0.105 & 0.470 & -0.199 & -0.727 & -0.456 & -0.038 \\
\hline 0.8499 & -0.427 & 0.409 & -0.151 & -0.563 & -0.306 & -0.121 & 0.352 & -0.151 & -0.563 & -0.362 & -0.064 \\
\hline 0.8991 & -0.327 & 0.279 & -0.103 & -0.390 & -0.214 & -0.113 & 0.237 & -0.103 & -0.390 & -0.256 & -0.071 \\
\hline 0.950 & -0.218 & 0.141 & -0.052 & -0.199 & -0.111 & -0.107 & 0.117 & -0.052 & -0.199 & -0.134 & -0.084 \\
\hline 0.9751 & -0.141 & 0.070 & -0.026 & -0.100 & -0.056 & -0.085 & 0.058 & -0.026 & -0.100 & -0.068 & -0.073 \\
\hline
\end{tabular}




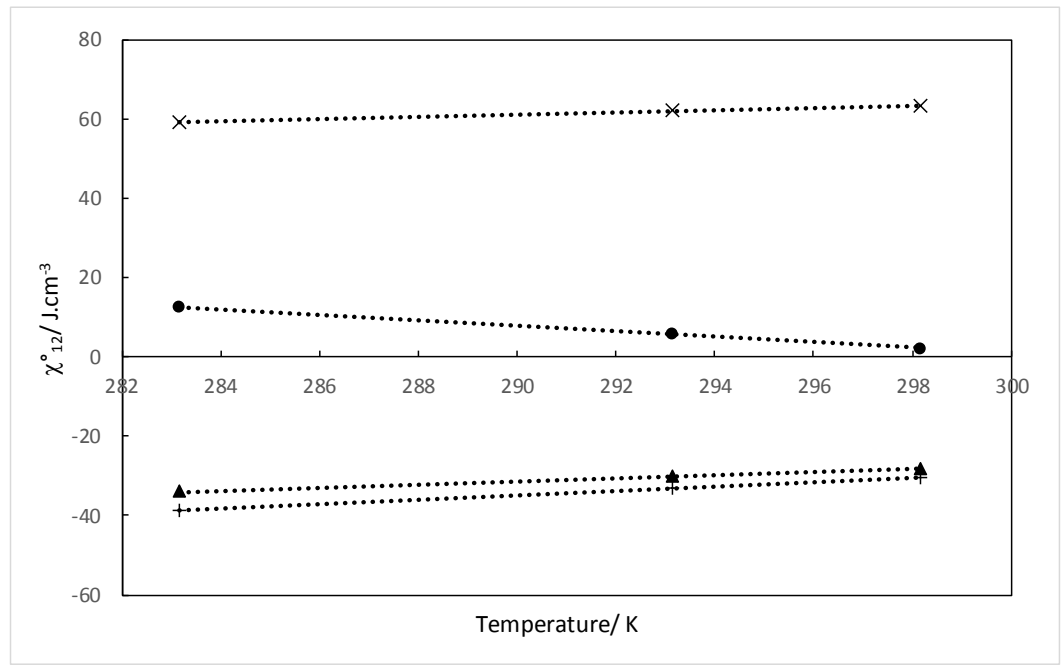

Figure 19: Evolution of $\chi_{12}^{\circ}$ parameters as a function of temperature. $(\Delta)$ : furan + ethanol binary system, (+): eugenol + 1-Octanol binary system, (x): eugenol + $n$-hexane binary system, $(\bullet)$ : furan + 1-Octanol binary system.

\section{Conclusion}

The densities of 4 binary mixtures (Eugenol $+n$-hexane, Eugenol +1 -Octanol, Furan + Ethanol and Furan +1 -Octanol) were measured over the temperature range $\mathrm{T}=(278.15-323.15 \mathrm{~K})$. Densities and speeds of sound were also measured for each pure component. Deduced from these properties, the excess molar volumes $\mathrm{V}^{\mathrm{E}}$ of these systems are well correlated by the Redlich-Kister correlation. Except for the furan +1 -Octanol binary systems, all the excess volumes are negative. Concerning the furan + ethanol binary system in the dilute region of ethanol, excess molar volume becomes positive when the temperature increases. The capability of the Prigogine-Flory-Patterson (PFP) model to predict the excess molar volume is tested on these binary mixtures. The PFP theory faces more difficulties in the restitution of the experimental $V^{E}$ for furan + ethanol binary system. For this system, it appears that the most relevant contribution is attributes to interactional interaction. $A$ tentative of utilization of a $\chi_{12}$ composition dependent was done and the results are better represented.

\section{Acknowledgments}

Financial support from the ANR of France through the project Memobiol (ANR-09-CP2D-10-04 MEMOBIOL) is gratefully acknowledged. 


\section{References}

1. Huber, G. W. , Iborra, S.,Corma, A.: Synthesis of transportation fuels from biomass: chemistry, catalysts, and engineering. Chem. Rev., 106,4044-98 (2009)

2. Auger, E., Coquelet, C., Valtz, A., Nala, M., Naidoo, P., Ramjugernath, D.: Equilibrium data and GCPC SAFT predictions for furanic extraction. Fluid Phase Equilib., 430, 57-66 (2016)

3. Gladstone, J.H.:Refraction-equivalents of organic compounds. J. Chem. Soc., 45, 241-259 (1884)

4. Redlich, O.Kister, A.: Algebraic Representation of Thermodynamic Properties and the Classification of Solutions. Ind. Eng. Chem., 40, 345-348 (1948)

5. Desnoyers, J., Perron, G.: Treatment of Excess Thermodynamic Quantities for Liquid Mixtures. J. Solution Chem., 26, 749-755 (1997)

6. Rowley, R. L.: DIPPR ${ }^{\circledR}$ Data Compilation of Pure Chemical Properties. Design Institute for Physical Properties (2010)

7. Sharma, N., Kumar, D.: Study and Design of Eugenol Derivatives as Potent Antioxidant Using Quantum Mechanical Method. Int. J. Applied Pharmaceutical and Biological Research, 1, 24-32 (2016)

8. Patterson, D., Delmas, G.: Corresponding states theories and liquid models. Discuss. Faraday Soc., 49, 98-105 (1970)

9. Gepert, M., Zorębski, E., Leszczyńska, A.: Is Flory's model the best tool for studying the thermodynamic properties of any kind of binary mixtures? Fluid Phase Equilib., 233, 157-169 (2005)

10. Galvao, A.C., Francesconi, A.Z.: Application of the Prigogine -Flory-Patterson model to excess molar enthalpy of binary liquid mixtures containing acetonitrile and 1-alkanol, J. molecular Liquids, $107,127-139$ (2003)

11. Torres, R.B., Pina, C.G., Francesconi, A.Z.: Application of the Prigogine -Flory-Patterson theory to excess molar volume of binary mixtures of acetonitrile with 1-alkanols. J. molecular Liquids, 139, 110116 (2008)

12. Piñeiro, Á., Amigo, A., Bravo, R., Brocos, P.: Re-examination and symmetrization of the adjustable parameters of the ERAS model.Fluid Phase Equilib., 173, 211-239 (2000)

13. Flory, P.J.: Statistical Thermodynamics of Liquid Mixtures. J. Am. Chem. Soc., 87, 1833-1838 (1965)

14. Valtz, A., Coquelet, C., Boukais-Belaribi, G., Dahmani, A., Belaribi, F.B.: Volumetric Properties of Binary Mixtures of 1,2-Dichloroethane with Polyethers from (283.15 to 333.15) K and at Atmospheric Pressure. J. Chem. Eng. Data, 56, 1629-1657 (2011) 
15. Iloukhani, H.,Rezaei-Sameti, M.:Volumetric properties of methylcyclohexane with n-alkanes (C5C10) at 293.15, 298.15 and $303.15 \mathrm{~K}$-comparison with Prigogine-Flory-Patterson theory. J. Mol. Liq., 126, 62-68 (2006)

16. Lemmon, E.W., Huber, M.L., McLinden, M.O.: NIST Standard Reference Database 23: Reference Fluid Thermodynamic and Transport Properties-REFPROP, Version 10, National Institute of Standards and Technology (2013)

17. Guthrie Jr, G. B., Scott, D. W., Hubbard, W. N., Katz, C., McCullough, J. P., Gross, M. E., Williamson, K.D., Waddington, G.:Thermodynamic properties of furan, Journal of the American Chemical Society, 74, 4662-4669 (1952).

18. Karabaev, M.K.: Kinetische, thermische und kalorische Eigenschaften von fluessigen Eugenol. Izv. Akad. Nauk Uzb. SSR Ser. Fiz. Mat. Nauk, 72-74 (1983)

19. Mel'nikov, G. A.; Vervenko, V. N.; Otpuschennikov, N. F.: Complex Study of the Elastic and Thermal Properties of Hydrocarbons and Their Halogen Derivatives by the Acoustic Method. Zh. Fiz. Khim., 62, 798 (1998)

20. Rubini, K., Francesconi, R., Bigi, A., Comelli, F.:Excess molar enthalpies and heat capacities of dimethyl sulfoxide + seven normal alkanols at $303.15 \mathrm{~K}$ and atmospheric pressure. Thermochim. Acta, 452, 124-127 (2007)

21. Hansen, C. M.: Hansen solubility parameters: a user's handbook. CRC press (2002)

22. Mulder, M.H.V., Smolders, C.A.: On the mechanisms of separation of ethanol/water mixtures by pervaporation. I. Calculations of concentration profiles. J. Membrane Science, 17, 289-307 (1984) 


\title{
Density and excess volume for four systems involving eugenol and furan
}

\author{
Eric Auger, Christophe Coquelet, Alain Valtz
}

Mines ParisTech PSL University, CTP-Centre of Thermodynamics of Processes, 35 Rue Saint Honoré, 77305 Fontainebleau, France.

\section{Supplementary information}

\section{Comparison with literature data}

We have compared our measured density data with literature data for 1-octanol, furan and eugenol. We have also considered the DIPPR [1] correlation (equation $n^{\circ} 105$ ) (Eq. S1) available in Simulis ${ }^{\mathrm{TM}}$ Thermodynamics software from PROSIM France. For these three components, we have plotted a parity graph, experimental value vs calculated values.

$$
\rho=\frac{A}{B^{\left(1+\left(1-\frac{T}{C}\right)^{E}\right)}}
$$

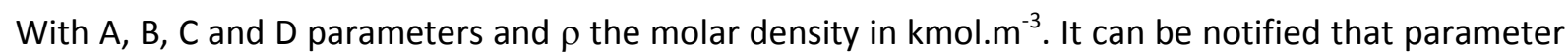
$C$ corresponds to the critical temperature. Concerning eugenol, we have used boiling temperature. Table S1 shows the different parameters. We have adjusted the parameters using literature data for the furan and eugenol and applied the Eq. S1 with available parameters for 1-octanol.

Table S1: DIPPR correlation parameters for 1-Octanol, furan and Eugenol.

\begin{tabular}{cccccc}
\hline Component & $\mathrm{A}$ & $\mathrm{B}$ & $\mathrm{C}$ & $\mathrm{D}$ & $\mathrm{AAD}: \%$ \\
\hline 1-Octanol $^{*}$ & 0.0048979 & 0.24931 & 652.3 & 0.27824 & 0.44 \\
Furan & 0.0015357 & 0.287097 & 490.2 & 0.303716 & 0.03 \\
Eugenol & 0.0032754 & 0.638032 & 523 & 0.829143 & 0.19 \\
\hline
\end{tabular}

AAD: Average Absolute Deviation on literature data, ${ }^{*}$ from Simulis ${ }^{\mathrm{TM}}$ Thermodynamics

Concerning 1-Octanol, we have used the data from TDE (NIST) [2] available in ASPEN plus software [3]. Concerning furan we have used the data from Gunthrie et al. [4] and Timmermans and HennautRoland [5]. Concerning eugenol we have used the data from Bingham and Spooner [6]. 


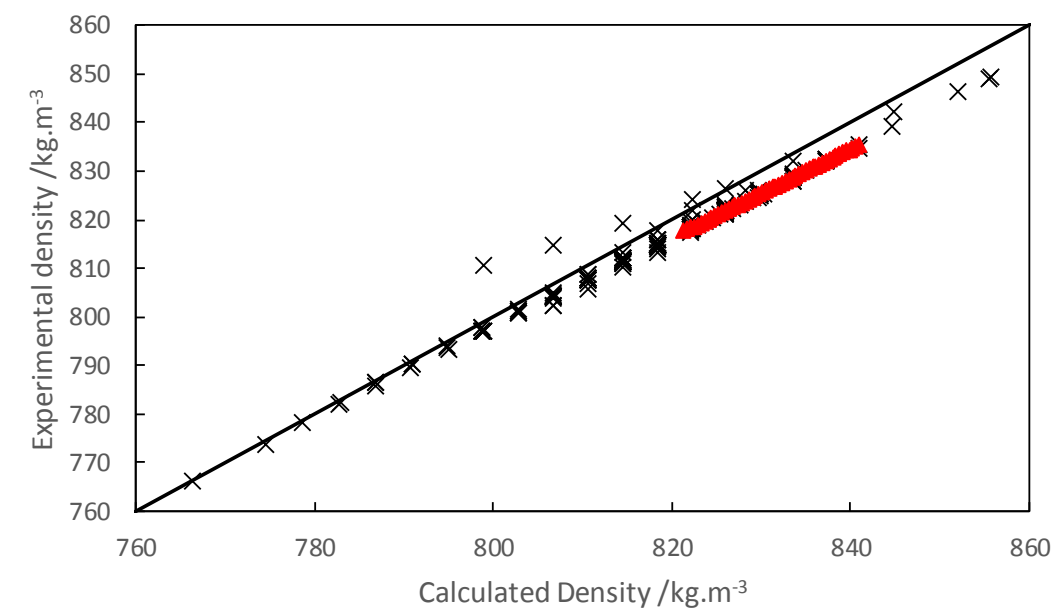

Figure S1 : Parity graph concerning 1-Octanol. (x): literature data, red symbol: This work (Table 2).

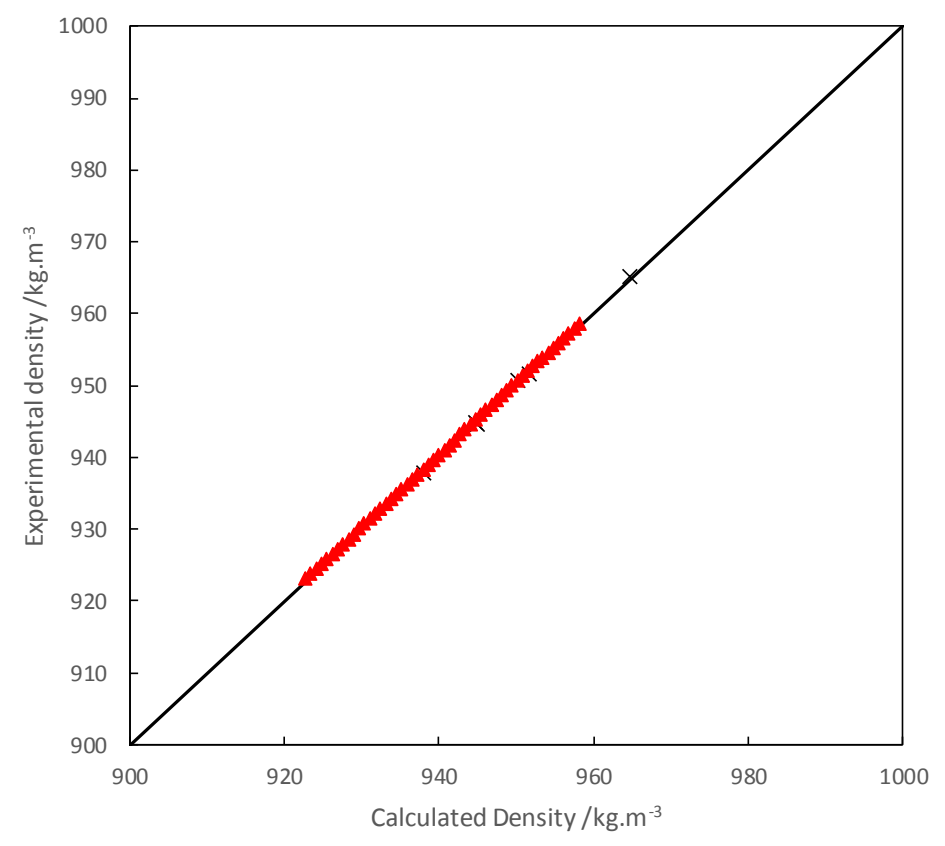

Figure S2 : Parity graph concerning furan. $(x)$ : literature data, red symbol: This work (Table 2). 


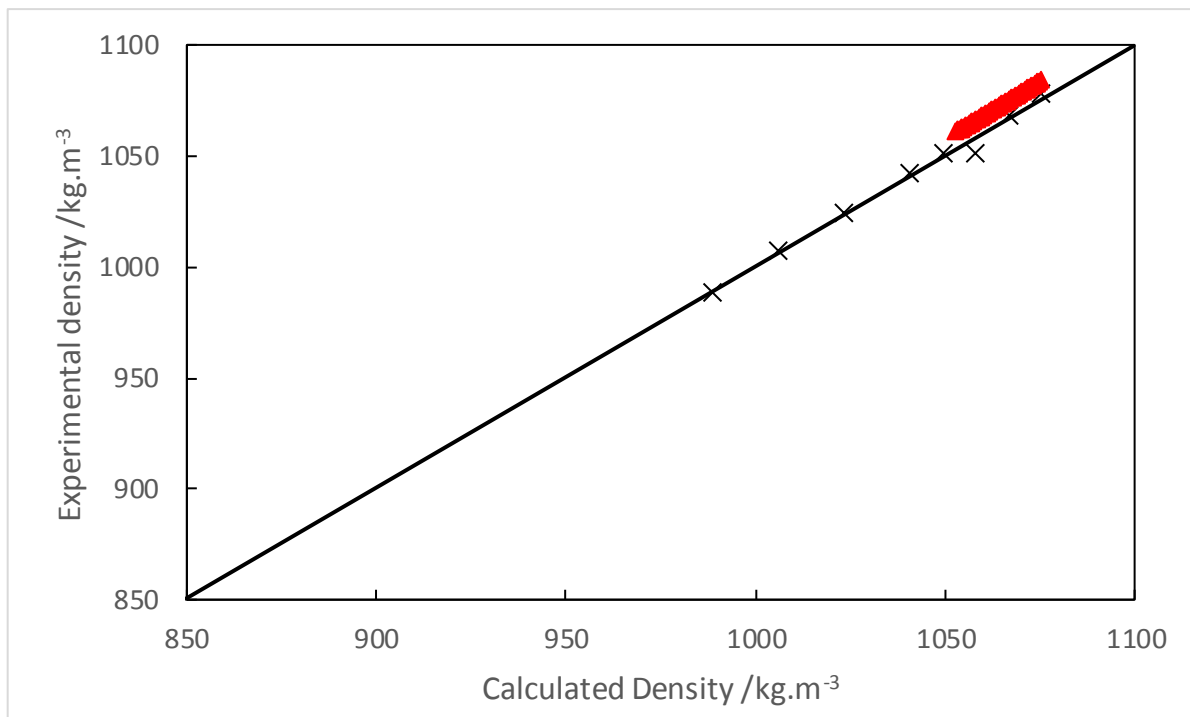

Figure S3 : Parity graph concerning eugenol. (x): literature data, red symbol: This work (Table 2).

Concerning ethanol and $\mathrm{n}$-hexane we have directly compared our values with the predicted value from REFPROP 10.0. Figs. S4 and S5 show the parity graph.

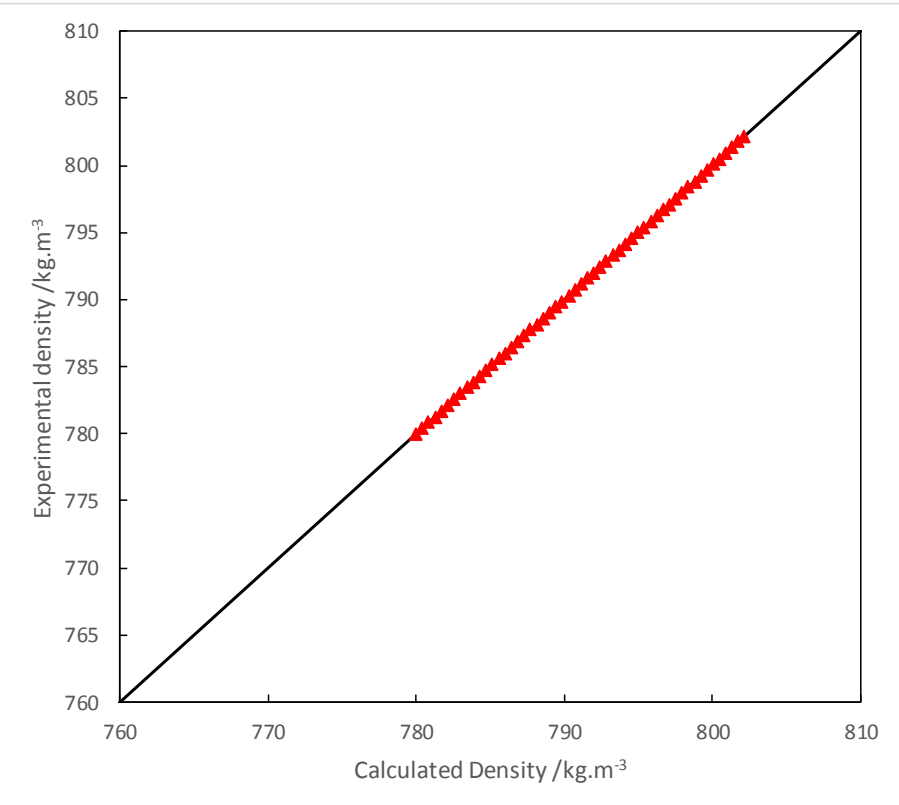

Figure S4 : Parity graph concerning ethanol. red symbol: This work (Table 2). 


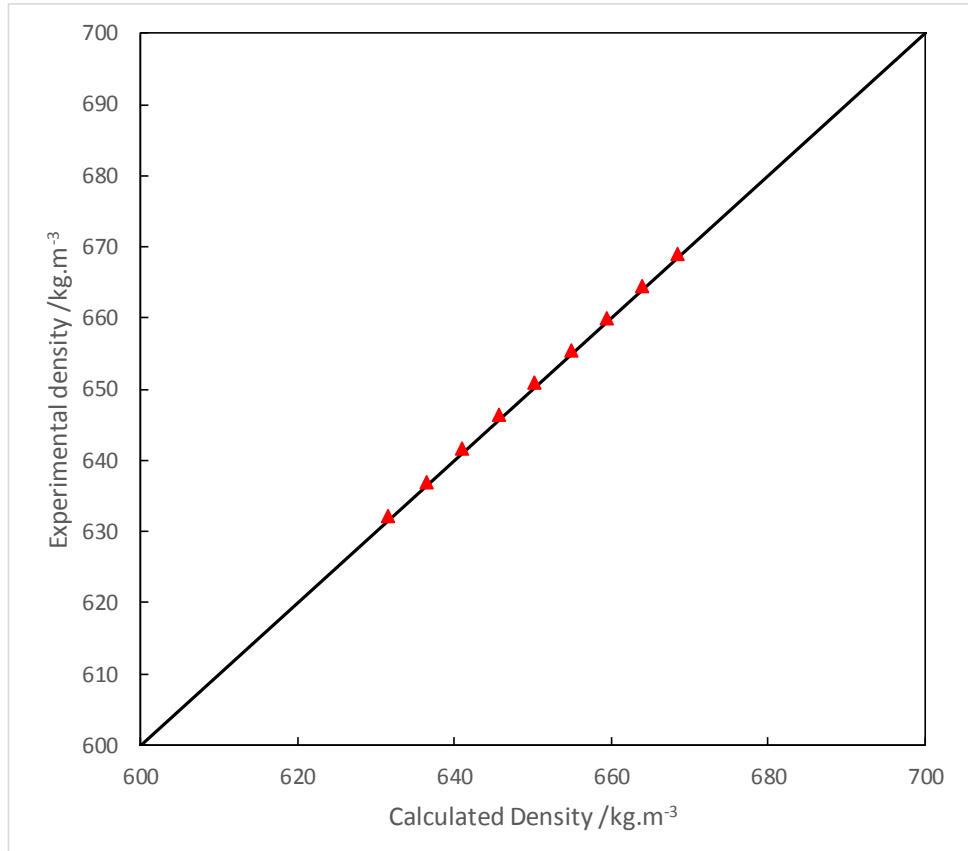

Figure S5 : Parity graph concerning n-hexane. red symbol: This work (Table 2).

According to Figs. S1 to S5, we can conclude that our pure component density data are in good agreement with literature data and consistent.

2. Prigogine Flory Patterson (PFP) model

In this part of the $\mathrm{SI}$ additional results concerning the modeling of the excess volume by using Prigogine Flory Patterson (PFP) model at 298 and 283K are presented (Figs. S6 to S13, Tables S2 to S9). 


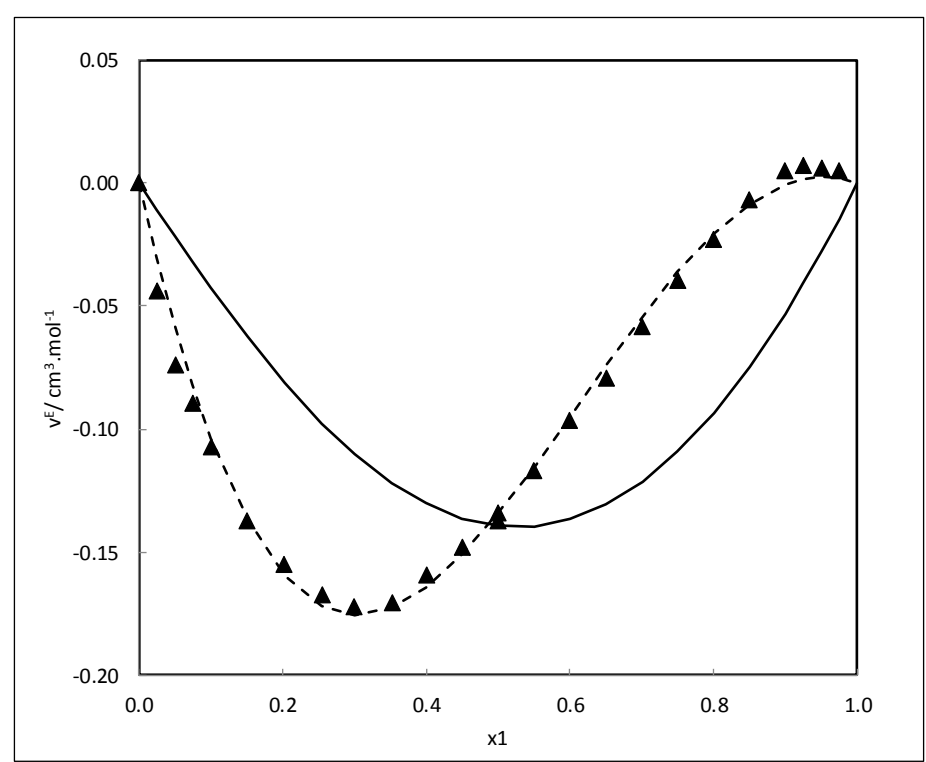

Figure S6 : Excess volumes for the furan(1) + Ethanol(2) binary system at $298 \mathrm{~K}$ as a function of molar composition. Symbols: experimental data, solid line: Calculated using PFP model with constant value of $\chi_{12}$, dashed line: Calculated with PFP model with constant $\chi_{12}$ composition dependent.

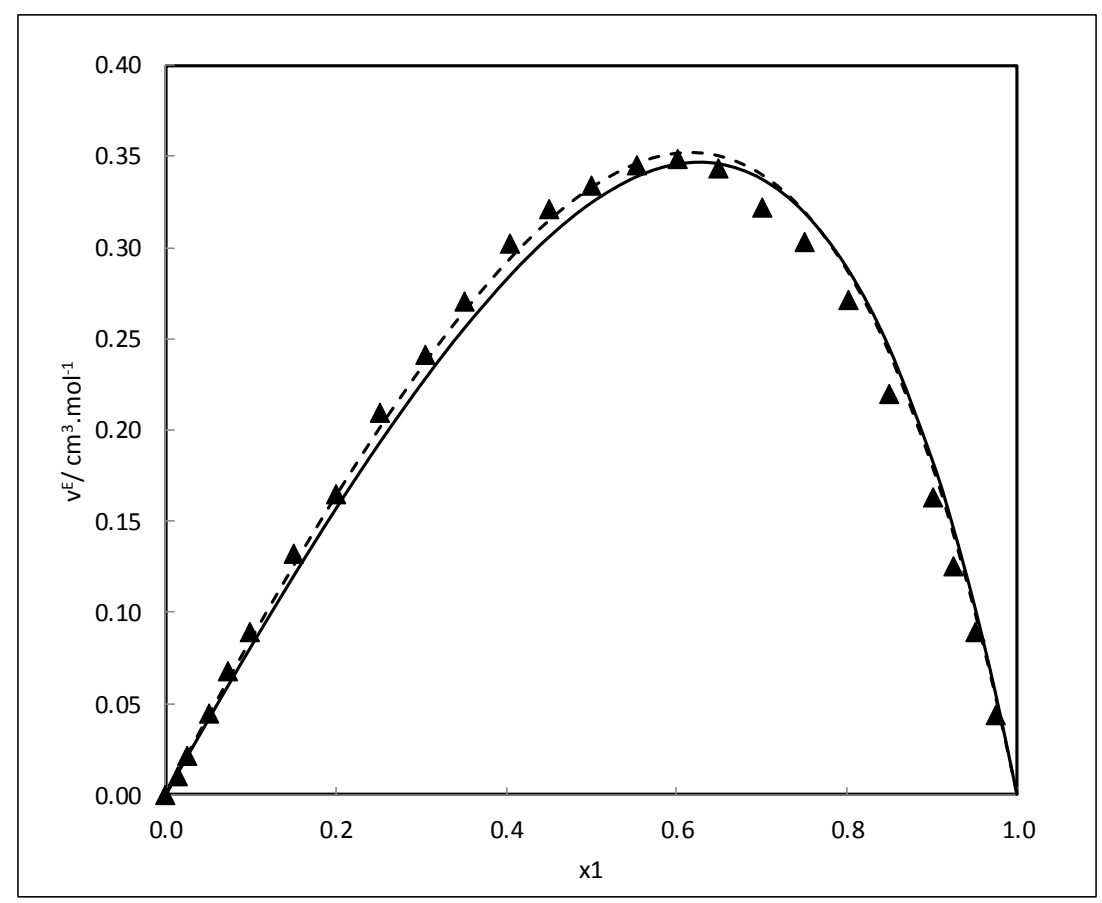

Figure S7: Excess volumes for the Furan(1) + 1-Octanol(2) binary system at $298 \mathrm{~K}$ as a function of molar composition. Symbols: experimental data, solid line: Calculated using PFP model with constant value of $\chi_{12}$, dashed line: Calculated with PFP model with constant $\chi_{12}$ composition dependent. 


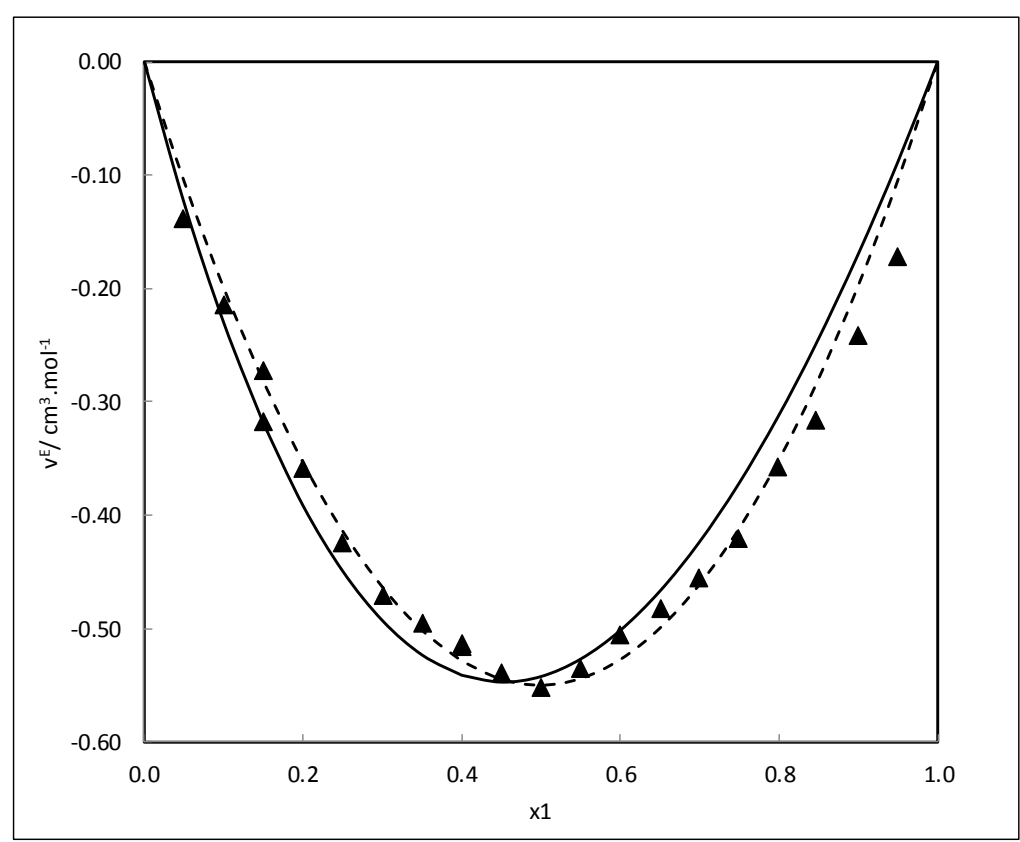

Figure S8 : Excess volumes for the Eugenol(1) + 1-Octanol(2) binary system at 298K as a function of molar composition. Symbols: experimental data, solid line: Calculated using PFP model with constant value of $\chi_{12}$, dashed line: Calculated with PFP model with constant $\chi_{12}$ composition dependent.

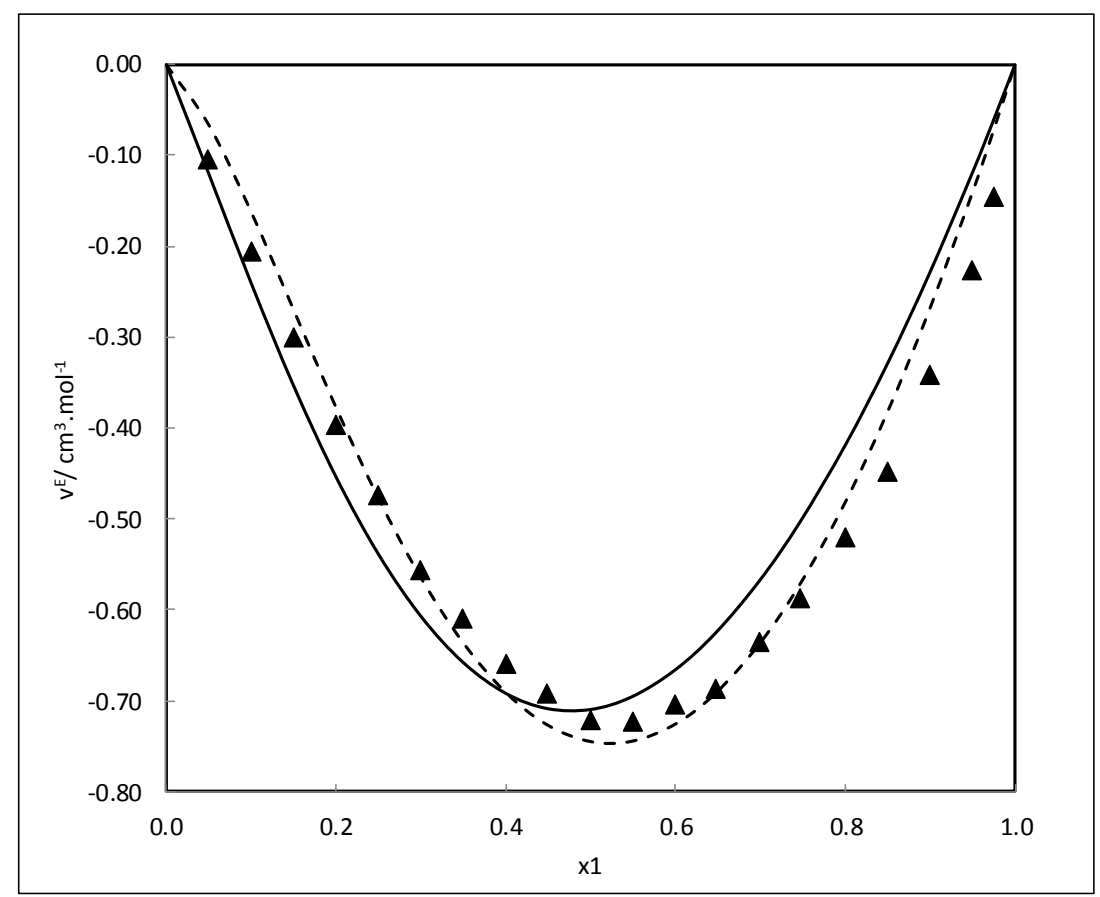

Figure S9 : Excess volumes for the Eugenol(1) $+n$-hexane(2) binary system at $298 \mathrm{~K}$ as a function of molar composition. Symbols: experimental data, solid line: Calculated using PFP model with constant value of $\chi_{12}$, dashed line: Calculated with PFP model with constant $\chi_{12}$ composition dependent. 


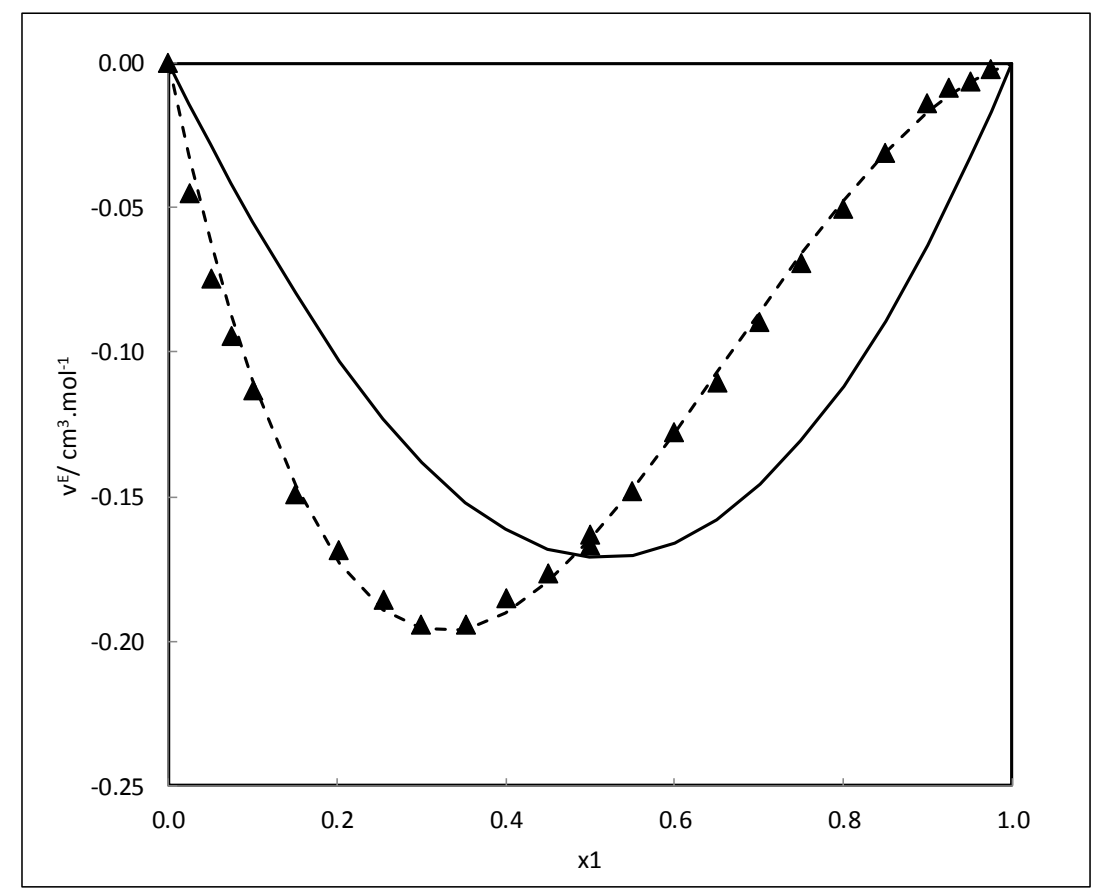

Figure S10 : Excess volumes for the furan(1) + Ethanol(2) binary system at $283 \mathrm{~K}$ as a function of molar composition. Symbols: experimental data, solid line: Calculated using PFP model with constant value of $\chi_{12}$, dashed line: Calculated with PFP model with constant $\chi_{12}$ composition dependent.

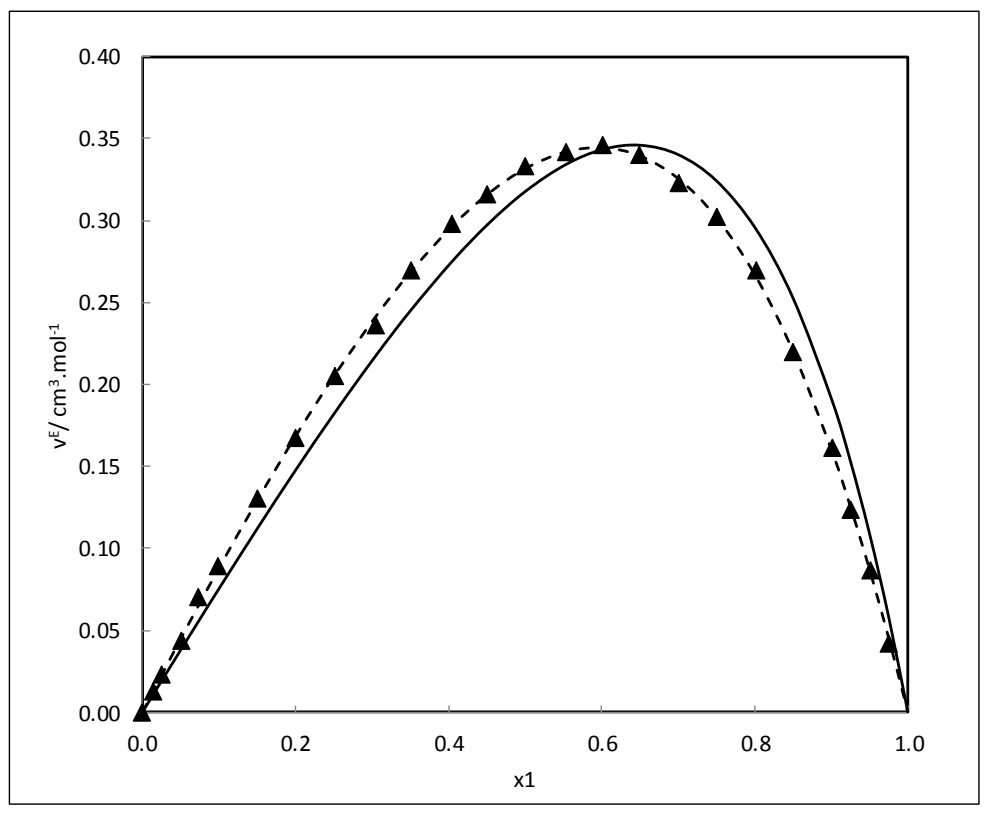

Figure S11: Excess volumes for the Furan(1) + 1-Octanol(2) binary system at $283 \mathrm{~K}$ as a function of molar composition. Symbols: experimental data, solid line: Calculated using PFP model with constant value of $\chi_{12}$, dashed line: Calculated with PFP model with constant $\chi_{12}$ composition dependent. 


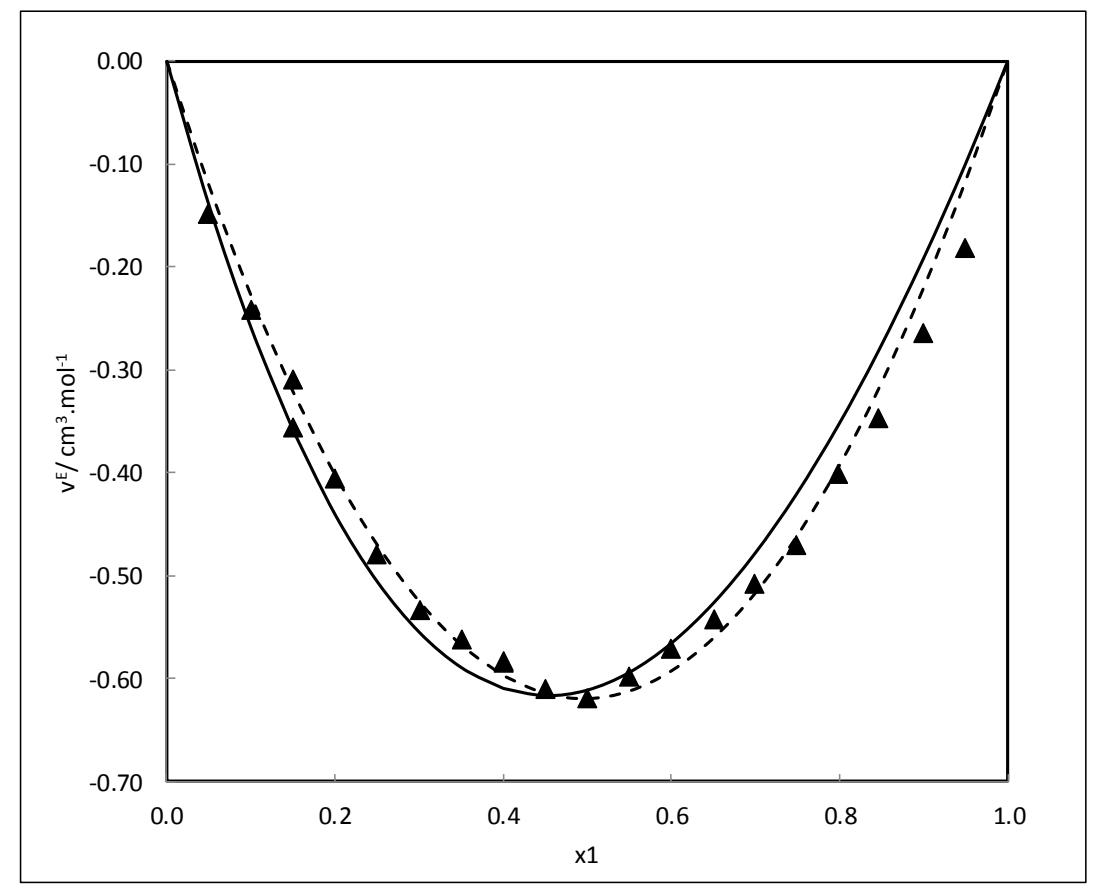

Figure S12 : Excess volumes for the Eugenol(1) + 1-Octanol(2) binary system at $283 \mathrm{~K}$ as a function of molar composition. Symbols: experimental data, solid line: Calculated using PFP model with constant value of $\chi_{12}$, dashed line: Calculated with PFP model with constant $\chi_{12}$ composition dependent.

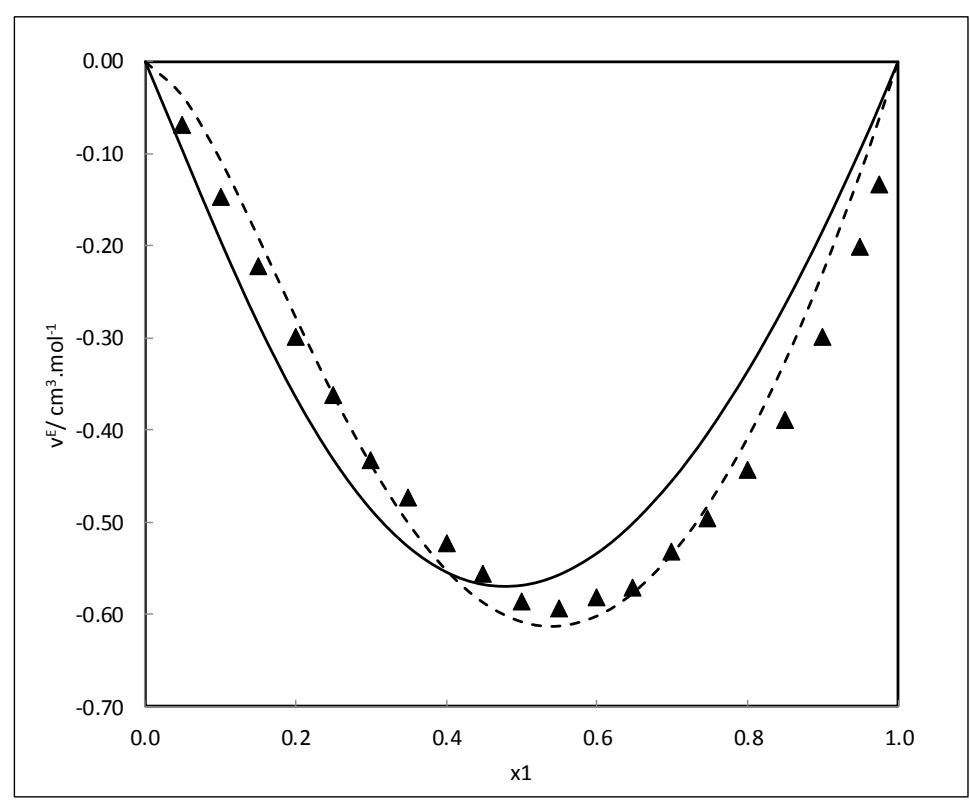

Figure S13 : Excess volumes for the Eugenol(1) $+n$-hexane(2) binary system at $283 \mathrm{~K}$ as a function of molar composition. Symbols: experimental data, solid line: Calculated using PFP model with constant value of $\chi_{12}$, dashed line: Calculated with PFP model with $\chi_{12}$ composition dependent. 
Table S2: Evaluation of the different contribution of the PFP model for the furan(1) + ethanol(2) binary system at $298.15 \mathrm{~K}$ and comparison with experimental data.

\begin{tabular}{|c|c|c|c|c|c|c|c|c|c|c|c|}
\hline \multicolumn{2}{|c|}{ Experimental data } & \multicolumn{5}{|c|}{ Constant value of $\chi_{12}$} & \multicolumn{5}{|c|}{$\chi_{12}$ composition dependent } \\
\hline \multirow{2}{*}{$\mathbf{x}_{1}$} & \multirow{2}{*}{$\mathbf{v}^{\mathrm{E}} / \mathrm{cm}^{3} \cdot \mathrm{mol}^{-1}$} & \multicolumn{3}{|c|}{ Calculated contribution $/ \mathrm{cm}^{3} \cdot \mathrm{mol}^{-1}$} & \multirow{2}{*}{$\begin{array}{c}\mathbf{v}^{\mathrm{E}} / \\
\mathrm{cm}^{3} \cdot \mathrm{mol}^{-1}\end{array}$} & \multirow{2}{*}{$\begin{array}{c}\text { Deviation / } \\
\mathrm{cm}^{3} \cdot \mathrm{mol}^{-1}\end{array}$} & \multicolumn{3}{|c|}{ Calculated contribution $/ \mathrm{cm}^{3} \cdot \mathrm{mol}^{-1}$} & \multirow{2}{*}{$\begin{array}{c}\mathrm{v}^{\mathrm{E}} / \\
\mathrm{cm}^{3} \cdot \mathrm{mol}^{-1}\end{array}$} & \multirow{2}{*}{$\begin{array}{l}\text { Deviation } \\
/ \mathrm{cm}^{3} \cdot \mathrm{mol}^{-1}\end{array}$} \\
\hline & & Interactional & Free volume & P* effect & & & Interactional & Free volume & P* effect & & \\
\hline 0.0248 & -0.044 & -0.034 & -0.014 & 0.037 & -0.011 & -0.033 & -0.054 & -0.014 & 0.037 & -0.030 & -0.014 \\
\hline 0.0501 & -0.074 & -0.067 & -0.026 & 0.071 & -0.022 & -0.052 & -0.103 & -0.026 & 0.072 & -0.058 & -0.016 \\
\hline 0.0748 & -0.090 & -0.097 & -0.037 & 0.102 & -0.032 & -0.058 & -0.147 & -0.037 & 0.102 & -0.082 & -0.008 \\
\hline 0.1005 & -0.107 & -0.127 & -0.047 & 0.132 & -0.043 & -0.064 & -0.188 & -0.047 & 0.132 & -0.103 & -0.003 \\
\hline 0.1505 & -0.137 & -0.179 & -0.063 & 0.181 & -0.062 & -0.075 & -0.254 & -0.063 & 0.181 & -0.136 & -0.001 \\
\hline 0.2027 & -0.155 & -0.226 & -0.076 & 0.221 & -0.081 & -0.074 & -0.305 & -0.076 & 0.222 & -0.159 & 0.004 \\
\hline 0.2549 & -0.168 & -0.265 & -0.085 & 0.253 & -0.098 & -0.070 & -0.340 & -0.085 & 0.253 & -0.172 & 0.004 \\
\hline 0.2999 & -0.172 & -0.293 & -0.090 & 0.273 & -0.110 & -0.062 & -0.359 & -0.090 & 0.273 & -0.176 & 0.004 \\
\hline 0.3526 & -0.170 & -0.317 & -0.093 & 0.288 & -0.122 & -0.048 & -0.368 & -0.093 & 0.289 & -0.173 & 0.002 \\
\hline 0.3999 & -0.159 & -0.332 & -0.094 & 0.296 & -0.130 & -0.029 & -0.366 & -0.094 & 0.296 & -0.164 & 0.005 \\
\hline 0.4500 & -0.148 & -0.341 & -0.093 & 0.297 & -0.136 & -0.012 & -0.356 & -0.093 & 0.298 & -0.151 & 0.003 \\
\hline 0.4998 & -0.134 & -0.342 & -0.090 & 0.293 & -0.139 & 0.005 & -0.338 & -0.090 & 0.293 & -0.134 & 0.001 \\
\hline 0.4998 & -0.137 & -0.342 & -0.090 & 0.293 & -0.139 & 0.002 & -0.338 & -0.090 & 0.293 & -0.134 & -0.003 \\
\hline 0.5499 & -0.117 & -0.337 & -0.086 & 0.283 & -0.140 & 0.022 & -0.313 & -0.086 & 0.283 & -0.115 & -0.002 \\
\hline 0.6000 & -0.096 & -0.325 & -0.080 & 0.268 & -0.137 & 0.040 & -0.284 & -0.080 & 0.268 & -0.095 & -0.001 \\
\hline 0.6500 & -0.079 & -0.306 & -0.073 & 0.248 & -0.131 & 0.052 & -0.250 & -0.073 & 0.249 & -0.074 & -0.005 \\
\hline 0.7009 & -0.058 & -0.280 & -0.064 & 0.224 & -0.121 & 0.063 & -0.214 & -0.064 & 0.224 & -0.054 & -0.004 \\
\hline 0.7500 & -0.039 & -0.249 & -0.056 & 0.196 & -0.109 & 0.070 & -0.177 & -0.056 & 0.196 & -0.036 & -0.003 \\
\hline 0.8000 & -0.023 & -0.211 & -0.046 & 0.164 & -0.094 & 0.070 & -0.139 & -0.046 & 0.164 & -0.021 & -0.002 \\
\hline 0.8499 & -0.007 & -0.167 & -0.035 & 0.128 & -0.075 & 0.068 & -0.101 & -0.035 & 0.128 & -0.009 & 0.002 \\
\hline 0.9000 & 0.005 & -0.117 & -0.024 & 0.088 & -0.053 & 0.058 & -0.065 & -0.024 & 0.088 & 0.000 & 0.006 \\
\hline 0.9250 & 0.007 & -0.090 & -0.018 & 0.067 & -0.041 & 0.048 & -0.047 & -0.018 & 0.067 & 0.002 & 0.006 \\
\hline 0.9500 & 0.006 & -0.061 & -0.012 & 0.046 & -0.028 & 0.034 & -0.031 & -0.012 & 0.046 & 0.003 & 0.004 \\
\hline
\end{tabular}


Table S3: Evaluation of the different contribution of the PFP model for the furan(1) + 1-Octanol(2) binary system at $298.15 \mathrm{~K}$ and comparison with experimental data.

\begin{tabular}{|c|c|c|c|c|c|c|c|c|c|c|c|}
\hline \multicolumn{2}{|c|}{ Experimental data } & \multicolumn{5}{|c|}{ Constant value of $\chi_{12}$} & \multicolumn{5}{|c|}{$\chi_{12}$ composition dependent } \\
\hline \multirow{2}{*}{$\mathbf{x}_{1}$} & \multirow{2}{*}{$\mathbf{v}^{\mathrm{E}} / \mathbf{c m}^{3} \cdot \mathbf{m o l}^{-1}$} & \multicolumn{3}{|c|}{ Calculated contribution $/ \mathrm{cm}^{3} \cdot \mathrm{mol}^{-1}$} & \multirow{2}{*}{$\begin{array}{c}\mathbf{v}^{\mathrm{E}} / \\
\mathrm{cm}^{3} \cdot \mathrm{mol}^{-}\end{array}$} & \multirow{2}{*}{$\begin{array}{l}\text { Deviation } \\
/ \mathrm{cm}^{3} \cdot \mathrm{mol}^{-1}\end{array}$} & \multicolumn{3}{|c|}{ Calculated contribution $/ \mathrm{cm}^{3} \cdot \mathrm{mol}^{-1}$} & \multirow[t]{2}{*}{$\mathbf{v}^{\mathrm{E}} / \mathbf{c m}^{3} \cdot \mathrm{mol}^{-}$} & \multirow{2}{*}{$\begin{array}{l}\text { Deviation } \\
/ \mathrm{cm}_{1}^{3} . \mathrm{mol}\end{array}$} \\
\hline & & Interactional & Free volume & P* effect & & & Interactional & Free volume & P* effect & & \\
\hline 0.0134 & 0.010 & 0.001 & -0.021 & 0.031 & 0.011 & -0.001 & 0.002 & -0.021 & 0.031 & 0.012 & -0.002 \\
\hline 0.0256 & 0.022 & 0.002 & -0.041 & 0.060 & 0.021 & 0.000 & 0.003 & -0.041 & 0.060 & 0.022 & -0.001 \\
\hline 0.0506 & 0.045 & 0.004 & -0.079 & 0.116 & 0.042 & 0.003 & 0.006 & -0.079 & 0.116 & 0.044 & 0.001 \\
\hline 0.0739 & 0.067 & 0.005 & -0.113 & 0.168 & 0.060 & 0.007 & 0.008 & -0.113 & 0.168 & 0.063 & 0.004 \\
\hline 0.0995 & 0.089 & 0.007 & -0.149 & 0.222 & 0.081 & 0.008 & 0.011 & -0.149 & 0.222 & 0.085 & 0.004 \\
\hline 0.1495 & 0.132 & 0.011 & -0.213 & 0.322 & 0.119 & 0.013 & 0.016 & -0.213 & 0.322 & 0.125 & 0.007 \\
\hline 0.1999 & 0.165 & 0.014 & -0.272 & 0.415 & 0.157 & 0.008 & 0.021 & -0.272 & 0.415 & 0.164 & 0.001 \\
\hline 0.2512 & 0.209 & 0.017 & -0.324 & 0.500 & 0.193 & 0.016 & 0.025 & -0.324 & 0.500 & 0.201 & 0.008 \\
\hline 0.3042 & 0.241 & 0.020 & -0.370 & 0.578 & 0.228 & 0.014 & 0.029 & -0.370 & 0.578 & 0.237 & 0.005 \\
\hline 0.3501 & 0.270 & 0.022 & -0.403 & 0.636 & 0.255 & 0.015 & 0.032 & -0.403 & 0.636 & 0.265 & 0.006 \\
\hline 0.4043 & 0.302 & 0.025 & -0.432 & 0.692 & 0.285 & 0.017 & 0.034 & -0.432 & 0.692 & 0.294 & 0.008 \\
\hline 0.4511 & 0.321 & 0.027 & -0.449 & 0.729 & 0.306 & 0.015 & 0.035 & -0.449 & 0.729 & 0.315 & 0.006 \\
\hline 0.5000 & 0.334 & 0.028 & -0.459 & 0.756 & 0.325 & 0.009 & 0.036 & -0.459 & 0.756 & 0.333 & 0.001 \\
\hline 0.5529 & 0.345 & 0.029 & -0.460 & 0.770 & 0.339 & 0.006 & 0.036 & -0.460 & 0.770 & 0.346 & -0.001 \\
\hline 0.6016 & 0.349 & 0.030 & -0.451 & 0.768 & 0.346 & 0.003 & 0.035 & -0.451 & 0.768 & 0.352 & -0.003 \\
\hline 0.6500 & 0.343 & 0.029 & -0.433 & 0.750 & 0.346 & -0.003 & 0.034 & -0.433 & 0.750 & 0.350 & -0.007 \\
\hline 0.7000 & 0.322 & 0.028 & -0.405 & 0.714 & 0.338 & -0.015 & 0.031 & -0.405 & 0.714 & 0.340 & -0.018 \\
\hline 0.7499 & 0.303 & 0.027 & -0.365 & 0.658 & 0.319 & -0.016 & 0.027 & -0.365 & 0.658 & 0.320 & -0.017 \\
\hline 0.8010 & 0.271 & 0.024 & -0.314 & 0.578 & 0.288 & -0.017 & 0.023 & -0.314 & 0.578 & 0.287 & -0.016 \\
\hline 0.8500 & 0.220 & 0.020 & -0.253 & 0.478 & 0.245 & -0.025 & 0.017 & -0.253 & 0.478 & 0.242 & -0.022 \\
\hline 0.9010 & 0.163 & 0.015 & -0.179 & 0.346 & 0.183 & -0.019 & 0.011 & -0.179 & 0.346 & 0.179 & -0.016 \\
\hline 0.9250 & 0.125 & 0.012 & -0.139 & 0.274 & 0.147 & -0.022 & 0.009 & -0.139 & 0.274 & 0.143 & -0.018 \\
\hline 0.9500 & 0.089 & 0.008 & -0.096 & 0.191 & 0.104 & -0.015 & 0.006 & -0.096 & 0.191 & 0.101 & -0.012 \\
\hline 0.9750 & 0.044 & 0.004 & -0.049 & 0.100 & 0.055 & -0.011 & 0.003 & -0.049 & 0.100 & 0.053 & -0.010 \\
\hline
\end{tabular}


Table S4: Evaluation of the different contribution of the PFP model for the Eugenol(1) + 1-Octanol(2) binary system at $298.15 \mathrm{~K}$ and comparison with experimental data.

\begin{tabular}{|c|c|c|c|c|c|c|c|c|c|c|c|}
\hline \multicolumn{2}{|c|}{ Experimental data } & \multicolumn{5}{|c|}{ Constant value of $\chi_{12}$} & \multicolumn{5}{|c|}{$\chi_{12}$ composition dependent } \\
\hline \multirow[b]{2}{*}{$\mathbf{x}_{1}$} & \multirow{2}{*}{$\mathbf{v}^{\mathrm{E}} / \mathrm{cm}^{3} \cdot \mathrm{mol}^{-1}$} & \multicolumn{3}{|c|}{ Calculated contribution $/ \mathrm{cm}^{3} \cdot \mathrm{mol}^{-1}$} & \multirow{2}{*}{$\begin{array}{c}\mathbf{v}^{\mathrm{E}} / \\
\mathrm{cm}^{3} \cdot \mathrm{mol}^{-1}\end{array}$} & \multirow{2}{*}{$\begin{array}{l}\text { Deviation } \\
/ \mathrm{cm}^{3} \cdot \mathrm{mol}^{-1}\end{array}$} & \multicolumn{3}{|c|}{ Calculated contribution $/ \mathrm{cm}^{3} \cdot \mathrm{mol}^{-1}$} & \multirow{2}{*}{$\begin{array}{c}\mathbf{v}^{\mathrm{E} /} \\
\mathrm{cm}^{3} \cdot \mathrm{mol}^{-1}\end{array}$} & \multirow{2}{*}{$\begin{array}{c}\text { Deviation } \\
/ \\
\mathrm{cm}^{3} \cdot \mathrm{mol}^{-1} \\
\end{array}$} \\
\hline & & Interactional & Free volume & $\mathbf{P} *$ effect & & & Interactional & Free volume & P* effect & & \\
\hline 0.1001 & -0.215 & -0.214 & -0.0002 & -0.016 & -0.230 & 0.015 & -0.184 & -0.0002 & -0.016 & -0.200 & -0.015 \\
\hline 0.1500 & -0.318 & -0.297 & -0.0003 & -0.022 & -0.319 & 0.001 & -0.260 & -0.0003 & -0.022 & -0.282 & -0.036 \\
\hline 0.1502 & -0.273 & -0.297 & -0.0003 & -0.022 & -0.319 & 0.046 & -0.261 & -0.0003 & -0.022 & -0.283 & 0.010 \\
\hline 0.2502 & -0.425 & -0.418 & -0.0004 & -0.031 & -0.449 & 0.025 & -0.383 & -0.0004 & -0.031 & -0.415 & -0.010 \\
\hline 0.3004 & -0.471 & -0.459 & -0.0004 & -0.034 & -0.493 & 0.022 & -0.430 & -0.0004 & -0.034 & -0.464 & -0.007 \\
\hline 0.3502 & -0.495 & -0.487 & -0.0005 & -0.036 & -0.523 & 0.028 & -0.466 & -0.0005 & -0.036 & -0.502 & 0.007 \\
\hline 0.4001 & -0.516 & -0.503 & -0.0005 & -0.037 & -0.541 & 0.025 & -0.492 & -0.0005 & -0.037 & -0.529 & 0.013 \\
\hline 0.4003 & -0.513 & -0.503 & -0.0005 & -0.037 & -0.541 & 0.028 & -0.492 & -0.0005 & -0.037 & -0.529 & 0.016 \\
\hline 0.4501 & -0.539 & -0.509 & -0.0005 & -0.038 & -0.547 & 0.008 & -0.507 & -0.0005 & -0.038 & -0.545 & 0.006 \\
\hline 0.5002 & -0.552 & -0.504 & -0.0004 & -0.037 & -0.542 & -0.009 & -0.513 & -0.0004 & -0.037 & -0.551 & -0.001 \\
\hline 0.6000 & -0.506 & -0.466 & -0.0004 & -0.035 & -0.501 & -0.005 & -0.493 & -0.0004 & -0.035 & -0.528 & 0.022 \\
\hline 0.6502 & -0.482 & -0.434 & -0.0004 & -0.032 & -0.467 & -0.015 & -0.467 & -0.0004 & -0.032 & -0.500 & 0.018 \\
\hline 0.6992 & -0.455 & -0.394 & -0.0003 & -0.029 & -0.424 & -0.031 & -0.432 & -0.0003 & -0.029 & -0.462 & 0.007 \\
\hline 0.7482 & -0.420 & -0.347 & -0.0003 & -0.026 & -0.373 & -0.047 & -0.388 & -0.0003 & -0.026 & -0.414 & -0.007 \\
\hline 0.7984 & -0.358 & -0.292 & -0.0002 & -0.022 & -0.314 & -0.045 & -0.331 & -0.0002 & -0.022 & -0.353 & -0.005 \\
\hline 0.8457 & -0.316 & -0.233 & -0.0002 & -0.017 & -0.250 & -0.066 & -0.269 & -0.0002 & -0.017 & -0.286 & -0.029 \\
\hline 0.8997 & -0.241 & -0.158 & -0.0001 & -0.012 & -0.170 & -0.071 & -0.186 & -0.0001 & -0.012 & -0.198 & -0.043 \\
\hline 0.9499 & -0.171 & -0.082 & -0.0001 & -0.006 & -0.088 & -0.083 & -0.098 & -0.0001 & -0.006 & -0.104 & -0.067 \\
\hline
\end{tabular}


Table S5: Evaluation of the different contribution of the PFP model for the Eugenol(1) + $\mathrm{n}$-hexane(2) binary system at $298.15 \mathrm{~K}$ and comparison with experimental data.

\begin{tabular}{|c|c|c|c|c|c|c|c|c|c|c|c|}
\hline \multicolumn{2}{|c|}{ Experimental data } & \multicolumn{5}{|c|}{ Constant value of $\chi_{12}$} & \multicolumn{5}{|c|}{$\chi_{12}$ composition dependent } \\
\hline \multirow[b]{2}{*}{$\mathbf{x}_{1}$} & \multirow{2}{*}{$\mathbf{v}^{\mathrm{E}} / \mathbf{c m}^{3} \cdot \mathbf{m o l}^{-1}$} & \multicolumn{3}{|c|}{ Calculated contribution $/ \mathrm{cm}^{3} . \mathrm{mol}^{-1}$} & \multirow{2}{*}{$\begin{array}{c}\mathbf{v}^{\mathrm{E}} / \\
\mathrm{cm}^{3} \cdot \mathrm{mol}^{-1}\end{array}$} & \multirow{2}{*}{$\begin{array}{l}\text { Deviation } \\
/ \mathbf{c m}^{3} \cdot \mathbf{m o l}^{-1}\end{array}$} & \multicolumn{3}{|c|}{ Calculated contribution $/ \mathrm{cm}^{3} \cdot \mathrm{mol}^{-1}$} & \multirow{2}{*}{$\begin{array}{c}\mathbf{v}^{\mathrm{E}} / \\
\mathrm{cm}^{3} \cdot \mathrm{mol}^{-1}\end{array}$} & \multirow{2}{*}{$\begin{array}{c}\text { Deviation } \\
/ \\
\mathrm{cm}^{3} \cdot \mathrm{mol}^{-1}\end{array}$} \\
\hline & & Interactional & Free volume & $\mathbf{P} *$ effect & & & Interactional & Free volume & P* effect & & \\
\hline 0.1000 & -0.207 & 0.770 & -0.284 & -0.726 & -0.240 & 0.034 & 0.848 & -0.284 & -0.726 & -0.162 & -0.045 \\
\hline 0.1500 & -0.301 & 0.998 & -0.371 & -0.981 & -0.353 & 0.052 & 1.082 & -0.371 & -0.981 & -0.269 & -0.032 \\
\hline 0.2000 & -0.397 & 1.153 & -0.431 & -1.175 & -0.453 & 0.057 & 1.229 & -0.431 & -1.175 & -0.377 & -0.020 \\
\hline 0.3000 & -0.556 & 1.299 & -0.491 & -1.415 & -0.607 & 0.050 & 1.342 & -0.491 & -1.415 & -0.564 & 0.008 \\
\hline 0.3500 & -0.609 & 1.312 & -0.497 & -1.472 & -0.657 & 0.048 & 1.334 & -0.497 & -1.472 & -0.636 & 0.027 \\
\hline 0.4000 & -0.659 & 1.295 & -0.493 & -1.494 & -0.691 & 0.033 & 1.296 & -0.493 & -1.494 & -0.690 & 0.032 \\
\hline 0.4491 & -0.692 & 1.255 & -0.478 & -1.485 & -0.708 & 0.016 & 1.237 & -0.478 & -1.485 & -0.726 & 0.033 \\
\hline 0.5000 & -0.722 & 1.193 & -0.455 & -1.446 & -0.709 & -0.013 & 1.157 & -0.455 & -1.446 & -0.744 & 0.022 \\
\hline 0.5489 & -0.723 & 1.117 & -0.427 & -1.384 & -0.695 & -0.028 & 1.068 & -0.427 & -1.384 & -0.744 & 0.021 \\
\hline 0.6000 & -0.703 & 1.023 & -0.392 & -1.296 & -0.665 & -0.038 & 0.963 & -0.392 & -1.296 & -0.725 & 0.021 \\
\hline 0.6994 & -0.635 & 0.809 & -0.310 & -1.066 & -0.567 & -0.069 & 0.740 & -0.310 & -1.066 & -0.636 & 0.001 \\
\hline 0.7464 & -0.587 & 0.696 & -0.267 & -0.932 & -0.503 & -0.084 & 0.627 & -0.267 & -0.932 & -0.571 & -0.016 \\
\hline 0.8000 & -0.520 & 0.560 & -0.215 & -0.763 & -0.418 & -0.102 & 0.497 & -0.215 & -0.763 & -0.481 & -0.039 \\
\hline 0.8499 & -0.449 & 0.426 & -0.164 & -0.591 & -0.328 & -0.120 & 0.373 & -0.164 & -0.591 & -0.381 & -0.067 \\
\hline 0.8991 & -0.341 & 0.291 & -0.111 & -0.409 & -0.230 & -0.112 & 0.251 & -0.111 & -0.409 & -0.270 & -0.072 \\
\hline 0.9498 & -0.226 & 0.146 & -0.056 & -0.209 & -0.119 & -0.107 & 0.125 & -0.056 & -0.209 & -0.141 & -0.085 \\
\hline 0.9751 & -0.146 & 0.073 & -0.028 & -0.105 & -0.060 & -0.086 & 0.062 & -0.028 & -0.105 & -0.071 & -0.075 \\
\hline
\end{tabular}


Table S6: Evaluation of the different contribution of the PFP model for the furan(1) + ethanol(2) binary system at $283.15 \mathrm{~K}$ and comparison with experimental data.

\begin{tabular}{|c|c|c|c|c|c|c|c|c|c|c|c|}
\hline \multicolumn{2}{|c|}{ Experimental data } & \multicolumn{5}{|c|}{ Constant value of $\chi_{12}$} & \multicolumn{5}{|c|}{$\chi_{12}$ composition dependent } \\
\hline \multirow{2}{*}{$\mathbf{x}_{1}$} & \multirow{2}{*}{$\mathrm{v}^{\mathrm{E}} / \mathrm{cm}^{3} \cdot \mathrm{mol}^{-1}$} & \multicolumn{3}{|c|}{ Calculated contribution $/ \mathrm{cm}^{3} \cdot \mathrm{mol}^{-1}$} & \multirow{2}{*}{$\begin{array}{c}\mathbf{v}^{\mathrm{E}} / \\
\mathrm{cm}^{3} \cdot \mathrm{mol}^{-1}\end{array}$} & \multirow{2}{*}{$\begin{array}{c}\text { Deviation / } \\
\mathrm{cm}^{3} \cdot \mathrm{mol}^{-1}\end{array}$} & \multicolumn{3}{|c|}{ Calculated contribution $/ \mathrm{cm}^{3} \cdot \mathrm{mol}^{-1}$} & \multirow{2}{*}{$\begin{array}{c}\mathrm{v}^{\mathrm{E}} / \\
\mathrm{cm}^{3} \cdot \mathrm{mol}^{-1}\end{array}$} & \multirow{2}{*}{$\begin{array}{c}\text { Deviation } \\
/ \mathrm{cm}^{3} \cdot \mathrm{mol}^{-1} \\
\end{array}$} \\
\hline & & Interactional & Free volume & P* effect & & & Interactional & Free volume & P* effect & & \\
\hline 0.0248 & -0.045 & -0.038 & -0.011 & 0.035 & -0.014 & -0.031 & -0.056 & -0.011 & 0.035 & -0.032 & -0.013 \\
\hline 0.0501 & -0.075 & -0.075 & -0.021 & 0.068 & -0.028 & -0.047 & -0.108 & -0.021 & 0.068 & -0.061 & -0.013 \\
\hline 0.0748 & -0.095 & -0.109 & -0.030 & 0.098 & -0.042 & -0.053 & -0.154 & -0.030 & 0.098 & -0.087 & -0.008 \\
\hline 0.1005 & -0.113 & -0.142 & -0.038 & 0.125 & -0.055 & -0.058 & -0.197 & -0.038 & 0.125 & -0.110 & -0.003 \\
\hline 0.1505 & -0.149 & -0.200 & -0.051 & 0.172 & -0.080 & -0.069 & -0.267 & -0.051 & 0.172 & -0.146 & -0.003 \\
\hline 0.2027 & -0.169 & -0.252 & -0.061 & 0.210 & -0.103 & -0.066 & -0.321 & -0.061 & 0.210 & -0.173 & 0.004 \\
\hline 0.2549 & -0.186 & -0.294 & -0.068 & 0.239 & -0.123 & -0.063 & -0.360 & -0.068 & 0.239 & -0.189 & 0.003 \\
\hline 0.2999 & -0.194 & -0.323 & -0.072 & 0.258 & -0.138 & -0.056 & -0.381 & -0.072 & 0.258 & -0.196 & 0.002 \\
\hline 0.3526 & -0.194 & -0.349 & -0.075 & 0.272 & -0.152 & -0.042 & -0.393 & -0.075 & 0.272 & -0.196 & 0.002 \\
\hline 0.3999 & -0.185 & -0.365 & -0.075 & 0.278 & -0.161 & -0.023 & -0.394 & -0.075 & 0.278 & -0.190 & 0.006 \\
\hline 0.4500 & -0.177 & -0.373 & -0.074 & 0.279 & -0.168 & -0.009 & -0.385 & -0.074 & 0.279 & -0.180 & 0.003 \\
\hline 0.4998 & -0.163 & -0.374 & -0.072 & 0.275 & -0.171 & 0.008 & -0.369 & -0.072 & 0.275 & -0.165 & 0.002 \\
\hline 0.4998 & -0.167 & -0.374 & -0.072 & 0.275 & -0.171 & 0.004 & -0.369 & -0.072 & 0.275 & -0.165 & -0.002 \\
\hline 0.6000 & -0.128 & -0.354 & -0.063 & 0.251 & -0.166 & 0.038 & -0.316 & -0.063 & 0.251 & -0.128 & 0.000 \\
\hline 0.6500 & -0.111 & -0.332 & -0.057 & 0.232 & -0.158 & 0.047 & -0.282 & -0.057 & 0.232 & -0.107 & -0.003 \\
\hline 0.7009 & -0.090 & -0.304 & -0.051 & 0.209 & -0.146 & 0.056 & -0.244 & -0.051 & 0.209 & -0.086 & -0.004 \\
\hline 0.7500 & -0.069 & -0.269 & -0.044 & 0.183 & -0.131 & 0.061 & -0.205 & -0.044 & 0.183 & -0.066 & -0.003 \\
\hline 0.8000 & -0.050 & -0.228 & -0.036 & 0.152 & -0.112 & 0.061 & -0.164 & -0.036 & 0.152 & -0.048 & -0.003 \\
\hline 0.8499 & -0.031 & -0.180 & -0.028 & 0.119 & -0.089 & 0.058 & -0.122 & -0.028 & 0.119 & -0.031 & 0.000 \\
\hline 0.9000 & -0.014 & -0.126 & -0.019 & 0.082 & -0.063 & 0.049 & -0.080 & -0.019 & 0.082 & -0.017 & 0.003 \\
\hline 0.9250 & -0.009 & -0.097 & -0.014 & 0.063 & -0.048 & 0.040 & -0.060 & -0.014 & 0.063 & -0.011 & 0.003 \\
\hline 0.9500 & -0.007 & -0.066 & -0.010 & 0.042 & -0.033 & 0.027 & -0.039 & -0.010 & 0.042 & -0.007 & 0.000 \\
\hline
\end{tabular}


Table S7: Evaluation of the different contribution of the PFP model for the furan(1) + 1-Octanol(2) binary system at $283.15 \mathrm{~K}$ and comparison with experimental data.

\begin{tabular}{|c|c|c|c|c|c|c|c|c|c|c|c|}
\hline \multicolumn{2}{|c|}{ Experimental data } & \multicolumn{5}{|c|}{ Constant value of $\chi_{12}$} & \multicolumn{5}{|c|}{$\chi_{12}$ composition dependent } \\
\hline \multirow{2}{*}{$\mathbf{x}_{1}$} & \multirow{2}{*}{$\mathrm{v}^{\mathrm{E}} / \mathrm{cm}^{3} \cdot \mathrm{mol}^{-1}$} & \multicolumn{3}{|c|}{ Calculated contribution $/ \mathrm{cm}^{3} \cdot \mathrm{mol}^{-1}$} & \multirow{2}{*}{$\begin{array}{c}\mathbf{v}^{\mathrm{E}} / \\
\mathrm{cm}^{3} \cdot \mathrm{mol}^{-1}\end{array}$} & \multirow{2}{*}{$\begin{array}{l}\text { Deviation } \\
/ \mathrm{cm}^{3} \cdot \mathrm{mol}^{-1}\end{array}$} & \multicolumn{3}{|c|}{ Calculated contribution $/ \mathrm{cm}^{3} \cdot \mathrm{mol}^{-1}$} & \multirow{2}{*}{$\begin{array}{c}\mathbf{v}^{\mathrm{E}} / \\
\mathrm{cm}^{3} \cdot \mathrm{mol}^{-1} \\
\end{array}$} & \multirow{2}{*}{$\begin{array}{l}\text { Deviation } \\
/ \mathrm{cm}^{3} . \mathrm{mol}^{-1} \\
\end{array}$} \\
\hline & & Interactional & Free volume & P* effect & & & Interactional & Free volume & $\mathbf{P} *$ effect & & \\
\hline 0.0134 & 0.013 & 0.005 & -0.021 & 0.026 & 0.010 & 0.003 & 0.007 & -0.021 & 0.026 & 0.012 & 0.001 \\
\hline 0.0256 & 0.023 & 0.010 & -0.040 & 0.049 & 0.019 & 0.003 & 0.014 & -0.040 & 0.049 & 0.023 & 0.000 \\
\hline 0.0506 & 0.043 & 0.020 & -0.077 & 0.095 & 0.038 & 0.005 & 0.027 & -0.077 & 0.095 & 0.045 & -0.002 \\
\hline 0.0739 & 0.070 & 0.029 & -0.110 & 0.137 & 0.056 & 0.014 & 0.039 & -0.110 & 0.137 & 0.066 & 0.004 \\
\hline 0.0995 & 0.089 & 0.039 & -0.146 & 0.181 & 0.075 & 0.014 & 0.052 & -0.146 & 0.181 & 0.088 & 0.002 \\
\hline 0.1495 & 0.130 & 0.058 & -0.210 & 0.263 & 0.112 & 0.019 & 0.075 & -0.210 & 0.263 & 0.129 & 0.002 \\
\hline 0.1999 & 0.167 & 0.076 & -0.268 & 0.340 & 0.148 & 0.020 & 0.097 & -0.268 & 0.340 & 0.168 & -0.001 \\
\hline 0.2512 & 0.205 & 0.093 & -0.321 & 0.410 & 0.183 & 0.022 & 0.116 & -0.321 & 0.410 & 0.206 & -0.001 \\
\hline 0.3042 & 0.236 & 0.110 & -0.367 & 0.475 & 0.217 & 0.019 & 0.134 & -0.367 & 0.475 & 0.241 & -0.005 \\
\hline 0.3501 & 0.269 & 0.123 & -0.401 & 0.523 & 0.245 & 0.024 & 0.147 & -0.401 & 0.523 & 0.269 & 0.001 \\
\hline 0.4043 & 0.298 & 0.137 & -0.432 & 0.570 & 0.275 & 0.023 & 0.158 & -0.432 & 0.570 & 0.297 & 0.001 \\
\hline 0.4511 & 0.316 & 0.147 & -0.451 & 0.602 & 0.298 & 0.018 & 0.165 & -0.451 & 0.602 & 0.316 & 0.000 \\
\hline 0.5000 & 0.333 & 0.156 & -0.463 & 0.625 & 0.318 & 0.015 & 0.170 & -0.463 & 0.625 & 0.332 & 0.001 \\
\hline 0.6016 & 0.346 & 0.165 & -0.459 & 0.638 & 0.344 & 0.003 & 0.166 & -0.459 & 0.638 & 0.345 & 0.002 \\
\hline 0.6500 & 0.340 & 0.165 & -0.443 & 0.625 & 0.346 & -0.006 & 0.158 & -0.443 & 0.625 & 0.340 & 0.001 \\
\hline 0.7000 & 0.323 & 0.161 & -0.416 & 0.596 & 0.340 & -0.018 & 0.146 & -0.416 & 0.596 & 0.326 & -0.003 \\
\hline 0.7499 & 0.302 & 0.152 & -0.378 & 0.551 & 0.324 & -0.023 & 0.129 & -0.378 & 0.551 & 0.302 & 0.000 \\
\hline 0.8010 & 0.269 & 0.137 & -0.326 & 0.485 & 0.295 & -0.026 & 0.108 & -0.326 & 0.485 & 0.266 & 0.003 \\
\hline 0.8500 & 0.220 & 0.116 & -0.265 & 0.402 & 0.253 & -0.033 & 0.083 & -0.265 & 0.402 & 0.220 & -0.001 \\
\hline 0.9010 & 0.161 & 0.086 & -0.188 & 0.293 & 0.190 & -0.029 & 0.055 & -0.188 & 0.293 & 0.159 & 0.002 \\
\hline 0.9250 & 0.123 & 0.069 & -0.148 & 0.232 & 0.153 & -0.030 & 0.041 & -0.148 & 0.232 & 0.125 & -0.002 \\
\hline 0.9500 & 0.086 & 0.049 & -0.102 & 0.162 & 0.109 & -0.023 & 0.027 & -0.102 & 0.162 & 0.087 & -0.001 \\
\hline 0.9750 & 0.042 & 0.026 & -0.053 & 0.085 & 0.058 & -0.016 & 0.013 & -0.053 & 0.085 & 0.045 & -0.003 \\
\hline
\end{tabular}


Table S8: Evaluation of the different contribution of the PFP model for the Eugenol(1) + 1-Octanol(2) binary system at $283.15 \mathrm{~K}$ and comparison with experimental data.

\begin{tabular}{|c|c|c|c|c|c|c|c|c|c|c|c|}
\hline \multicolumn{2}{|c|}{ Experimental data } & \multicolumn{5}{|c|}{ Constant value of $\chi_{12}$} & \multicolumn{5}{|c|}{$\chi_{12}$ composition dependent } \\
\hline \multirow[b]{2}{*}{$\mathbf{x}_{1}$} & \multirow{2}{*}{$\mathrm{v}^{\mathrm{E}} / \mathrm{cm}^{3} \cdot \mathrm{mol}^{-1}$} & \multicolumn{3}{|c|}{ Calculated contribution $/ \mathrm{cm}^{3} \cdot \mathrm{mol}^{-1}$} & \multirow{2}{*}{$\begin{array}{c}\mathbf{v}^{\mathrm{E}} / \\
\mathrm{cm}^{3} \cdot \mathrm{mol}^{-1}\end{array}$} & \multirow{2}{*}{$\begin{array}{l}\text { Deviation } \\
/ \mathrm{cm}^{3} \cdot \mathrm{mol}^{-1}\end{array}$} & \multicolumn{3}{|c|}{ Calculated contribution $/ \mathrm{cm}^{3} \cdot \mathrm{mol}^{-1}$} & \multirow{2}{*}{$\mathrm{cm}^{\mathbf{v}^{\mathrm{E}} / \mathrm{mol}^{-}}$} & \multirow{2}{*}{$\begin{array}{c}\text { Deviation } \\
/ \\
\mathrm{cm}^{3} \cdot \mathrm{mol}^{-1}\end{array}$} \\
\hline & & Interactional & Free volume & $P^{*}$ effect & & & Interactional & Free volume & P* effect & & \\
\hline 0.0500 & -0.149 & -0.137 & -0.00001 & -0.002 & -0.139 & -0.009 & -0.118 & -0.00001 & -0.002 & -0.120 & -0.028 \\
\hline 0.1001 & -0.242 & -0.254 & -0.00002 & -0.004 & -0.259 & 0.016 & -0.223 & -0.00002 & -0.004 & -0.227 & -0.015 \\
\hline 0.1500 & -0.356 & -0.352 & -0.00002 & -0.006 & -0.358 & 0.002 & -0.315 & -0.00002 & -0.006 & -0.321 & -0.035 \\
\hline 0.1502 & -0.310 & -0.352 & -0.00002 & -0.006 & -0.358 & 0.049 & -0.315 & -0.00002 & -0.006 & -0.321 & 0.012 \\
\hline 0.2002 & -0.406 & -0.433 & -0.00003 & -0.007 & -0.440 & 0.034 & -0.394 & -0.00003 & -0.007 & -0.402 & -0.004 \\
\hline 0.2502 & -0.479 & -0.497 & -0.00003 & -0.009 & -0.505 & 0.027 & -0.461 & -0.00003 & -0.009 & -0.470 & -0.009 \\
\hline 0.3004 & -0.534 & -0.545 & -0.00003 & -0.009 & -0.555 & 0.021 & -0.516 & -0.00003 & -0.009 & -0.525 & -0.009 \\
\hline 0.3502 & -0.562 & -0.579 & -0.00003 & -0.010 & -0.589 & 0.027 & -0.557 & -0.00003 & -0.010 & -0.567 & 0.006 \\
\hline 0.4001 & -0.585 & -0.599 & -0.00003 & -0.010 & -0.609 & 0.024 & -0.587 & -0.00003 & -0.010 & -0.597 & 0.012 \\
\hline 0.4003 & -0.583 & -0.599 & -0.00003 & -0.010 & -0.609 & 0.026 & -0.587 & -0.00003 & -0.010 & -0.597 & 0.014 \\
\hline 0.4501 & -0.610 & -0.606 & -0.00003 & -0.010 & -0.616 & 0.006 & -0.604 & -0.00003 & -0.010 & -0.614 & 0.004 \\
\hline 0.5002 & -0.619 & -0.600 & -0.00003 & -0.010 & -0.611 & -0.009 & -0.609 & -0.00003 & -0.010 & -0.619 & 0.000 \\
\hline 0.5502 & -0.598 & -0.583 & -0.00003 & -0.010 & -0.594 & -0.004 & -0.602 & -0.00003 & -0.010 & -0.612 & 0.014 \\
\hline 0.6000 & -0.571 & -0.556 & -0.00003 & -0.010 & -0.565 & -0.005 & -0.583 & -0.00003 & -0.010 & -0.592 & 0.022 \\
\hline 0.6502 & -0.543 & -0.517 & -0.00003 & -0.009 & -0.526 & -0.017 & -0.551 & -0.00003 & -0.009 & -0.560 & 0.017 \\
\hline 0.6992 & -0.509 & -0.470 & -0.00002 & -0.008 & -0.478 & -0.030 & -0.509 & -0.00002 & -0.008 & -0.517 & 0.009 \\
\hline 0.7482 & -0.470 & -0.414 & -0.00002 & -0.007 & -0.421 & -0.049 & -0.456 & -0.00002 & -0.007 & -0.463 & -0.007 \\
\hline 0.7984 & -0.401 & -0.348 & -0.00002 & -0.006 & -0.354 & -0.047 & -0.389 & -0.00002 & -0.006 & -0.395 & -0.006 \\
\hline 0.8457 & -0.347 & -0.277 & -0.00001 & -0.005 & -0.282 & -0.065 & -0.315 & -0.00001 & -0.005 & -0.320 & -0.027 \\
\hline 0.8997 & -0.265 & -0.189 & -0.00001 & -0.003 & -0.192 & -0.073 & -0.217 & -0.00001 & -0.003 & -0.221 & -0.044 \\
\hline 0.9499 & -0.182 & -0.098 & 0.00000 & -0.002 & -0.099 & -0.082 & -0.115 & 0.00000 & -0.002 & -0.116 & -0.066 \\
\hline
\end{tabular}


Table S9: Evaluation of the different contribution of the PFP model for the Eugenol(1) + $\mathrm{n}$-hexane(2) binary system at $283.15 \mathrm{~K}$ and comparison with experimental data.

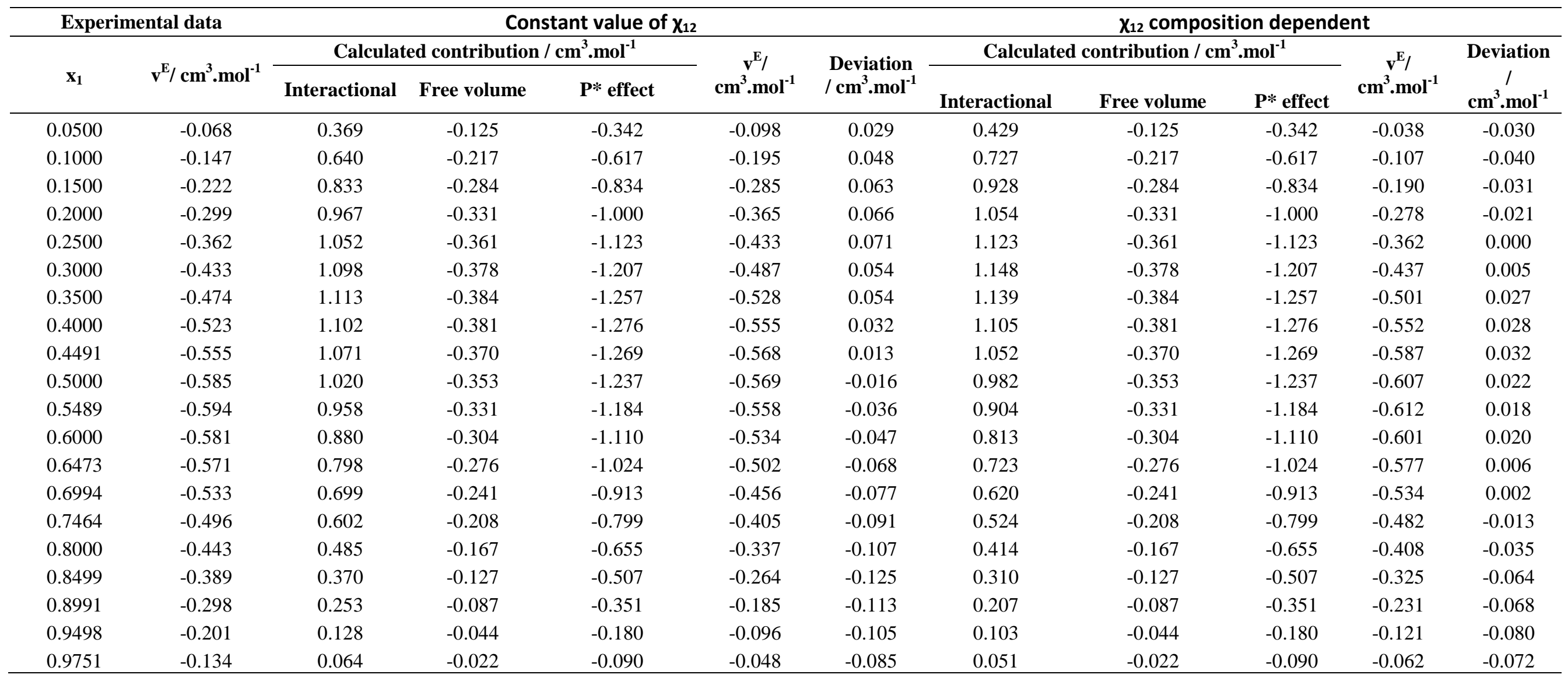




\section{References}

1. Rowley, R. L. "DIPPR ${ }^{\circledast}$ Data Compilation of Pure Chemical Properties." Design Institute for Physical Properties (2010).

2. Frenkel, M., Chirico, R. D., Diky, V., Yan, X., Dong, Q., Muzny, C.: ThermoData Engine (TDE): software implementation of the dynamic data evaluation concept. Journal of chemical information and modeling, 45, 816-838 (2005).

3. Aspen Plus. "Aspen Technology." Inc., version 9 (2016).

4. Guthrie Jr, G. B., Scott, D. W., Hubbard, W. N., Katz, C., McCullough, J. P., Gross, M. E., Williamson, K.D., Waddington, G.:Thermodynamic properties of furan, Journal of the American Chemical Society, 74, 4662-4669 (1952).

5. Timmermans, J., Hennaut-Roland, M.: Work of the International Bureau of Physico-Chemical Properties physical constants of twenty organic compounds, J. Chim. Phys. Phys.-Chim. Biol., 56, 9841023 (1959)

6. Bingham, E. C.; Spooner, L. W.: The Fluidity Method for the Determination of Association. I.J. Rheol. N. Y., 3, 221-244 (1932) 